\title{
Efficient Truncated Statistics with Unknown Truncation
}

\author{
Vasilis Kontonis \\ UW Madison \\ kontonis@wisc.edu
}

\author{
Christos Tzamos \\ UW Madison \\ tzamos@wisc.edu
}

\author{
Manolis Zampetakis \\ MIT \\ mzampet@mit.edu
}

August 6, 2019

\begin{abstract}
We study the problem of estimating the parameters of a Gaussian distribution when samples are only shown if they fall in some (unknown) subset $S \subseteq \mathbb{R}^{d}$. This core problem in truncated statistics has long history going back to Galton, Lee, Pearson and Fisher. Recent work by Daskalakis et al. (FOCS'18), provides the first efficient algorithm that works for arbitrary sets in high dimension when the set is known, but leaves as an open problem the more challenging and relevant case of unknown truncation set.

Our main result is a computationally and sample efficient algorithm for estimating the parameters of the Gaussian under arbitrary unknown truncation sets whose performance decays with a natural measure of complexity of the set, namely its Gaussian surface area. Notably, this algorithm works for large families of sets including intersections of halfspaces, polynomial threshold functions and general convex sets. We show that our algorithm closely captures the tradeoff between the complexity of the set and the number of samples needed to learn the parameters by exhibiting a set with small Gaussian surface area for which it is information theoretically impossible to learn the true Gaussian with few samples.
\end{abstract}




\section{Introduction}

A classical challenge in Statistics is estimation from truncated samples. Truncation occurs when samples falling outside of some subset $S$ of the support of the distribution are not observed. Truncation of samples has myriad manifestations in business, economics, engineering, social sciences, and all areas of the physical sciences.

Statistical estimation under truncated samples has had a long history in Statistics, going back to at least the work of Galton [Ga197] who analyzed truncated samples corresponding to speeds of American trotting horses. Following Galton's work, Pearson and Lee [Pea02, PL08, Lee14] used the method of moments in order to estimate the mean and standard deviation of a truncated univariate normal distribution and later Fisher [Fis31] used the maximum likelihood method for the same estimation problem. Since then, there has been a large volume of research devoted to estimating the truncated normal distribution; see e.g. [Sch86, Coh16, BC14]. Nevertheless, the first algorithm that is provably computationally and statistically efficient was only recently developed by Daskalakis et al. [DGTZ18], under the assumption that the truncation set $S$ is known.

In virtually all these works the question of estimation under unknown truncation set is raised. Our work resolves this question by providing tight sample complexity guarantees and an efficient algorithm for recovering the underlying Gaussian distribution. Although this estimation problem has clear and important practical and theoretical motivation too little was known prior to our work even in the asymptotic regime. In the early work of Shah and Jaiswal [SJ66] it was proven that the method of moments can be used to estimate a single dimensional Gaussian distribution when the truncation set is unknown but it is assumed to be an interval. In the other extreme where the set is allowed to be arbitrarily complex, Daskalakis et al. [DGTZ18] showed that it is information theoretically impossible to recover the parameters. We provide the first complete analysis of the number of samples needed for recovery taking into account the complexity of the underlying set.

Our Contributions. Our work studies the estimation task when the truncation set belongs in a family $\mathcal{C}$ of "low complexity". We use two different notions for quantifying the complexity of sets: the VC-dimension and the Gaussian Surface Area.

Our first result is that for any set family with $\mathrm{VC}$-dimension $\operatorname{VC}(\mathcal{C})$, the mean and covariance of the true $d$-dimensional Gaussian Distribution can be recovered up to accuracy $\varepsilon$ using only $\tilde{O}\left(\frac{\mathrm{VC}(\mathcal{C})}{\varepsilon}+\frac{d^{2}}{\varepsilon^{2}}\right)$ truncated samples.

Informal Theorem 1. Let $\mathcal{C}$ be a class of sets with $V C$-dimension $\operatorname{VC}(\mathcal{C})$ and let $N=\tilde{O}\left(\frac{\mathrm{VC}(\mathcal{C})}{\varepsilon}+\frac{d^{2}}{\varepsilon^{2}}\right)$. Given $N$ samples from a d-dimensional Gaussian $\mathcal{N}(\boldsymbol{\mu}, \boldsymbol{\Sigma})$ with unknown mean $\mu$ and covariance $\Sigma$, truncated on a set $S \in \mathcal{C}$ with mass at least $\alpha$, it is possible to find an estimate $(\hat{\mu}, \hat{\Sigma})$ such that $d_{\mathrm{TV}}(\mathcal{N}(\boldsymbol{\mu}, \boldsymbol{\Sigma}), \mathcal{N}(\hat{\boldsymbol{\mu}}, \hat{\Sigma})) \leq \varepsilon$

The estimation method computes the set of smallest mass that maximizes the likelihood of the data observed and learns the truncated distribution within error $O(\varepsilon)$ in total variation distance. To translate this error in total variation to parameter distance, we prove a general result showing that it is impossible to create a set (no matter the complexity) so that two Gaussians whose parameters are far have similar truncated distributions (see Lemma 3).

A simple but not successful approach would be to first try to learn an approximation of the truncation set with symmetric difference roughly $\varepsilon^{2} / d^{2}$ with the true set and then run the 
algorithm of [DGTZ18] using the approximate oracle. This approach would lead to a $\operatorname{VC}(\mathcal{S}) d^{2} / \varepsilon^{2}$ sample complexity that is worse than what we get. More importantly, doing empirical risk minimization $^{1}$ using truncated samples does not guarantee that we will find a set of small symmetric difference with the true and it is not clear how one could achieve that.

Our result bounds the sample complexity of identifying the underlying Gaussian distribution in terms of the VC-dimension of the set but does not yield a computationally efficient method for recovery. Obtaining a computationally efficient algorithm seems unlikely, unless one restricts attention to simple specific set families, such as axis aligned rectangles. One would hope that exploiting the fact that samples are drawn from a "tame" distribution, such as a Gaussian, can lead to general computationally efficient algorithms and even improved sample complexity.

Indeed, our main result is an algorithm that is both computationally and statistically efficient for estimating the parameters of a spherical Gaussian and uses only $d^{O\left(\Gamma^{2}(\mathcal{C})\right)}$ samples, where $\Gamma(\mathcal{C})$ is the Gaussian Surface Area of the class $\mathcal{C}$, an alternative complexity measure introduced by Klivans et al. [KOS08]:

Informal Theorem 2. Let $\mathcal{C}$ be a class of sets with Gaussian surface area at most $\Gamma(\mathcal{C})$ and let $k=$ poly $(1 / \alpha, 1 / \varepsilon) \Gamma(\mathcal{C})^{2}$. Given $N=d^{k}$ samples from a spherical d-dimensional Gaussian $\mathcal{N}\left(\boldsymbol{\mu}, \sigma^{2} \boldsymbol{I}\right)$, truncated on a set $S \in \mathcal{C}$ with mass at least $\alpha$, in time $\operatorname{poly}(m)$, we can find an estimate $\hat{\mu}, \hat{\sigma}^{2}$ such that

$$
d_{\mathrm{TV}}\left(\mathcal{N}\left(\boldsymbol{\mu}, \sigma^{2} \boldsymbol{I}\right), \mathcal{N}\left(\hat{\boldsymbol{\mu}}, \hat{\sigma}^{2} \boldsymbol{I}\right)\right) \leq \varepsilon .
$$

The notion of Gaussian surface area can lead to better sample complexity bounds even when the VC dimension is infinite. An example of such a case is when $\mathcal{C}$ is the class of all convex sets. Table 1 summarizes the known bounds for the Gaussian surface area of different concept classes and the implied sample complexity in our setting when combined with our main theorem.

\begin{tabular}{lcc}
\hline Concept Class & Gaussian Surface Area & Sample Complexity \\
\hline \hline Polynomial threshold functions of degree $k$ & $O(k)[\operatorname{Kan} 11]$ & $d^{O\left(k^{2}\right)}$ \\
Intersections of $k$ halfspaces & $O(\sqrt{\log k})[\mathrm{KOS} 08]$ & $d^{O(\log k)}$ \\
General convex sets & $O\left(d^{1 / 4}\right)[\mathrm{Ba} 193]$ & $d^{O(\sqrt{d})}$ \\
\hline \hline
\end{tabular}

Table 1: Summary of known results for Gaussian Surface Area. The last column gives the sample complexity we obtain for our setting.

Beyond spherical Gaussians, our main result extends to Gaussians with arbitrary diagonal covariance matrices. In addition, we provide an information theoretic result showing that the case with general covariance matrices can also be estimated using the same sample complexity bound by finding a Gaussian and a set that matches the moments of the true distribution. We remark our main algorithmic result Informal Theorem 3 uses Gaussian Surface Area whereas our sample complexity result Informal Theorem 2 uses VC-dimension. We discuss the differences of the two approaches in Section 7.

Informal Theorem 3. Let $\mathcal{C}$ be a class of sets with Gaussian surface area at most $\Gamma(\mathcal{C})$ and let $k=$ poly $(1 / \alpha, 1 / \varepsilon) \Gamma(\mathcal{C})^{2}$. Any truncated Gaussian with $\mathcal{N}(\hat{\mu}, \hat{\Sigma}, \hat{S})$ with $\hat{S} \in \mathcal{C}$ that approximately matches the moments up to degree $k$ of a truncated d-dimensional Gaussian $\mathcal{N}(\mu, \Sigma, S)$ with $S \in \mathcal{C}$, satisfies

\footnotetext{
${ }^{1}$ That is finding a set of the family that contains all the observed samples.
} 
$d_{\mathrm{TV}}(\mathcal{N}(\boldsymbol{\mu}, \boldsymbol{\Sigma}), \mathcal{N}(\hat{\boldsymbol{\mu}}, \hat{\boldsymbol{\Sigma}})) \leq \varepsilon$. The number of samples to estimate the moments within the required accuracy is at most $d^{O(k)}$.

This shows that the first few moments are sufficient to identify the parameters. Analyzing the guarantees of moment matching methods is notoriously challenging as it involves bounding the error of a system of many polynomial equations. Even for a single-dimensional Gaussian with truncation in an interval, where closed form solutions of the moments exist, it is highly non-trivial to bound these errors [SJ66]. In contrast, our analysis using Hermite polynomials allows us to easily obtain bounds for arbitrary truncation sets in high dimensions, even though no closed form expression for the moments exists.

We conclude by showing that the dependence of our sample complexity bounds both on the VC-dimension and the Gaussian Surface Area is tight up to polynomial factors. In particular, we construct a family in $d$ dimensions with VC dimension $2^{d}$ and Gaussian surface area $O(d)$ for which it is not possible to learn the mean of the underlying Gaussian within 1 standard deviation using $o\left(2^{d / 2}\right)$ samples.

Informal Theorem 4. There exists a family of sets $\mathcal{S}$ with $\Gamma(\mathcal{S})=O(d)$ and VC-dimension $2^{d}$ such that any algorithm that draws $N$ samples from $\mathcal{N}(\boldsymbol{\mu}, \boldsymbol{I}, S)$ and computes an estimate $\tilde{\boldsymbol{\mu}}$ with $\|\widetilde{\boldsymbol{\mu}}-\boldsymbol{\mu}\|_{2} \leq 1$ must have $N=\Omega\left(2^{d / 2}\right)$.

Our techniques and relation to prior work. The work of Klivans et al. [KOS08] provides a computationally and sample efficient algorithm for learning geometric concepts from labeled examples drawn from a Gaussian distribution. On the other hand, the recent work of Daskalakis et al. [DGTZ18] provides efficient estimators for truncated statistics with known sets. One could hope to combine these two approaches for our setting, by first learning the set and then using the algorithm of [DGTZ18] to learn the parameters of the Gaussian. This approach, however, fails for two reasons. First, the results of Klivans et al. [KOS08] apply in the supervised learning setting where one has access to both positive and negative samples, while our problem can be thought of as observing only positive examples (those falling inside the set). In addition, any direct approach that extends their result to work with positive only examples requires that the underlying Gaussian distribution is known in advance.

One of our key technical contributions is to extend the techniques of Klivans et al. [KOS08] to work with positive only examples from an unknown Gaussian distribution, which is the major case of interest in truncated statistics. To perform the set estimation Klivans et al. [KOS08], rely on a family of orthogonal polynomials with respect to the Gaussian distribution, namely the Hermite polynomials and show that the indicator function of the set is well approximated by its low degree Hermite expansion. While we cannot learn this function directly in our setting, we are able to recover an alternative function, that contains "entangled" information of both the true Gaussian parameters and the underlying set. After learning the function, we formulate an optimization problem whose solution enables us to decouple these two quantities and retrieve both the Gaussian parameters and the underlying set. We describe our estimation method in more detail in Section 4. As a corollary of our approach, we obtain the first efficient algorithm for learning geometric concepts from positive examples drawn from an unknown spherical Gaussian distribution. 


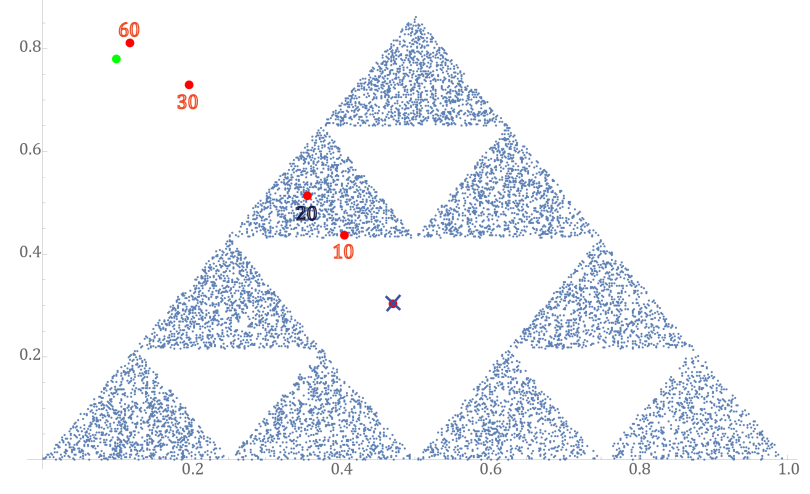

(a) Execution of our algorithm for isotropic Gaussian distribution with $\boldsymbol{\mu}^{*}=(0.1,0.78)$ and $\mu_{S}=$ $(0.48,0.32)$.

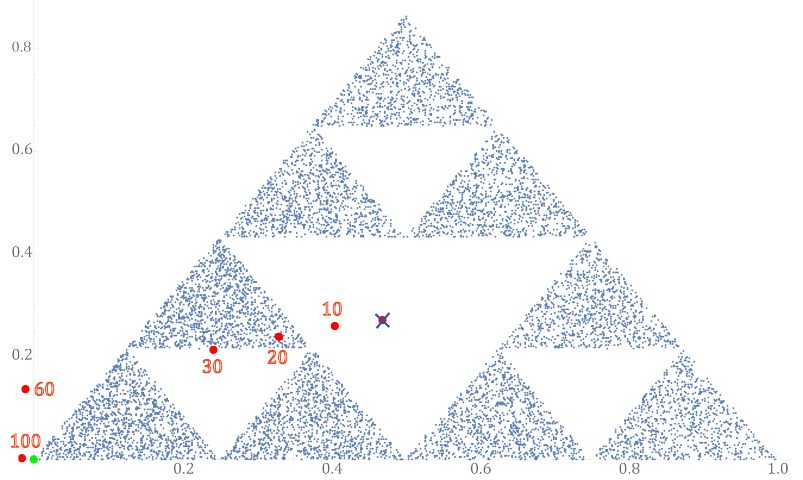

(b) Execution of our algorithm for isotropic Gaussian distribution with $\boldsymbol{\mu}^{*}=(0,0)$ and $\boldsymbol{\mu}_{\mathrm{S}}=$ $(0.47,0.27)$.

Figure 1: Illustration of the results of our algorithm for an unknown truncation set. The $x$ sign corresponds to the conditional mean of the truncated distribution, while the green point corresponds to the true mean and the red points correspond to the estimated true mean depending on the degree of the Hermite polynomials that are being used by the algorithm.

Simulations. In addition to the theoretical guarantees of our algorithm, we empirically evaluate its performance using simulated data. We present the results that we get in Figure 1, where one can see that even when the truncation set is complex, our algorithm finds an accurate estimation of the mean of the untruncated distribution. Observe that our algorithm succeeds in estimating the true mean of the input distribution despite the fact that the set is unknown and the samples look similar in both cases.

\subsection{Further Related Work}

Our work is related to the field of robust statistics as it can robustly learn a Gaussian even in the presence of an adversary erasing samples outside a certain set. Recently, there has been a lot of theoretical work doing robust estimation of the parameters of multi-variate Gaussian distributions in the presence of arbitrary corruptions to a small $\varepsilon$ fraction of the samples, allowing for both deletions of samples and additions of samples that can also be chosen adaptively $\left[\mathrm{DKK}^{+} 16, \mathrm{CSV} 17, \mathrm{LRV}^{16}, \mathrm{DKK}^{+} 17, \mathrm{DKK}^{+} 18\right]$. When the corruption of the data is so powerful it is easy to see that the estimation error of the parameter depends on $\varepsilon$ and cannot shrink to 0 as the number of samples grows to infinity. In our model the corruption is more restrictive but in return our results show how to estimate the parameters of a multi-variate Gaussian distribution to arbitrary accuracy even when the fraction of corruption is any constant less than 1.

Our work also has connections with the literature of learning from positive examples. At the heart of virtually all of the results in this literature is the use of the exact knowledge of the original non-truncated distribution to be able to generate fake negative examples, e.g. [Den98, LDG00]. When the original distribution is uniform, better algorithms are known. Diakonikolas et al. [DDS14] gave efficient learning algorithms for DNFs and linear threshold functions, Frieze et al. [FJK96] and Anderson et al. [AGR13] gave efficient learning algorithms for learning $d$-dimensional simplices. Another line of work proves lower bounds on the sample complexity of recovering an 
unknown set from positive examples. Goyal et al. [GR09] showed that learning a convex set in $d$-dimensions to accuracy $\varepsilon$ from positive samples, uniformly distributed inside the set, requires at least $2^{\Omega(\sqrt{d / \varepsilon})}$ samples, while the work of [Eld11] showed that $2^{\Omega(\sqrt{d})}$ samples are necessary even to estimate the mass of the set. To the best of our knowledge, no matching upper bounds are known for those results. Our estimation result implies that $d^{\text {poly }\left(\frac{1}{\varepsilon}\right) \sqrt{d}}$ are sufficient to learn the set and its mass when given positive samples from a Gaussian truncated on the convex set.

\section{Preliminaries}

Notation. We use small bold letters $x$ to refer to real vectors in finite dimension $\mathbb{R}^{d}$ and capital bold letters $A$ to refer to matrices in $\mathbb{R}^{d \times \ell}$. Similarly, a function with image in $\mathbb{R}^{d}$ is represented by a small and bold letter $f$. Given a subset $S$ of $\mathbb{R}^{d}$ we define $\mathbf{1}_{S}(x)$ to be its $0-1$ indicator. Let $A \in \mathbb{R}^{d \times d}$, we define $A^{b} \in \mathbb{R}^{d^{2}}$ to be the standard vectorization of $A$. Let also $\mathcal{Q}_{d}$ be the set of all the symmetric $d \times d$ matrices. The Frobenius norm of a matrix $A$ is defined as $\|A\|_{F}=\left\|A^{b}\right\|_{2}$.

Gaussian Distribution. Let $\mathcal{N}(\boldsymbol{\mu}, \boldsymbol{\Sigma})$ be the normal distribution with mean $\boldsymbol{\mu}$ and covariance matrix $\Sigma$, with the following probability density function

$$
\mathcal{N}(\boldsymbol{\mu}, \boldsymbol{\Sigma} ; \boldsymbol{x})=\frac{1}{\sqrt{\operatorname{det}(2 \pi \Sigma)}} \exp \left(-\frac{1}{2}(\boldsymbol{x}-\boldsymbol{\mu})^{T} \boldsymbol{\Sigma}^{-1}(\boldsymbol{x}-\boldsymbol{\mu})\right) .
$$

Also, let $\mathcal{N}(\mu, \Sigma ; S)$ denote the probability mass of a measurable set $S$ under this Gaussian measure. We shall also denote by $\mathcal{N}_{0}$ the standard Gaussian, whether it is single or multidimensional will be clear from the context.

Truncated Gaussian Distribution. Let $S \subseteq \mathbb{R}^{d}$ be a subset of the $d$-dimensional Euclidean space, we define the $S$-truncated normal distribution $\mathcal{N}(\mu, \Sigma, S)$ the normal distribution $\mathcal{N}(\boldsymbol{\mu}, \boldsymbol{\Sigma})$ conditioned on taking values in the subset $S$. The probability density function of $\mathcal{N}(\mu, \Sigma, S)$ is the following

$$
\mathcal{N}(\mu, \Sigma, S ; x)=\frac{\mathbf{1}_{S}(x)}{\mathcal{N}(\mu, \Sigma ; S)} \mathcal{N}(\mu, \Sigma ; x)
$$

We will assume that the covariance matrix $\Sigma$ is full rank. The case where $\Sigma$ is not full rank we can easily detect and solve the estimation problem in the linear subspace of samples.

The core complexity measure of Borel sets in $\mathbb{R}^{d}$ that we use is the notion of Gaussian Surface Area defined below.

Definition 1 (Gaussian Surface Area). For a Borel set $A \subseteq \mathbb{R}^{d}, \delta \geq 0$ let $A_{\delta}=\{x: \operatorname{dist}(x, A) \leq \delta\}$. The Gaussian surface area of $A$ is

$$
\Gamma(A)=\liminf _{\delta \rightarrow 0} \frac{\mathcal{N}_{0}\left(A_{\delta} \backslash A\right)}{\delta} .
$$

We define the Gaussian surface area of a family of sets $\mathcal{C}$ to be $\Gamma(\mathcal{C})=\sup _{C \in \mathcal{C}} \Gamma(C)$.

\subsection{Problem formulation}

Given samples from a truncated Gaussian $\mathcal{N}_{S}^{*} \triangleq \mathcal{N}\left(\mu^{*}, \Sigma^{*}, S\right)$, our goal is to learn the parameters $\left(\boldsymbol{\mu}^{*}, \boldsymbol{\Sigma}^{*}\right)$ and recover the set $S$. We denote by $\alpha^{*}=\mathcal{N}\left(\boldsymbol{\mu}^{*}, \boldsymbol{\Sigma}^{*} ; S\right)$, the total mass contained in set $S$ 
by the untruncated Gaussian $\mathcal{N}^{*} \triangleq \mathcal{N}\left(\boldsymbol{\mu}^{*}, \boldsymbol{\Sigma}^{*}\right)$. Throughout this paper, we assume that we know an absolute constant $\alpha>0$ such that

$$
\mathcal{N}\left(\mu^{*}, \Sigma^{*} ; S\right)=\alpha^{*} \geq \alpha
$$

\section{Identifiability with bounded VC dimension}

In this section we analyze the sample compexity of learning the true Gaussian parameters when the truncation set has bounded VC-dimension. In particular we show that the overhead over the $d^{2} / \varepsilon^{2}$ samples (which is the sample compexity of learning the parameters of the Gaussian without truncation) is proportional to the VC dimension of the class.

Theorem 1. Let $\mathcal{S}$ be a family of sets of finite $V C$ dimension, and let $\mathcal{N}(\mu, \Sigma, S)$ be a truncated Gaussian distribution such that $\mathcal{N}(\boldsymbol{\mu}, \boldsymbol{\Sigma} ; S) \geq \alpha$. Given $N$ samples with

$$
N=\operatorname{poly}(1 / \alpha) \widetilde{O}\left(\frac{d^{2}}{\varepsilon^{2}}+\frac{\operatorname{VC}(\mathcal{S})}{\varepsilon}\right)
$$

Then, with probability at least $99 \%$, it is possible to identify $(\widetilde{\boldsymbol{\mu}}, \widetilde{\boldsymbol{\Sigma}})$ that satisfy $d_{\mathrm{TV}}(\mathcal{N}(\boldsymbol{\mu}, \boldsymbol{\Sigma}), \mathcal{N}(\widetilde{\boldsymbol{\mu}}, \widetilde{\boldsymbol{\Sigma}})) \leq \varepsilon$ and $\left\|\boldsymbol{\Sigma}^{-1 / 2}(\boldsymbol{\mu}-\widetilde{\boldsymbol{\mu}})\right\|_{2} \leq \varepsilon$ and $\left\|\boldsymbol{I}-\boldsymbol{\Sigma}^{-1 / 2} \widetilde{\boldsymbol{\Sigma}} \boldsymbol{\Sigma}^{-1 / 2}\right\|_{F} \leq \varepsilon$.

Our algorithm works by first learning the truncated distribution within total variation distance $\varepsilon$. To do this, we first assume that we know the mean and covariance of the underlying Gaussian by guessing the parameters and accurately learn the underlying set. After drawing $N=\Theta\left(\frac{\mathrm{VC}(\mathcal{S}) \log (1 / \varepsilon)}{\varepsilon}\right)$ samples from the distribution, any set in the class that contains the samples will only exclude at most an $\varepsilon$ fraction of the total mass. Picking the set $\widetilde{S}$ that maximizes the likelihood of those samples, i.e. the set with minimum mass according to the guessed Gaussian distribution, guarantees that the total variation distance between the learned truncated distribution and the true is at most $\varepsilon$, if the guess of the parameters was accurate (Lemma 1). The proof of Lemma 1 can be found in Appendix B.

Lemma 1. Let $\mathcal{S}$ be a family of subsets in $\mathbb{R}^{d}$ and Let $\mathcal{N}\left(\mu, \Sigma, S^{*}\right)=\mathcal{N}_{S}^{*}$ be a Normal distribution truncated on the set $S^{*} \in \mathcal{S}$. Fix $\varepsilon \in(0,1), \delta \in(0,1 / 4)$ and let

$$
N=O\left(\frac{\operatorname{VC}(\mathcal{S}) \log (1 / \varepsilon)}{\varepsilon}+\log \left(\frac{1}{\delta}\right)\right)
$$

Moreover, let $\widetilde{\boldsymbol{\mu}}, \widetilde{\boldsymbol{\Sigma}}$ be such that $d_{\mathrm{TV}}(\mathcal{N}(\widetilde{\boldsymbol{\mu}}, \widetilde{\boldsymbol{\Sigma}}), \mathcal{N}(\boldsymbol{\mu}, \boldsymbol{\Sigma})) \leq \varepsilon$. Assume that we draw $N$ samples $\boldsymbol{x}_{i}$ from $\mathcal{N}_{S^{*}}$, Let $\widetilde{S}$ be the solution of the problem

$$
\min _{S} \mathcal{N}(\widetilde{\boldsymbol{\mu}}, \widetilde{\Sigma} ; S) \text { subject to } x_{i} \in S \text { for all } i \in[n]
$$

Then with probability at least $1-\delta$ we have $d_{\mathrm{TV}}(\mathcal{N}(\widetilde{\mu}, \widetilde{\Sigma}, \widetilde{S}), \mathcal{N}(\boldsymbol{\mu}, \boldsymbol{\Sigma}, S)) \leq 3 \varepsilon /(2 \alpha)$.

This is because the total variation distance between two densities $f$ and $g$ can be written as $\int(f(x)-g(x)) \mathbf{1}_{f(x)>g(x)} d x$. Note that by choosing the set of the smallest mass consistent with the samples, we guarantee that the guess will have higher density at every point apart from those 
outside the support $\widetilde{S}$. However, as we argued the outside mass is at most $\varepsilon$ with respect to the true distribution which gives the bound in the total variation distance.

To remove the assumption that the true parameters are known, we build a cover of all possible mean and covariance matrices that the underlying Gaussian might have and run the tournament from [DK14] to identify the best one (Lemma 20). While there are $(d / \varepsilon)^{O\left(d^{2}\right)}$ such parameters, the number of samples needed for running the tournament is only logarithmic which shows that an additional $\widetilde{O}\left(d^{2} / \varepsilon^{2}\right)$ are sufficient to find a hypothesis in total variation distance $\varepsilon$ (Lemma 2). The proof of Lemma 2 can be found in Appendix B.

Lemma 2. Let $S \in \mathcal{S}$ be a subset of $\mathbb{R}^{d}$ and $\mathcal{N}(\mu, \Sigma, S)$ be the corresponding truncated normal distribution. Then $\widetilde{O}\left(\operatorname{VC}(\mathcal{S}) / \varepsilon+d^{2} / \varepsilon^{2}\right)$ samples are sufficient to find parameters $\widetilde{\mu}, \widetilde{\Sigma}, \widetilde{S}$ such that $d_{\mathrm{TV}}(\mathcal{N}(\mu, \Sigma, S), \mathcal{N}(\widetilde{\mu}, \widetilde{\Sigma}, \widetilde{S})) \leq \varepsilon$ with probability at least $99 \%$.

We finally argue that the $\varepsilon$ error in total variation of the truncated distributions translates to an $O(\varepsilon)$ bound in total variation distance of the untruncated distributions (Lemma 3). We show that this is true in general and does not depend on the complexity of the set. To prove this statement, we consider two Gaussians with parameters that are far from each other and construct the worst possible set to make their truncated distributions as close as possible. We show that under the requirement that the set contains at least $\alpha$ mass, the total variation distance of the truncated distributions will be large.

Lemma 3 (Total Variation of Truncated Normals). Let $D_{1}=\mathcal{N}\left(\boldsymbol{\mu}_{1}, \boldsymbol{\Sigma}_{1}, S_{1}\right)$ and $D_{2}=\mathcal{N}\left(\boldsymbol{\mu}_{2}, \boldsymbol{\Sigma}_{2}, S_{2}\right)$ be two truncated Normal distributions such that $\mathcal{N}\left(\boldsymbol{\mu}_{1}, \boldsymbol{\Sigma}_{1} ; S_{1}\right), \mathcal{N}\left(\boldsymbol{\mu}_{2}, \boldsymbol{\Sigma}_{2} ; S_{2}\right) \geq \alpha$. Then

$$
d_{\mathrm{TV}}\left(D_{1}, D_{2}\right) \geq C_{\alpha} d_{\mathrm{TV}}\left(\mathcal{N}\left(\boldsymbol{\mu}_{1}, \boldsymbol{\Sigma}_{1}\right), \mathcal{N}\left(\boldsymbol{\mu}_{2}, \boldsymbol{\Sigma}_{2}\right)\right)
$$

where $C_{\alpha}<\alpha / 8$ is a positive constant that only depends on $\alpha, C_{\alpha}=\Omega\left(\alpha^{3}\right)$.

Proof. Without loss of generality we assume that $D_{1}=\mathcal{N}\left(\mathbf{0}, I, S_{1}\right)$ and $D_{2}=\mathcal{N}\left(\mu, \Lambda, S_{2}\right)$, where $\Lambda$ is a diagonal matrix. We want to find the worst sets $S_{1}, S_{2}$ so that $d_{\mathrm{TV}}\left(D_{1}, D_{2}\right)$ is small. If $D_{1}\left(S_{1} \backslash S_{2}\right) \geq \alpha / 2$ then the statement holds. Therefore, we consider the set $S=S_{1} \cap S_{2}$ and relax the constraint that the truncated Gaussian $D_{2}$ integrates to 1 . Taking into account the fact that the set $S=S_{1} \cap S_{2}$ must have at least some mass $\alpha / 2$ with respect to $\mathcal{N}(\mathbf{0}, \boldsymbol{I})$, the following optimization problem provides a lower bound on the total variation distance of $D_{1}$ and $D_{2}$.

$$
\begin{array}{ll}
\min _{S \in \mathcal{S}, \beta>0} & \frac{1}{\alpha} \int\left|\mathcal{N}(\mathbf{0}, \boldsymbol{I} ; \boldsymbol{x})-\frac{\alpha}{\beta} \mathcal{N}(\boldsymbol{\mu}, \boldsymbol{\Lambda} ; \boldsymbol{x})\right| \mathbf{1}_{S}(\boldsymbol{x}) \mathrm{d} x \\
\text { subj. to } & \int \mathcal{N}(\mathbf{0}, \boldsymbol{I} ; \boldsymbol{x}) \mathbf{1}_{S}(\boldsymbol{x}) \mathrm{d} \boldsymbol{x} \geq \alpha / 2,
\end{array}
$$

For any fixed $\beta>0$ this is a fractional knapsack problem and therefore we should include in the set the points $x$ in order of increasing ratio of weight that is contribution to the $L_{1}$ error $\left|\mathcal{N}(\mathbf{0}, \mathbf{I} ; \boldsymbol{x})-\frac{\alpha}{\beta} \mathcal{N}(\boldsymbol{\mu}, \boldsymbol{\Lambda} ; \boldsymbol{x})\right|$, over value, that is density $\mathcal{N}(\mathbf{0}, \mathbf{I} ; \boldsymbol{x})$ until we reach some threshold $T$. Therefore, the set is defined to be

$$
S=\left\{\boldsymbol{x} \in \mathbb{R}^{d}: \frac{\left|\mathcal{N}(\mathbf{0}, \boldsymbol{I} ; \boldsymbol{x})-\frac{\alpha}{\beta} \mathcal{N}(\boldsymbol{\mu}, \boldsymbol{\Lambda} ; \boldsymbol{x})\right|}{\mathcal{N}(\mathbf{0}, \mathbf{I} ; \boldsymbol{x})} \leq T\right\}=\left\{\boldsymbol{x} \in \mathbb{R}^{d}:|1-\exp (p(\boldsymbol{x}))| \leq T\right\}
$$


where $p(x)=-\frac{1}{2}(\boldsymbol{\mu}-\boldsymbol{x})^{T} \boldsymbol{\Lambda}^{-1}(\boldsymbol{\mu}-\boldsymbol{x})+\frac{1}{2} \boldsymbol{x}^{T} \boldsymbol{x}+\log (\alpha /(\sqrt{|\boldsymbol{\Lambda}|} \beta))$. Using Theorem 9 for the degree 2 polynomial $p(\boldsymbol{x})$ and setting $q=4, \gamma=\alpha^{2}\left(\mathbb{E}_{x \sim \mathcal{N}_{0}} p^{2}(x)\right)^{1 / 2} /\left(256 C^{2}\right)$, where $C$ is the absolute constant of Theorem 9, we get that

$$
\mathcal{N}_{0}(\{z:|p(z)| \leq \gamma\}) \leq \frac{\alpha}{4}
$$

To simplify notation set $Q=\{z:|p(z)| \leq \gamma\}$. Therefore, for any $x$ in the remaining $\alpha / 4$ mass of the set $S$ we know that $|p(\boldsymbol{x})| \geq \gamma$. Next, we lower bound $\gamma$ in terms of the distance of the parameters of the two Gaussians. We have

$$
\begin{aligned}
& \underset{x \sim \mathcal{N}_{0}}{\mathbb{E}}\left[p^{2}(\boldsymbol{x})\right] \geq \underset{\boldsymbol{x} \sim \mathcal{N}_{0}}{\operatorname{Var}}[p(\boldsymbol{x})]=\underset{x \sim \mathcal{N}_{0}}{\operatorname{Var}}\left[-\frac{1}{2}(\boldsymbol{\mu}-\boldsymbol{x})^{T} \boldsymbol{\Lambda}^{-1}(\boldsymbol{\mu}-\boldsymbol{x})+\frac{1}{2} \boldsymbol{x}^{T} \boldsymbol{x}\right] \\
& =\operatorname{Var}_{x \sim \mathcal{N}_{0}}\left[\sum_{i=1}^{d}\left(\frac{\mu_{i}}{\lambda_{i}} x+x^{2} \frac{\left(1-1 / \lambda_{i}\right)}{2}\right)\right]=\sum_{i=1}^{d} \underset{x \sim \mathcal{N}(0,1)}{\operatorname{Var}}\left[\frac{\mu_{i}}{\lambda_{i}} x+x^{2} \frac{\left(1-1 / \lambda_{i}\right)}{2}\right] \\
& =\sum_{i=1}^{d} \frac{1}{2}\left(\frac{1}{\lambda_{i}}-1\right)^{2}+\frac{\mu_{i}^{2}}{\lambda_{i}^{2}}=\frac{1}{2}\left\|\boldsymbol{\Lambda}^{-1}-\boldsymbol{I}\right\|_{F}^{2}+\left\|\boldsymbol{\Lambda}^{-1 / 2} \boldsymbol{\mu}\right\|_{2}^{2}
\end{aligned}
$$

Therefore, using the inequality $\sqrt{2} \sqrt{x+y} \geq \sqrt{x}+\sqrt{y}$ we obtain

$$
\gamma \geq \frac{\alpha^{2}}{256 \sqrt{2} C^{2}}\left(\frac{1}{\sqrt{2}}\left\|\Lambda^{-1}-\boldsymbol{I}\right\|_{F}+\left\|\Lambda^{-1 / 2} \boldsymbol{\mu}\right\|_{2}\right) \geq \frac{\alpha^{2}}{256 C^{2}} d_{\mathrm{TV}}\left(\mathcal{N}\left(\boldsymbol{\mu}_{1}, \boldsymbol{\Sigma}_{1}\right), \mathcal{N}\left(\boldsymbol{\mu}_{2}, \boldsymbol{\Sigma}_{2}\right)\right)
$$

where we used Lemma 16. Assume first that $\gamma \leq 1$. We have that the $L_{1}$ distance between the functions $f(\boldsymbol{x})=\mathcal{N}(\mathbf{0}, \boldsymbol{I} ; \boldsymbol{x}) \mathbf{1}_{S}(\boldsymbol{x})$ and $g(\boldsymbol{x})=\frac{\alpha}{\beta} \mathcal{N}(\boldsymbol{\mu}, \boldsymbol{\Lambda} ; \boldsymbol{x}) \mathbf{1}_{S}(\boldsymbol{x})$ is

$$
\begin{aligned}
\int|f(x)-g(\boldsymbol{x})| \mathrm{d} x & =\underset{x \sim \mathcal{N}_{0}}{\mathbb{E}}\left[|1-\exp (p(\boldsymbol{x}))| \mathbf{1}_{S}(\boldsymbol{x})\right] \geq \underset{x \sim \mathcal{N}_{0}}{\mathbb{E}}\left[\frac{|p(x)|}{2} \mathbf{1}_{S \backslash Q}(\boldsymbol{x})\right] \\
& \geq \gamma \underset{x \sim \mathcal{N}_{0}}{\mathbb{E}}\left[\mathbf{1}_{S \backslash Q}(\boldsymbol{x})\right] \geq \frac{\alpha \gamma}{4} \geq C_{\alpha} d_{\mathrm{TV}}\left(\mathcal{N}\left(\boldsymbol{\mu}_{1}, \boldsymbol{\Sigma}_{1}\right), \mathcal{N}\left(\boldsymbol{\mu}_{2}, \boldsymbol{\Sigma}_{2}\right)\right),
\end{aligned}
$$

where for the first inequality we used the inequality $\left|1-\mathrm{e}^{x}\right| \geq|x| / 2$ for $|x| \leq 1$. Note that $C_{a}=\Omega\left(\alpha^{3}\right)$. If $\gamma>1$ we have

$$
\int|f(x)-g(x)| \mathrm{d} x=\underset{x \sim \mathcal{N}_{0}}{\mathbb{E}}\left[|1-\exp (p(x))| \mathbf{1}_{S}(\boldsymbol{x})\right] \geq \underset{x \sim \mathcal{N}_{0}}{\mathbb{E}}\left[\frac{1}{2} \mathbf{1}_{S \backslash Q}(\boldsymbol{x})\right] \geq \alpha / 8,
$$

where we used the inequality $\left|1-\mathrm{e}^{x}\right| \geq 1 / 2$ for $|x|>1$.

\section{Estimation Algorithm for bounded Gaussian Surface Area}

In this section, we present the main steps of our estimation algorithm. In later sections, we provide details of the individual components. The algorithm can be thought of in 3 stages.

First Stage In the first stage, our goal is to learn a weighted characteristic function of the underlying set. Even though we cannot access the underlying set directly, for any given function $f$ we can evaluate the expectation $\mathbb{E}_{x \sim \mathcal{N}\left(\mu^{*}, \Sigma^{*}, S\right)}[f(x)]$ using truncated samples. 
This expectation can be equivalently written as $\mathbb{E}_{\boldsymbol{x} \sim \mathcal{N}(\mathbf{0}, \boldsymbol{I})}[f(\boldsymbol{x}) \psi(\boldsymbol{x})]$ for the function

$$
\psi(\boldsymbol{x}) \triangleq \frac{\mathbf{1}_{S}(\boldsymbol{x})}{\alpha^{*}} \frac{\mathcal{N}\left(\boldsymbol{\mu}^{*}, \boldsymbol{\Sigma}^{*} ; \boldsymbol{x}\right)}{\mathcal{N}(\mathbf{0}, \boldsymbol{I} ; \boldsymbol{x})}=\frac{\mathbf{1}_{S}(\boldsymbol{x})}{\alpha^{*}} \frac{\mathcal{N}^{*}(\boldsymbol{x})}{\mathcal{N}_{0}(\boldsymbol{x})} .
$$

By evaluating the above expectation for different functions $f$ corresponding to the Hermite polynomials $H_{V}(\boldsymbol{x})$, we can recover $\psi(\boldsymbol{x})$, through its Hermite expansion:

$$
\psi(\boldsymbol{x})=\sum_{V \in \mathbb{N}^{d}} \underset{x \sim \mathcal{N}_{0}}{\mathbb{E}}\left[H_{V}(\boldsymbol{x}) \psi(\boldsymbol{x})\right] H_{V}(\boldsymbol{x})=\sum_{V \in \mathbb{N}^{d}} \underset{\boldsymbol{x} \sim \mathcal{N}_{S}^{*}}{\mathbb{E}}\left[H_{V}(\boldsymbol{x})\right] H_{V}(\boldsymbol{x}) .
$$

Of course, it is infeasible to calculate the Hermite expansion for any $V \in \mathbb{N}^{d}$. In Section 4.1, we show that by estimating only terms of degree at most $k$, we can achieve a good approximation to $\psi$ where the error depends on the Gaussian surface area of the underlying set $S$. To do this, we show that most of the mass of the coefficients $c_{V}=\mathbb{E}_{\boldsymbol{x} \sim \mathcal{N}_{0}}\left[H_{V}(\boldsymbol{x}) \psi(\boldsymbol{x})\right]$ is concentrated on low degree terms, i.e. $\sum_{|V|>k} c_{V}^{2}$ is significantly small. Moreover, we show that even though we can only estimate the coefficients $c_{V}$ through sampling, the sampling error is significantly small.

Overall, after the first stage, we obtain a non-negative function $\psi_{k}$ that is close to $\psi$. The approximation error guarantees are given in Theorem 4.

Second Stage Given the function $\psi_{k}$ that was recovered in the first stage, our goal is to decouple the influence of the set $\frac{1_{S}(x)}{\alpha^{*}}$ and the influence of the underlying Gaussian distribution which corresponds to the multiplicative term $\frac{\mathcal{N}\left(\mu^{*}, \Sigma^{*} ; x\right)}{\mathcal{N}(0, I ; x)}$. This would be easy if we had the exact function $\psi$ in hand. In contrast, for the polynomial function $\psi_{k}$ the problem is significantly challenging as it is only close to $\psi$ on average but not pointwise.

To perform the decoupling and identify the underlying Gaussian we explicitly multiply the function $\psi_{k}$ with a corrective term of the form $\frac{\mathcal{N}(0, I ; x)}{\mathcal{N}(\mu, \Sigma ; x)}$. We set up an optimization problem seeking to minimize the function $C(\boldsymbol{\mu}, \boldsymbol{\Sigma}) \mathbb{E}_{\boldsymbol{x} \sim \mathcal{N}_{S}^{*}}\left[\frac{\mathcal{N}(\mathbf{0}, \mathbf{I} ; \boldsymbol{x})}{\mathcal{N}(\boldsymbol{\mu}, \boldsymbol{\Sigma} ; \boldsymbol{x})} \psi_{k}(\boldsymbol{x})\right]$ with an appropriate choice of $C(\boldsymbol{\mu}, \boldsymbol{\Sigma})$ so that the unique solution corresponds to $(\boldsymbol{\mu}, \boldsymbol{\Sigma})=\left(\boldsymbol{\mu}^{*}, \boldsymbol{\Sigma}^{*}\right)$. Under a reparameterization of $(\boldsymbol{u}, \boldsymbol{B})=\left(\boldsymbol{\Sigma}^{-1} \boldsymbol{\mu}, \boldsymbol{\Sigma}^{-1}\right)$, we show that the corresponding problem is strongly convex. Still, optimizing it directly is non-trivial as it involves taking the expectation with respect to the unknown truncated Gaussian. Instead, we perform stochastic gradient descent (SGD) and show that it quickly converges in few steps to point close to the true minimizer (Algorithm 1).

This allows us to recover parameters $(\hat{\mu}, \hat{\Sigma})$ so that the total variation distance between the recovered and the true (untruncated) Gaussian is very small, i.e. $d_{T V}\left(\mathcal{N}(\hat{\mu}, \hat{\Sigma}), \mathcal{N}\left(\boldsymbol{\mu}^{*}, \boldsymbol{\Sigma}^{*}\right)\right) \leq \varepsilon$. Theorem 2 describes the guarantees of the second stage. Further details are provided in Section 4.2.

Third Stage Given the weighted indicator function $\psi_{k}$ and the recovered Gaussian $\mathcal{N}(\hat{\mu}, \hat{\Sigma})$, we move on to recover the underlying set $S$. To do this, we compute the function $\frac{\mathcal{N}(\mathbf{0}, \mathbf{I} ; \boldsymbol{x})}{\mathcal{N}(\hat{\mu}, \hat{\Sigma} ; \boldsymbol{x})} \psi_{k}(\boldsymbol{x})$ and set a threshold at $1 / 2$. It is easy to check that if there were no errors, i.e. $\psi_{k}=\psi$ and $d_{T V}\left(\mathcal{N}(\hat{\boldsymbol{\mu}}, \hat{\boldsymbol{\Sigma}}), \mathcal{N}\left(\boldsymbol{\mu}^{*}, \boldsymbol{\Sigma}^{*}\right)\right)=0$, that this thresholding step would correctly identify the set. In Section 4.3 we bound the error guarantees of this approach. We show that it is possible to obtain an estimate $\hat{S}$ of the underlying set so that the mass of the symmetric difference with the true Gaussian is small, i.e. $\mathcal{N}\left(\boldsymbol{\mu}^{*}, \boldsymbol{\Sigma}^{*} ; S \triangle \hat{S}\right)<\varepsilon$. Overall, our algorithm requires at most $d^{\text {poly }(1 / \alpha, 1 / \varepsilon) \Gamma^{2}(S)}$, where $\Gamma(S)$ is the Gaussian surface area of the set $S$ and $\alpha$ is a lower-bound on the mass that is assigned by the true Gaussian on the set $S$. The running time of our algorithm is linear in the number of samples. 
The guarantees of the algorithm We first show our algorithmic results under the assumption that the untruncated Gaussian $\mathcal{N}^{*}$ is known to be in near-isotropic position.

Definition 2 (Near-Isotropic Position). Let $\mu \in \mathbb{R}^{d}, \Sigma \in \mathbb{R}^{d \times d}$ be a positive semidefinite symmetric matrix and $a, b>0$. We say that $(\boldsymbol{\mu}, \boldsymbol{\Sigma})$ is in $(a, b)$-isotropic position if the following hold.

$$
\|\boldsymbol{\mu}\|_{2}^{2} \leq a, \quad\|\boldsymbol{\Sigma}-\boldsymbol{I}\|_{F}^{2} \leq a, \quad(1-b) \boldsymbol{I} \preceq \boldsymbol{\Sigma} \preceq \frac{1}{1-b} \boldsymbol{I}
$$

We later transform the more interesting case with an unknown mean and an unknown diagonal covariance matrix to the isotropic case.

Theorem 2. Let $\mathcal{N}\left(\boldsymbol{\mu}^{*}, \boldsymbol{\Sigma}^{*}\right)$ be a d-dimensional Gaussian distribution that is in $\left(O\left(\log \left(1 / \alpha^{*}\right), 1 / 16\right)\right.$ isotropic position and consider a set $S$ such that $\mathcal{N}\left(\mu^{*}, \Sigma^{*} ; S\right) \geq \alpha$. There exists an algorithm such that for all $\epsilon>0$, the algorithm uses $n>d^{\text {poly }(1 / \alpha) \frac{\Gamma^{2}(S)}{\varepsilon^{8}}}$ samples and produces, in poly $(n)$ time, estimates that, with probability at least $99 \%$, satisfy $d_{T V}\left(\mathcal{N}\left(\mu^{*}, \Sigma^{*}\right), \mathcal{N}(\hat{\mu}, \hat{\Sigma})\right) \leq \varepsilon$.

We can apply this theorem to estimate the parameters of any Gaussian distribution with an unknown mean and an unknown diagonal covariance matrix by bringing the Gaussian to an $\left(O\left(\log \left(1 / \alpha^{*}\right), 1 / 16\right)\right.$-isotropic position. Lemma 18 shows that with high probability, we can obtain initial estimates $\widetilde{\boldsymbol{\mu}}_{S}$ and $\widetilde{\boldsymbol{\Sigma}}_{S}$ so that $\left\|\boldsymbol{\Sigma}^{-1 / 2}\left(\widetilde{\boldsymbol{\mu}}_{S}-\boldsymbol{\mu}^{*}\right)\right\|_{2}^{2} \leq O\left(\log \frac{1}{\alpha}\right)$ and

$$
\widetilde{\Sigma}_{S} \succeq \Omega\left(\alpha^{2}\right) \Sigma^{*}, \quad \text { and } \quad\left\|\Sigma^{*-1 / 2} \widetilde{\Sigma}_{S} \Sigma^{*-1 / 2}-I\right\|_{F}^{2} \leq O\left(\log \frac{1}{\alpha}\right) .
$$

Given these estimates, we can transform the space so that $\widetilde{\boldsymbol{\mu}}_{S}=0$, and $\widetilde{\boldsymbol{\Sigma}}_{S}=\boldsymbol{I}$. We note that after this transformation, the mean will be at the right distance from 0 , while the eigenvalues $\lambda_{i}$ of $\Sigma^{*}$ will all be within the desired range $\frac{15}{16} \leq \lambda_{i} \leq \frac{16}{15}$ apart from at most $O(\log (1 / \alpha))$. This is because the condition $\left\|\Sigma^{*-1 / 2} \widetilde{\boldsymbol{\Sigma}}_{S} \boldsymbol{\Sigma}^{*-1 / 2}-\boldsymbol{I}\right\|_{F}^{2} \leq O\left(\log \frac{1}{\alpha}\right)$ implies that $\sum_{i}\left(1-\frac{1}{\lambda_{i}}\right)^{2} \leq O(\log (1 / \alpha))$. With this observation, since we know of the eigenvectors of $\Sigma^{*}$, we would be able to search over all possible corrections to the eigenvalues to bring the Gaussian in $\left(O(\log (1 / \alpha)), \frac{1}{16}\right)$-isotropic position as required by Theorem 2. We only need to correct $O(\log (1 / \alpha))$ of them.

We can form a space of candidate hypotheses for the underlying distribution, for each choice of $O(\log (1 / \alpha))$ out of the $d$ vectors along with the all possible scalings. These hypotheses are at most $d^{O(\log (1 / \alpha))}$ times $(\log (1 / \alpha))^{O(\log (1 / \alpha))}$ for all possible scalings. Thus, there are at most $d^{O(\log (1 / \alpha))}$ hypotheses. Running the algorithm for each one of them, we would learn at least one distribution and one set that is accurate according to the guarantees of Theorems 2. Running the generic hypothesis testing algorithm of Lemma 20, we can identify one that is closest in total variation distance to the true distribution $\mathcal{N}_{S}^{*}$. The sample complexity and runtime would thus only increase by at most $d^{O(\log (1 / \alpha))}$. As we showed in Lemma 3, knowing the truncated Gaussian in total variation distance suffices to learn in accuracy $\varepsilon$ the parameters of the untruncated distribution. We thus obtain as corollary, that we can estimate the parameters when the covariance is spherical or diagonal. The same results hold when one wants to recover the underlying set in these cases.

\subsection{Learning a Weighted Characteristic Function}

Our goal in this section is to recover using conditional samples from $\mathcal{N}_{S}^{*}$ a weighted characteristic function of the set $S$. In particular, we will show that it is possible to learn a good approximation 
to the function

$$
\psi(x)=\frac{\mathbf{1}_{S}(\boldsymbol{x})}{\alpha^{*}} \frac{\mathcal{N}\left(\boldsymbol{\mu}^{*}, \boldsymbol{\Sigma}^{*} ; \boldsymbol{x}\right)}{\mathcal{N}(\mathbf{0}, \mathbf{I} ; \boldsymbol{x})}=\frac{\mathbf{1}_{S}(\boldsymbol{x})}{\alpha^{*}} \frac{\mathcal{N}^{*}(\boldsymbol{x})}{\mathcal{N}_{0}(\boldsymbol{x})} .
$$

We will later use the knowledge of this function to extract the unknown parameters and learn the set $S$.

\subsubsection{Hermite Concentration}

We start by showing that the function $\psi(x)$ admits strong Hermite concentration. This means that we can well-approximate $\psi(x)$ if we ignore the higher order terms in the Hermite expansion of $\psi(x)$.

Theorem 3. (Low Degree Approximation) Let $S_{k} \psi$ denote the degree $k$ Hermite expansion of function $\psi$ defined in (4). We have that

$$
\underset{x \sim \mathcal{N}_{0}}{\mathbb{E}}\left[\left(S_{k} \psi(x)-\psi(x)\right)^{2}\right]=\sum_{|V| \geq k} \hat{\psi}(V)^{2} \leq \operatorname{poly}(1 / \alpha)\left(\frac{\sqrt{\Gamma(S)}}{k^{1 / 4}}+\frac{1}{k}\right) .
$$

where $\Gamma(S)$ is the Gaussian surface area of $S$, and $a<\alpha^{*}$ is the absolute constant of (3).

We note that the Hermite expansion of $\psi$ is well-defined as $\psi(x) \in L_{2}\left(\mathbb{R}^{d}, \mathcal{N}_{0}\right)$. This can be seen from the following lemma which will be useful in many calculations throughout the paper.

Lemma 4. Let $\mathcal{N}\left(\boldsymbol{\mu}_{1}, \boldsymbol{\Sigma}_{1}\right)$ and $\mathcal{N}\left(\boldsymbol{\mu}_{2}, \boldsymbol{\Sigma}_{2}\right)$ be two $\left(B, \frac{1-\delta}{2 k}\right)$-isotropic Gaussians for some parameters $B, \delta>0$ and $k \in \mathbb{N}$. It holds

$$
\exp \left(-\frac{13 k^{2}}{\delta} B\right) \leq \underset{x \sim \mathcal{N}_{0}}{\mathbb{E}}\left[\left(\frac{\mathcal{N}\left(\boldsymbol{\mu}_{1}, \boldsymbol{\Sigma}_{1} ; \boldsymbol{x}\right)}{\mathcal{N}\left(\boldsymbol{\mu}_{2}, \boldsymbol{\Sigma}_{2} ; \boldsymbol{x}\right)}\right)^{k}\right] \leq \exp \left(\frac{13 k^{2}}{\delta} B\right) .
$$

Lemma 4 applied for $\mathcal{N}_{0}$ and $\mathcal{N}^{*}$ for $k=2$ implies that $\psi(\boldsymbol{x}) \in L_{2}\left(\mathbb{R}^{d}, \mathcal{N}_{0}\right)$.

To get the desired bound for Theorem 3 we use the following lemma, which allows us to bound the Hermite concentration of a function $f$ through its noise stability.

Lemma 5. For any function $f: \mathbb{R}^{d} \mapsto \mathbb{R}$ and parameter $\rho \in(0,1)$, it holds

$$
\sum_{|V| \geq 1 / \rho} \hat{f}(V)^{2} \leq 2 \underset{x \sim \mathcal{N}(0, I)}{\mathbb{E}}\left[f(x)^{2}-f(x) T_{1-\rho} f(x)\right]
$$

Lemma 5 was originally shown in [KKMS05] for indicator functions of sets, but their proof extends to arbitrary real functions. We provide the proof in the appendix for completeness.

Using Lemma 5, we can obtain Theorem 3 by bounding the noise sensitivity of the function $\psi$. The following lemma directly gives the desired result.

Lemma 6. For any $\rho \in(0,1), \mathbb{E}_{x \sim \mathcal{N}_{0}}\left[\psi(x)^{2}-\psi(x) T_{1-\rho} \psi(x)\right] \leq \operatorname{poly}(1 / \alpha)\left(\sqrt{\Gamma(S)} \rho^{1 / 4}+\rho\right)$.

To prove Lemma 6, we will require the following lemma whose proof is provided in the appendix. 
Lemma 7. Let $r(\boldsymbol{x}) \in L_{2}\left(\mathbb{R}^{d}, \mathcal{N}(\mathbf{0}, \boldsymbol{I})\right)$ be differentiable at every $\boldsymbol{x} \in \mathbb{R}^{d}$. Then

$$
\frac{1}{2} \underset{(x, z) \sim D_{\rho}}{\mathbb{E}}\left[(r(\boldsymbol{x})-r(\boldsymbol{z}))^{2}\right] \leq \rho \underset{x \sim \mathcal{N}(\mathbf{0}, \boldsymbol{I})}{\mathbb{E}}\left[\|\nabla r(\boldsymbol{x})\|_{2}^{2}\right]
$$

We now move on to the proof of Lemma 6.

Proof of Lemma 6: For ease of notation we define the following distribution

$$
D_{\rho}=\mathcal{N}\left(\mathbf{0},\left(\begin{array}{cc}
\boldsymbol{I} & (1-\rho) \boldsymbol{I} \\
(1-\rho) \boldsymbol{I} & \boldsymbol{I}
\end{array}\right)\right) .
$$

We also denote by $r(x)=\mathcal{N}^{*}(x) / \mathcal{N}_{0}(\boldsymbol{x})$ We can now write

$$
\begin{aligned}
2 \underset{x \sim \mathcal{N}_{0}}{\mathbb{E}}\left[\psi(\boldsymbol{x})^{2}-\psi(\boldsymbol{x}) T_{1-\rho} \psi(\boldsymbol{x})\right] & =\underset{(\boldsymbol{x}, \boldsymbol{z}) \sim D_{\rho}}{\mathbb{E}}\left[\psi(\boldsymbol{x})^{2}-\psi(\boldsymbol{x}) \psi(\boldsymbol{z})\right] \\
& =\frac{1}{\alpha^{* 2}} \underset{(\boldsymbol{x}, \boldsymbol{z}) \sim D_{\rho}}{\mathbb{E}}\left[\mathbf{1}_{S}(\boldsymbol{x}) r^{2}(\boldsymbol{x})-\mathbf{1}_{S}(\boldsymbol{x}) \mathbf{1}_{S}(\boldsymbol{z}) r^{2}(\boldsymbol{x})\right]+ \\
& \underset{(\boldsymbol{x}, \boldsymbol{z}) \sim D_{\rho}}{\mathbb{E}}\left[\mathbf{1}_{S}(\boldsymbol{x}) \mathbf{1}_{S}(\boldsymbol{z}) r^{2}(\boldsymbol{x})-\mathbf{1}_{S}(\boldsymbol{x}) \mathbf{1}_{S}(\boldsymbol{z}) r(\boldsymbol{x}) r(\boldsymbol{z})\right]
\end{aligned}
$$

We bound each of the two terms separately. For the first term, using Schwarz's inequality we get

$$
\begin{aligned}
\underset{(x, z) \sim D_{\rho}}{\mathbb{E}}\left[\mathbf{1}_{S}(\boldsymbol{x}) r^{2}(\boldsymbol{x})-\mathbf{1}_{S}(\boldsymbol{x}) \mathbf{1}_{S}(\boldsymbol{z}) r^{2}(\boldsymbol{x})\right] & \leq\left(\underset{(x, z) \sim D_{\rho}}{\mathbb{E}}\left[\mathbf{1}_{S}(\boldsymbol{x}) \mathbf{1}_{\bar{S}}(\boldsymbol{z})\right]\right)^{1 / 2}\left(\underset{(x, \boldsymbol{z}) \sim D_{\rho}}{\mathbb{E}}\left[r^{4}(\boldsymbol{x})\right]\right)^{1 / 2} \\
& \leq(\mathbf{N S}[S])^{1 / 2} \operatorname{poly}(1 / \alpha) \leq \sqrt{\Gamma(S)} \rho^{1 / 4} \operatorname{poly}(1 / \alpha)
\end{aligned}
$$

where the bound on the expectation of $r^{4}(x)$ follows from Lemma 4 and the last inequality follows from Lemma 19.

For the second term, we have that

$$
\begin{aligned}
\underset{(x, z) \sim D_{\rho}}{\mathbb{E}}\left[\mathbf{1}_{S}(\boldsymbol{x}) \mathbf{1}_{S}(\boldsymbol{z})\left(r^{2}(\boldsymbol{x})-r(\boldsymbol{x}) r(\boldsymbol{z})\right)\right] & =\underset{(\boldsymbol{x}, \boldsymbol{z}) \sim D_{\rho}}{\mathbb{E}}\left[\mathbf{1}_{S}(\boldsymbol{x}) \mathbf{1}_{S}(\boldsymbol{z})\left(\frac{r^{2}(\boldsymbol{x})}{2}+\frac{r^{2}(\boldsymbol{z})}{2}-r(\boldsymbol{x}) r(\boldsymbol{z})\right)\right] \\
& =\underset{(\boldsymbol{x}, \boldsymbol{z}) \sim D_{\rho}}{\mathbb{E}}\left[\mathbf{1}_{S}(\boldsymbol{x}) \mathbf{1}_{S}(\boldsymbol{z}) \frac{1}{2}(r(\boldsymbol{x})-r(\boldsymbol{z}))^{2}\right] \\
& \leq \frac{1}{2} \underset{(\boldsymbol{x}, \boldsymbol{z}) \sim D_{\rho}}{\mathbb{E}}\left[(r(\boldsymbol{x})-r(\boldsymbol{z}))^{2}\right] \leq \rho \underset{\boldsymbol{x} \sim N_{0}}{\mathbb{E}}\left[\|\nabla r(\boldsymbol{x})\|_{2}^{2}\right],
\end{aligned}
$$

where the last inequality follows from Lemma 7. It thus suffices to bound the expectation of the gradient of $r$. We have

$$
\begin{aligned}
\underset{x \sim N_{0}}{\mathbb{E}}\left[\|\nabla r(\boldsymbol{x})\|_{2}^{2}\right] & =\underset{x \sim N_{0}}{\mathbb{E}}\left[\left\|-\boldsymbol{\Sigma}^{*-1}\left(\boldsymbol{x}-\boldsymbol{\mu}^{*}\right)+\boldsymbol{x}\right\|_{2}^{2} r^{2}(\boldsymbol{x})\right] \\
& \leq 2 \underset{\boldsymbol{x} \sim N_{0}}{\mathbb{E}}\left[\left\|\left(\boldsymbol{I}-\boldsymbol{\Sigma}^{*}\right)^{-1} \boldsymbol{x}\right\|_{2}^{2} r^{2}(\boldsymbol{x})\right]+2\left\|\Sigma^{*-1} \boldsymbol{\mu}^{*}\right\|_{2}^{2} \underset{\boldsymbol{x} \sim N_{0}}{\mathbb{E}}\left[r^{2}(\boldsymbol{x})\right] \\
& \leq 2 \sqrt{\underset{x \sim N_{0}}{\mathbb{E}}\left[\left\|\left(\boldsymbol{I}-\boldsymbol{\Sigma}^{*-1}\right) \boldsymbol{x}\right\|_{2}^{4}\right] \underset{x \sim N_{0}}{\mathbb{E}}\left[r^{4}(\boldsymbol{x})\right]}+2\left\|\Sigma^{*-1} \boldsymbol{\mu}^{*}\right\|_{2}^{2} \underset{x \sim N_{0}}{\mathbb{E}}\left[r^{2}(\boldsymbol{x})\right] \leq \operatorname{poly}(1 / \alpha)
\end{aligned}
$$

where the bound on the expectation of $r^{4}(\boldsymbol{x})$ and $r^{2}(\boldsymbol{x})$ follows from Lemma 4 and the expectation

$$
\underset{x \sim N_{0}}{\mathbb{E}}\left[\left\|\left(\boldsymbol{I}-\boldsymbol{\Sigma}^{*-1}\right) \boldsymbol{x}\right\|_{2}^{4}\right]=\underset{x \sim N_{0}}{\mathbb{E}}\left[\left(\sum_{i}\left(1-\lambda_{i}\right)^{2} x_{i}^{2}\right)^{2}\right] \leq 3\left(\sum_{i}\left(1-\lambda_{i}\right)^{2}\right)^{2} \leq 3 \log ^{2}(1 / \alpha) \leq \operatorname{poly}(1 / \alpha)
$$




\subsubsection{Learning the Hermite Expansion}

In this section we deal with the sample complexity of estimating the coefficients of the Hermite expansion. We have

$$
c_{V}=\underset{x \sim \mathcal{N}(\mu, \Sigma, S)}{\mathbb{E}}\left[H_{V}(\boldsymbol{x})\right]
$$

Using samples $x_{i}$ from $\mathcal{N}(\mu, \Sigma, S)$, we can estimate this expectation empirically with the unbiased estimate

$$
\widetilde{c}_{V}=\frac{\sum_{i=1}^{N} H_{V}\left(\boldsymbol{x}_{i}\right)}{N} .
$$

We now show an upper bound for the variance of the above estimate. The proof of this lemma can be found in Appendix C.

Lemma 8. Let $\mathcal{N}\left(\boldsymbol{\mu}^{*}, \boldsymbol{\Sigma}^{*}, S\right)$ be the unknown truncated Gaussian. The variance of the following unbiased estimator of the Hermite coefficients $\widetilde{c}_{V}=\frac{\sum_{i=1}^{N} H_{V}\left(x_{i}\right)}{N}$, is upper bounded

$$
\underset{x \sim \mathcal{N}(\mu, \Sigma, S)}{\mathbb{E}}\left[\left(\widetilde{c}_{V}-c_{V}\right)^{2}\right] \leq \operatorname{poly}(1 / \alpha) \frac{5^{|V|}}{N} .
$$

Theorem 4. Let $S$ be an arbitrary (Borel) subset of $\mathbb{R}^{d}$. Let $\alpha$ be the constant of (3). Let $\mathcal{N}\left(\boldsymbol{\mu}^{*}, \boldsymbol{\Sigma}^{*}, S\right)$ be the corresponding truncated Gaussian in $(O \log (1 / \alpha), 1 / 16)$-isotropic position (see Definition 2), Then, for the estimate

$$
\psi_{k}(\boldsymbol{x})=\max \left(0, \sum_{V: 0 \leq|V| \leq k} \widetilde{\boldsymbol{c}}_{V} H_{V}(\boldsymbol{x})\right), \quad \widetilde{\boldsymbol{c}}_{V}=\frac{\sum_{i=1}^{N} H_{V}\left(\boldsymbol{x}_{i}\right)}{N}
$$

it holds for $k \ll d, \Gamma(S)>1$,

$$
\underset{x_{1}, \ldots, x_{N} \sim \mathcal{N}\left(\boldsymbol{\mu}^{*}, \boldsymbol{\Sigma}^{*}, S\right)}{\mathbb{E}}\left[\underset{\boldsymbol{x} \sim \mathcal{N}(\mathbf{0}, \boldsymbol{I})}{\mathbb{E}}\left[\left(\psi_{k}(\boldsymbol{x})-\psi(\boldsymbol{x})\right)^{2}\right]\right] \leq \operatorname{poly}(1 / \alpha)\left(\frac{\sqrt{\Gamma(S)}}{k^{1 / 4}}+\frac{(5 d)^{k}}{N}\right) .
$$

Alternatively, for $k=\operatorname{poly}(1 / \alpha) \Gamma(S)^{2} / \varepsilon^{4}$ we obtain that with $N=d^{\operatorname{poly}(1 / \alpha) \Gamma(S)^{2} / \varepsilon^{4}}$ samples, with probability at least $9 / 10$, it holds $\mathbb{E}_{\boldsymbol{x} \sim \mathcal{N}_{0}}\left[\left(\psi_{N, k}(\boldsymbol{x})-\psi(\boldsymbol{x})\right)^{2}\right] \leq \varepsilon$.

Proof. Instead of considering the positive part of the Hermite expansion, we will prove the claim for the empirical Hermite expansion of degree $k$ and $N$ samples

$$
p_{N, k}=\sum_{V: 0 \leq|V| \leq k} \widetilde{\mathcal{c}}_{V} H_{V}(\boldsymbol{x})
$$

As usual we denote by $S_{k} \psi(x)$ the true (exact) Hermite expansion of degree $k$ of $\psi(x)$. Using the inequality $(a-b)^{2} \leq 2(a-c)^{2}+2(c-b)^{2}$ we obtain

$$
\underset{x \sim \mathcal{N}_{0}}{\mathbb{E}}\left[\left(p_{N, k}(\boldsymbol{x})-f(\boldsymbol{x})\right)^{2}\right] \leq 2 \underset{\boldsymbol{x} \sim \mathcal{N}_{0}}{\mathbb{E}}\left[\left(p_{N, k}(\boldsymbol{x})-S_{k} \psi(\boldsymbol{x})\right)^{2}\right]+2 \underset{\boldsymbol{x} \sim \mathcal{N}_{0}}{\mathbb{E}}\left[\left(S_{k} \psi(\boldsymbol{x})-\psi(\boldsymbol{x})\right)^{2}\right]
$$

Since Hermite polynomials form an orthonormal system with respect to $\mathcal{N}_{0}$, we obtain

$$
\underset{x \sim \mathcal{N}_{0}}{\mathbb{E}}\left[\left(p_{N, k}(\boldsymbol{x})-S_{k} \psi(\boldsymbol{x})\right)^{2}\right]=\underset{x \sim \mathcal{N}_{0}}{\mathbb{E}}\left[\left(\sum_{V: 0 \leq|V| \leq k}\left(\widetilde{c}_{V}-c_{V}\right) H_{V}(\boldsymbol{x})\right)^{2}\right]=\sum_{V: 0 \leq|V| \leq k}\left(\widetilde{c}_{V}-c_{V}\right)^{2}
$$


Using Lemma 8 we obtain

$$
\underset{x_{1}, \ldots, x_{N} \sim \mathcal{N}^{*}}{\mathbb{E}}\left[\sum_{V: 0 \leq|V| \leq k}\left(\widetilde{c}_{V}-c_{V}\right)^{2}\right] \leq \frac{\operatorname{poly}(1 / \alpha)}{N} \sum_{V: 0 \leq|V| \leq k} 5^{|V|} \leq \frac{\operatorname{poly}(1 / \alpha)}{N}\left(\begin{array}{c}
d+k \\
k
\end{array}\right) 5^{k}
$$

where we used the fact that the number of all multi-indices $V$ of $d$ elements such that $0 \leq|V| \leq k$ is $\left(\begin{array}{c}d+k \\ k\end{array}\right)$. Moreover, from Theorem 3 we obtain that

$$
\underset{x \sim \mathcal{N}_{0}}{\mathbb{E}}\left[\left(S_{k} \psi(\boldsymbol{x})-\psi(\boldsymbol{x})\right)^{2}\right] \leq \operatorname{poly}(1 / \alpha)\left(\frac{\sqrt{\Gamma(S)}}{k^{1 / 4}}+\frac{1}{k}\right) .
$$

The theorem follows.

\subsection{Optimization of Gaussian Parameters}

In this section we show that we can formulate a convex objective function that can be optimized to yield the unknown parameters $\mu^{*}, \Sigma^{*}$ of the truncated Gaussian. Let $S$ be the unknown (Borel) subset of $\mathbb{R}^{d}$ such that $\mathcal{N}\left(\boldsymbol{\mu}^{*}, \boldsymbol{\Sigma}^{*} ; S\right)=\alpha^{*}$ and let $\mathcal{N}_{S}^{*}=\mathcal{N}\left(\boldsymbol{\mu}^{*}, \boldsymbol{\Sigma}^{*}, S\right)$ be the corresponding truncated Gaussian.

To find the parameters $\mu^{*}, \Sigma^{*}$, we define the function

$$
M_{f}(\boldsymbol{u}, \boldsymbol{B}) \triangleq \underset{\boldsymbol{x} \sim \mathcal{N}_{S}^{*}}{\mathbb{E}}\left[\mathrm{e}^{h(\boldsymbol{u}, \boldsymbol{B} ; \boldsymbol{x})} \mathcal{N}(\mathbf{0}, \boldsymbol{I} ; \boldsymbol{x}) f(\boldsymbol{x})\right]
$$

where $h(\boldsymbol{u}, \boldsymbol{B} ; \boldsymbol{x})=\frac{\boldsymbol{x}^{T} \boldsymbol{B} \boldsymbol{x}}{2}-\frac{\operatorname{tr}\left((\boldsymbol{B}-\boldsymbol{I})\left(\widetilde{\boldsymbol{\Sigma}}_{S}+\widetilde{\boldsymbol{\mu}}_{S} \widetilde{\boldsymbol{\mu}}_{S}^{T}\right)\right)}{2}-\boldsymbol{u}^{T}\left(\boldsymbol{x}-\widetilde{\boldsymbol{\mu}}_{S}\right)+\frac{d}{2} \log 2 \pi$.

We will show that the minimizer of $M_{f}(\boldsymbol{u}, \boldsymbol{B})$ for the polynomial function $f=\psi_{k}$, will satisfy $\left(\boldsymbol{B}^{-1} \boldsymbol{u}, \boldsymbol{B}^{-1}\right) \approx\left(\boldsymbol{\mu}^{*}, \boldsymbol{\Sigma}^{*}\right)$. Note that $M_{f}(\boldsymbol{u}, \boldsymbol{B})$ can be estimated through samples. Our goal will be to optimize it through stochastic gradient descent.

In order to make sure that SGD algorithm for $M_{\psi_{k}}$ converges fast in the parameter space we need to project after every iteration to some subset of the space as we will see in more details later in this Section. Assuming that the pair $\left(\boldsymbol{\mu}^{*}, \boldsymbol{\Sigma}^{*}\right)$ is in $\left(\sqrt{\log \left(1 / \alpha^{*}\right)}, 1 / 16\right)$-isotropic position we define the following set

$$
\mathcal{D}=\left\{(\boldsymbol{u}, \boldsymbol{B}) \mid\left(\boldsymbol{B}^{-1} \boldsymbol{u}, \boldsymbol{B}^{-1}\right) \text { is in }\left(c \cdot \log \left(1 / \alpha^{*}\right), 1 / 16\right) \text {-isotropic position }\right\}
$$

Where $c$ is the universal constant guaranteed to exist from Section 2.1 so that

$$
\max \left\{\left\|\mu^{*}-\tilde{\mu}\right\|_{\Sigma^{*}},\left\|\Sigma^{*}-\tilde{\Sigma}\right\|_{F}\right\} \leq c \cdot \log \left(1 / \alpha^{*}\right) .
$$

It is not hard to see that $\mathcal{D}$ is a convex set and that for any $(\boldsymbol{u}, \boldsymbol{B})$ the projection to $\mathcal{D}$ can be done efficiently. For more details we refer to Lemma 8 of [DGTZ18]. Since after every iteration of our algorithm we project to $\mathcal{D}$ we will assume for the rest of this Section that $(\boldsymbol{u}, \boldsymbol{B}) \in \mathcal{D}$.

An equivalent formulation of $M_{f}(\boldsymbol{u}, \boldsymbol{B})$ that will be useful for the analysis of the SGD algorithm is

$$
\begin{aligned}
M_{f}(\boldsymbol{u}, \boldsymbol{B}) & =e^{\left.-\frac{1}{2}\left(\operatorname{tr}\left((\boldsymbol{B}-\boldsymbol{I})\left(\widetilde{\boldsymbol{\Sigma}}_{S}+\widetilde{\boldsymbol{\mu}}_{S} \widetilde{\boldsymbol{\mu}}_{S}^{T}\right)\right)\right)+\boldsymbol{u}^{T} \boldsymbol{B}^{-1} \boldsymbol{u}-\boldsymbol{u}^{T} \widetilde{\boldsymbol{\mu}}_{S}\right)} \sqrt{|\boldsymbol{B}|} \underset{\boldsymbol{x} \sim \mathcal{N}_{S}^{*}}{\mathbb{E}}\left[\frac{\mathcal{N}(\mathbf{0}, \boldsymbol{I} ; \boldsymbol{x})}{\mathcal{N}\left(\boldsymbol{B}^{-1} \boldsymbol{u}, \boldsymbol{B}^{-1} ; \boldsymbol{x}\right)} f(\boldsymbol{x})\right] \\
& \triangleq C_{\boldsymbol{u}, \boldsymbol{B}} \underset{\boldsymbol{x} \sim \mathcal{N}_{S}^{*}}{\mathbb{E}}\left[\frac{\mathcal{N}_{0}(\boldsymbol{x})}{\mathcal{N}_{\boldsymbol{u}, \boldsymbol{B}}(\boldsymbol{x})} f(\boldsymbol{x})\right]
\end{aligned}
$$


Lemma 9. For $(\boldsymbol{u}, \boldsymbol{B}) \in \mathcal{D}$, we have that $\operatorname{poly}(\alpha) \leq C_{\boldsymbol{u}, \boldsymbol{B}} \leq \operatorname{poly}(1 / \alpha)$.

Proof. We have that

$$
\begin{aligned}
\left|2 \log C_{\boldsymbol{u}, \boldsymbol{B}}\right| & \left.=\mid \operatorname{tr}\left((\boldsymbol{B}-\boldsymbol{I})\left(\widetilde{\boldsymbol{\Sigma}}_{S}+\widetilde{\boldsymbol{\mu}}_{S} \widetilde{\boldsymbol{\mu}}_{S}^{T}\right)\right)\right)+\boldsymbol{u}^{T} \boldsymbol{B}^{-1} \boldsymbol{u}-\boldsymbol{u}^{T} \widetilde{\boldsymbol{\mu}}_{S}-\log |\boldsymbol{B}| \mid \\
& =\left|\operatorname{tr}(\boldsymbol{B}-\boldsymbol{I})+\operatorname{tr}\left((\boldsymbol{B}-\boldsymbol{I})\left(\widetilde{\boldsymbol{\Sigma}}_{S}-\boldsymbol{I}\right)\right)+\boldsymbol{u}^{T} \boldsymbol{B}^{-1} \boldsymbol{u}-\log \right| \boldsymbol{B}|| \\
& \leq|\operatorname{tr}(\boldsymbol{B}-\boldsymbol{I})-\log | \boldsymbol{B}||+\left|\operatorname{tr}\left((\boldsymbol{B}-\boldsymbol{I})\left(\widetilde{\boldsymbol{\Sigma}}_{S}-\boldsymbol{I}\right)\right)\right|+\left|\boldsymbol{u}^{T} \boldsymbol{B}^{-1} \boldsymbol{u}\right|
\end{aligned}
$$

We now bound each of the terms separately. Let $\lambda_{1}, \ldots, \lambda_{d}$ be the eigenvalues of $\boldsymbol{B}$.

1. For the first term, we have that

$$
|\operatorname{tr}(\boldsymbol{B}-\boldsymbol{I})-\log | \boldsymbol{B}||=\left|\sum_{i=1}^{d}\left(\lambda_{i}-1-\log \lambda_{i}\right)\right| \leq \sum_{i=1}^{d} \frac{\left(\lambda_{i}-1\right)^{2}}{\lambda_{i}} \leq \frac{\|\boldsymbol{B}-\boldsymbol{I}\|_{F}^{2}}{\lambda_{\min }}
$$

where we used the fact that $0 \leq x-1-\log x \leq \frac{(x-1)^{2}}{x}$ for all $x>0$.

2. For the second term, we have that $\left|\operatorname{tr}\left((\boldsymbol{B}-\boldsymbol{I})\left(\widetilde{\boldsymbol{\Sigma}}_{S}-\boldsymbol{I}\right)\right)\right| \leq\|\boldsymbol{B}-\boldsymbol{I}\|_{F}\left\|\widetilde{\boldsymbol{\Sigma}}_{S}-\boldsymbol{I}\right\|_{F}$

3. For the third term, we have that $\left|\boldsymbol{u}^{T} \boldsymbol{B}^{-1} \boldsymbol{u}\right|=\boldsymbol{u}^{T} \boldsymbol{B}^{-1} \boldsymbol{B} \boldsymbol{B}^{-1} \boldsymbol{u} \leq \lambda_{\text {max }}\left\|\boldsymbol{B}^{-1} \boldsymbol{u}\right\|_{2}^{2}$

Now from the assumption $(\boldsymbol{u}, \boldsymbol{B}) \in \mathcal{D}$ we have that $\|\boldsymbol{B}-\boldsymbol{I}\|_{F} \leq O\left(\sqrt{\log \left(1 / \alpha^{*}\right)}\right),\left\|\boldsymbol{B}^{-1} \boldsymbol{u}\right\|_{2} \leq$ $O\left(\sqrt{\log \left(1 / \alpha^{*}\right)}\right), \lambda_{\min } \geq 15 / 16$ and $\lambda_{\max } \leq 17 / 16$. Also from Lemma 18 we get that $\left\|\tilde{\boldsymbol{\Sigma}}_{S}-\boldsymbol{I}\right\|_{F} \leq$ $O\left(\sqrt{\log \left(1 / \alpha^{*}\right)}\right)$ and hence $\left|2 \log C_{\boldsymbol{u}, \boldsymbol{B}}\right| \leq O\left(\log \left(1 / \alpha^{*}\right)\right)$. This means that $C_{\boldsymbol{u}, \boldsymbol{B}}=\operatorname{poly}(1 / \alpha)$ and the lemma follows.

\subsubsection{The Objective Function and its Approximation}

To show that the minimizer of the function $M_{\psi_{k}}$ is a good estimator for the unknown parameters $\boldsymbol{\mu}^{*}, \boldsymbol{\Sigma}^{*}$, we consider the function $M_{f}^{\prime}$, defined as $M_{f}(\boldsymbol{u}, \boldsymbol{B})=\mathbb{E}_{\boldsymbol{x} \sim \mathcal{N}_{S}^{*}}\left[\mathrm{e}^{h^{\prime}(\boldsymbol{u}, \boldsymbol{B} ; \boldsymbol{x})} \mathcal{N}(\mathbf{0}, \boldsymbol{I} ; \boldsymbol{x}) f(\boldsymbol{x})\right]$ for $h^{\prime}(\boldsymbol{u}, \boldsymbol{B} ; \boldsymbol{x})=\frac{\boldsymbol{x}^{T} \boldsymbol{B} \boldsymbol{x}}{2}-\frac{\operatorname{tr}\left((\boldsymbol{B}-\boldsymbol{I})\left(\boldsymbol{\Sigma}_{S}+\boldsymbol{\mu}_{S} \boldsymbol{\mu}_{S}^{T}\right)\right)}{2}-\boldsymbol{u}^{T}\left(\boldsymbol{x}-\boldsymbol{\mu}_{S}\right)+\frac{d}{2} \log 2 \pi$. This function corresponds to an ideal situation where we know the parameters $\mu_{S}, \Sigma_{S}$ exactly. Similarly to (7), we can write $M_{f}^{\prime}$ as $C_{u, B}^{\prime} \mathbb{E}_{x \sim \mathcal{N}_{S}^{*}}\left[\frac{\mathcal{N}_{0}(x)}{\mathcal{N}_{u, B}(x)} f(x)\right]$. We argue that both $M_{f}$ and $M_{f}^{\prime}$ are convex.

Claim 1. For any function $f: \mathbb{R}^{d} \mapsto \mathbb{R}_{\geq 0}, M_{f}(\boldsymbol{u}, \boldsymbol{B})$ and $M_{f}^{\prime}(\boldsymbol{u}, \boldsymbol{B})$ are convex functions of the parameters $(\boldsymbol{u}, \boldsymbol{B})$.

Proof. We show the statement for $M_{f}$. The proof for $M_{f}^{\prime}$ is identical. The proof follows by computing the Hessian of $M_{f}$ and arguing that it is positive semidefinite.

The gradient with respect to $(\boldsymbol{u}, \boldsymbol{B})$ is

$$
\begin{aligned}
\nabla M_{f}(\boldsymbol{u}, \boldsymbol{B}) & =\underset{\boldsymbol{x} \sim \mathcal{N}\left(\boldsymbol{\mu}^{*}, \boldsymbol{\Sigma}^{*}, S\right)}{\mathbb{E}}\left[\nabla h(\boldsymbol{u}, \boldsymbol{B} ; \boldsymbol{x}) \mathrm{e}^{h(\boldsymbol{u}, \boldsymbol{B} ; \boldsymbol{x})} \mathcal{N}(\mathbf{0}, \boldsymbol{I} ; \boldsymbol{x}) f(\boldsymbol{x})\right] \\
& =\underset{\boldsymbol{x} \sim \mathcal{N}\left(\boldsymbol{\mu}^{*}, \boldsymbol{\Sigma}^{*}, S\right)}{\mathbb{E}}\left[\left(\begin{array}{c}
\frac{1}{2}\left(\boldsymbol{x} \boldsymbol{x}^{T}-\tilde{\boldsymbol{\Sigma}}_{S}-\tilde{\boldsymbol{\mu}}_{S} \tilde{\boldsymbol{\mu}}_{S}^{T}\right)^{b} \\
\tilde{\boldsymbol{\mu}}_{S}-\boldsymbol{x}
\end{array}\right) \mathrm{e}^{h(\boldsymbol{u}, \boldsymbol{B} ; \boldsymbol{x})} \mathcal{N}(\mathbf{0}, \boldsymbol{I} ; \boldsymbol{x}) f(\boldsymbol{x})\right]
\end{aligned}
$$


Moreover, the Hessian is

$\mathcal{H}_{M_{f}}(\boldsymbol{u}, \boldsymbol{B})=\underset{x \sim \mathcal{N}\left(\boldsymbol{\mu}^{*}, \boldsymbol{\Sigma}^{*}, S\right)}{\mathbb{E}}\left[\left(\begin{array}{c}\frac{1}{2}\left(x \boldsymbol{x}^{T}-\tilde{\boldsymbol{\Sigma}}_{S}-\tilde{\boldsymbol{\mu}}_{S} \tilde{\boldsymbol{\mu}}_{S}^{T}\right)^{b} \\ \tilde{\boldsymbol{\mu}}_{S}-\boldsymbol{x}\end{array}\right)\left(\begin{array}{c}\frac{1}{2}\left(\boldsymbol{x} \boldsymbol{x}^{T}-\tilde{\boldsymbol{\Sigma}}_{S}-\tilde{\boldsymbol{\mu}}_{S} \tilde{\boldsymbol{\mu}}_{S}^{T}\right)^{b} \\ \tilde{\boldsymbol{\mu}}_{S}-\boldsymbol{x}\end{array}\right)^{T} \mathrm{e}^{h(\boldsymbol{u}, \boldsymbol{B} ; \boldsymbol{x})} \mathcal{N}(\mathbf{0}, \boldsymbol{I} ; \boldsymbol{x}) f(\boldsymbol{x})\right]$

which is clearly positive semidefinite since for any $z \in \mathbb{R}^{d \times d+d}$ we have

$$
\boldsymbol{z}^{T} \mathcal{H}_{M_{f}}(\boldsymbol{u}, \boldsymbol{B}) \boldsymbol{z}=\underset{\boldsymbol{x} \sim \mathcal{N}\left(\boldsymbol{\mu}^{*}, \boldsymbol{\Sigma}^{*}, S\right)}{\mathbb{E}}\left[\left(\boldsymbol{z}^{T}\left(\begin{array}{c}
\frac{1}{2}\left(\boldsymbol{x} \boldsymbol{x}^{T}-\tilde{\boldsymbol{\Sigma}}_{S}-\tilde{\boldsymbol{\mu}}_{S} \tilde{\boldsymbol{\mu}}_{S}^{T}\right)^{b} \\
\tilde{\boldsymbol{\mu}}_{S}-\boldsymbol{x}
\end{array}\right)\right)^{2} \mathrm{e}^{h(\boldsymbol{u}, \boldsymbol{B} ; \boldsymbol{x})} \mathcal{N}(\mathbf{0}, \boldsymbol{I} ; \boldsymbol{x}) f(\boldsymbol{x})\right] \geq 0 .
$$

We now argue that the minimizer of the convex function $M_{\psi}^{\prime}$ for the weighted characteristic function $\psi(\boldsymbol{x})=\frac{\mathbf{1}_{S}(\boldsymbol{x})}{\alpha^{*}} \frac{\mathcal{N}\left(\boldsymbol{\mu}^{*}, \boldsymbol{\Sigma}^{*} ; \boldsymbol{x}\right)}{\mathcal{N}(\mathbf{0}, \mathbf{I} ; \boldsymbol{x})}$ is $(\boldsymbol{u}, \boldsymbol{B})=\left(\boldsymbol{\Sigma}^{*-1}, \boldsymbol{\Sigma}^{*-1} \boldsymbol{\mu}^{*}\right)$.

Claim 2. The minimizer of $M_{\psi}^{\prime}(\boldsymbol{u}, \boldsymbol{B})$ is $(\boldsymbol{u}, \boldsymbol{B})=\left(\boldsymbol{\Sigma}^{*-1}, \boldsymbol{\Sigma}^{*-1} \boldsymbol{\mu}^{*}\right)$.

Proof. The gradient of $M_{\psi}^{\prime}$ with respect to $(\boldsymbol{u}, \boldsymbol{B})$ is

$$
\begin{aligned}
\nabla M_{\psi}^{\prime}(\boldsymbol{u}, \boldsymbol{B}) & =\underset{\boldsymbol{x} \sim \mathcal{N}_{S}^{*}}{\mathbb{E}}\left[\left(\begin{array}{c}
\frac{1}{2}\left(\boldsymbol{x} \boldsymbol{x}^{T}-\boldsymbol{\Sigma}_{S}-\boldsymbol{\mu}_{S} \boldsymbol{\mu}_{S}^{T}\right)^{b} \\
\boldsymbol{\mu}_{S}-\boldsymbol{x}
\end{array}\right) \mathrm{e}^{h(u, \boldsymbol{B} ; \boldsymbol{x})} \mathcal{N}(\mathbf{0}, \mathbf{I} ; \boldsymbol{x}) \frac{\mathbf{1}_{S}(\boldsymbol{x})}{\alpha^{*}} \frac{\mathcal{N}\left(\boldsymbol{\mu}^{*}, \boldsymbol{\Sigma}^{*} ; \boldsymbol{x}\right)}{\mathcal{N}(\mathbf{0}, \mathbf{I} ; \boldsymbol{x})}\right] \\
& =\underset{x \sim \mathcal{N}_{S}^{*}}{\mathbb{E}}\left[\left(\begin{array}{c}
\frac{1}{2}\left(\boldsymbol{x} \boldsymbol{x}^{T}-\boldsymbol{\Sigma}_{S}-\boldsymbol{\mu}_{S} \boldsymbol{\mu}_{S}^{T}\right)^{b} \\
\boldsymbol{\mu}_{S}-x
\end{array}\right) \mathrm{e}^{h(\boldsymbol{u}, \boldsymbol{B} ; \boldsymbol{x})} \frac{\mathcal{N}\left(\boldsymbol{\mu}^{*}, \boldsymbol{\Sigma}^{*} ; \boldsymbol{x}\right)}{\alpha^{*}}\right]
\end{aligned}
$$

For $(\boldsymbol{u}, \boldsymbol{B})=\left(\boldsymbol{\Sigma}^{*-1} \boldsymbol{\mu}^{*}, \boldsymbol{\Sigma}^{*-1}\right)$, this is equal to

$$
\begin{aligned}
\nabla M_{\psi}^{\prime}\left(\boldsymbol{\Sigma}^{*-1} \boldsymbol{\mu}^{*}, \boldsymbol{\Sigma}^{*-1}\right) & =C_{\boldsymbol{u}, \boldsymbol{B}} \cdot \underset{x \sim \mathcal{N}_{S}^{*}}{\mathbb{E}}\left[\left(\begin{array}{c}
\frac{1}{2}\left(x x^{T}-\boldsymbol{\Sigma}_{S}-\boldsymbol{\mu}_{S} \boldsymbol{\mu}_{S}^{T}\right)^{b} \\
\boldsymbol{\mu}_{S}-x
\end{array}\right) \frac{1}{\mathcal{N}\left(\boldsymbol{\mu}^{*}, \boldsymbol{\Sigma}^{*} ; \boldsymbol{x}\right)} \frac{\mathcal{N}\left(\boldsymbol{\mu}^{*}, \boldsymbol{\Sigma}^{*} ; \boldsymbol{x}\right)}{\alpha^{*}}\right] \\
& =\frac{C_{u, B}}{\alpha^{*}} \cdot \underset{x \sim \mathcal{N}_{S}^{*}}{\mathbb{E}}\left[\left(\begin{array}{c}
\frac{1}{2}\left(x x^{T}-\boldsymbol{\Sigma}_{S}-\boldsymbol{\mu}_{S} \boldsymbol{\mu}_{S}^{T}\right)^{b} \\
\boldsymbol{\mu}_{S}-x
\end{array}\right)\right]
\end{aligned}
$$

where $C_{u, B}$ that does not depend on $x$. This is equal to 0 by definition of $\mu_{S}$ and $\Sigma_{S}$.

We want to show that the minimizer of $M_{\psi_{k}}$ is close to that of $M_{\psi}^{\prime}$. To do this, we bound the difference of the two functions pointwise. The proof of the following lemma is technical and can be found in Appendix D.

Lemma 10 (Pointwise Approximation of the Objective Function). Assume that we use Lemma 17 to estimate $\tilde{\mu}_{S}, \tilde{\boldsymbol{\Sigma}}_{S}$ with $\varepsilon=\frac{1}{\text { poly }\left(1 / \alpha^{*}\right)} \varepsilon^{\prime}$ and Theorem 4 with $\varepsilon=\frac{1}{p\left(1 / \alpha^{*}\right)} \varepsilon^{\prime 2}$ then

$$
\left|M_{\psi_{k}}(\boldsymbol{u}, \boldsymbol{B})-M_{\psi}^{\prime}(\boldsymbol{u}, \boldsymbol{B})\right| \leq \boldsymbol{\varepsilon}^{\prime} .
$$

Now that we have established that $M_{\psi_{k}}$ is a good approximation of $M_{\psi}^{\prime}$ we will prove that we can optimize $M_{\psi_{k}}$ and get a solution that is very close to the optimal solution of $M_{\psi}^{\prime}$. 


\subsubsection{Optimization of the Approximate Objective Function}

Our goal in this section is to prove that using sample access to $\mathcal{N}\left(\boldsymbol{\mu}^{*}, \boldsymbol{\Sigma}^{*}, S\right)$ we can find the minimum of the function $M_{\psi_{k}}$ defined in the previous section. First of all recall that $M_{\psi_{k}}$ can be written as an expectation over $\mathcal{N}\left(\mu^{*}, \Sigma^{*}, S\right)$ in the following way

$$
M_{\psi_{k}}(\boldsymbol{u}, \boldsymbol{B}) \triangleq \underset{\boldsymbol{x} \sim \mathcal{N}_{S}^{*}}{\mathbb{E}}\left[\mathrm{e}^{h(\boldsymbol{u}, \boldsymbol{B} ; \boldsymbol{x})} \mathcal{N}(\mathbf{0}, \boldsymbol{I} ; \boldsymbol{x}) \psi_{k}(\boldsymbol{x})\right] .
$$

In Section 4.1 we prove that we can learn the function $\psi_{k}$ and hence $M_{\psi_{k}}$ can be written as

$$
M_{\psi_{k}}(\boldsymbol{u}, \boldsymbol{B})=\underset{\boldsymbol{x} \sim \mathcal{N}_{\mathcal{S}}^{*}}{\mathbb{E}}\left[m_{\psi_{k}}(\boldsymbol{u}, \boldsymbol{B} ; \boldsymbol{x})\right]
$$

where $m_{\psi_{k}}(\boldsymbol{u}, \boldsymbol{B} ; \boldsymbol{x})=\mathrm{e}^{h(\boldsymbol{u}, \boldsymbol{B} ; \boldsymbol{x})} \mathcal{N}(\mathbf{0}, \boldsymbol{I} ; \boldsymbol{x}) \psi_{k}(\boldsymbol{x})$, and for any $\boldsymbol{u}, \boldsymbol{B}$ and $\boldsymbol{x}$ we can compute $m_{\psi_{k}}(\boldsymbol{u}, \boldsymbol{B} ; \boldsymbol{x})$. Since $M_{\psi_{k}}$ is convex we are going to use stochastic gradient descent to find its minimum. To prove the convergence of SGD and bound the number of steps that SGD needs to converge we will use the the formulation developed in Chapter 14 of [SSBD14]. To be able to use their results we have to define for any $(\boldsymbol{u}, \boldsymbol{B})$ a random vector $\boldsymbol{v}(\boldsymbol{u}, \boldsymbol{B})$ and prove the following

Unbiased Gradient Estimation

$$
\mathbb{E}[\boldsymbol{v}(\boldsymbol{u}, \boldsymbol{B})]=\nabla M_{\psi_{k}}
$$

Bounded Step Variance

$$
\mathbb{E}\left[\|\boldsymbol{v}(\boldsymbol{u}, \boldsymbol{B})\|_{2}^{2}\right] \leq \rho,
$$

Strong Convexity for any $z \in \mathcal{D}$ it holds

$$
\boldsymbol{z}^{T} \mathcal{H}_{M_{f}}(\boldsymbol{u}, \boldsymbol{B}) \boldsymbol{z} \geq \lambda
$$

We start with the definition of the random vector $\boldsymbol{v}$. Given a sample $x$ from $\mathcal{N}\left(\boldsymbol{\mu}^{*}, \Sigma^{*}, S\right)$, for any $(\boldsymbol{u}, \boldsymbol{B})$ we define

$$
\begin{aligned}
\boldsymbol{v}(\boldsymbol{u}, \boldsymbol{B}) & =\nabla_{\boldsymbol{u}, \boldsymbol{B}} m_{\psi_{k}}(\boldsymbol{u}, \boldsymbol{B} ; \boldsymbol{x}) \\
& =\left(\begin{array}{c}
\frac{1}{2}\left(\boldsymbol{x} \boldsymbol{x}^{T}-\tilde{\boldsymbol{\Sigma}}_{S}-\tilde{\boldsymbol{\mu}}_{S} \tilde{\boldsymbol{\mu}}_{S}^{T}\right)^{b} \\
\tilde{\boldsymbol{\mu}}_{S}-\boldsymbol{x}
\end{array}\right) \mathrm{e}^{h(\boldsymbol{u}, \boldsymbol{B} ; \boldsymbol{x})} \mathcal{N}(\mathbf{0}, \boldsymbol{I} ; \boldsymbol{x}) \psi_{k}(\boldsymbol{x})
\end{aligned}
$$

observe that the randomness of $v$ only comes from the random sample $x \sim \mathcal{N}\left(\boldsymbol{\mu}^{*}, \boldsymbol{\Sigma}^{*}, S\right)$. The fact that $\boldsymbol{v}(\boldsymbol{u}, \boldsymbol{B})$ is an unbiased estimator of $\nabla M_{f}(\boldsymbol{u}, \boldsymbol{B})$ follows directly from the fact calculation of $\nabla M_{f}(\boldsymbol{u}, \boldsymbol{B})$ in Section 4.2.1. For the other two properties that we need we have the following lemmas. The following lemma bounds the variance of the step of the SGD algorithm. It's rather technical proof can be found in Appendix D.

Lemma 11 (Bounded Step Variance). Let $\alpha$ be the constant of (3). For every $(\boldsymbol{u}, \boldsymbol{B}) \in \mathcal{D}$ it holds

$$
\underset{\boldsymbol{x} \sim \mathcal{N}_{S}^{*}}{\mathbb{E}}\left[\|\boldsymbol{v}(\boldsymbol{u}, \boldsymbol{B})\|_{2}^{2}\right] \leq \operatorname{poly}(1 / \alpha) \cdot d^{2 k},
$$

We are now going to prove the strong convexity of the objective function $M_{\psi_{k}}$. For this we are going to use a known anti-concentration result (Theorem 9) for polynomial functions over the Gaussian measure. See Appendix A.

The following lemma shows that our objective is strongly convex as long as the guess $\boldsymbol{u}, \boldsymbol{B}$ remains in the set $\mathcal{D}$. Its proof can be found in Appendix D. 
Lemma 12 (Strong Convexity). Let $\alpha$ be the absolute constant of (3). For every $(\boldsymbol{u}, \boldsymbol{B}) \in \mathcal{D}$, any $z \in \mathbb{R}^{d}$ such that $\|z\|_{2}=1$ and the first $d^{2}$ coordinated of $z$ correspond to a symmetric matrix, then

$$
\boldsymbol{z}^{T} \mathcal{H}_{M_{f}}(\boldsymbol{u}, \boldsymbol{B}) \boldsymbol{z} \geq \operatorname{poly}(\alpha)
$$

\subsubsection{Recovering the Unconditional Mean and Covariance}

The framework that we use for proving the fast convergence of our SGD algorithm is summarized in the following theorem and the following lemma.

Theorem 5 (Theorem 14.11 of [SSBD14].). Let $f: \mathbb{R}^{d} \rightarrow \mathbb{R}$. Assume that $f$ is $\lambda$-strongly convex, that $\mathbb{E}\left[\boldsymbol{v}^{(i)} \mid \boldsymbol{w}^{(i-1)}\right] \in \partial f\left(\boldsymbol{w}^{(i-1)}\right)$ and that $\mathbb{E}\left[\left\|\boldsymbol{v}^{(i)}\right\|_{2}^{2}\right] \leq \rho^{2}$. Let $\boldsymbol{w}^{*} \in \arg \min _{\boldsymbol{w} \in \mathcal{D}} f(\boldsymbol{w})$ be an optimal solution. Then,

$$
\mathbb{E}[f(\overline{\boldsymbol{w}})]-f\left(\boldsymbol{w}^{*}\right) \leq \frac{\rho^{2}}{2 \lambda T}(1+\log T),
$$

where $\overline{\boldsymbol{w}}$ is the output projected stochastic gradient descent with steps $\boldsymbol{v}^{(i)}$ and projection set $\mathcal{D}$ after $T$ iterations.

Lemma 13 (Lemma 13.5 of [SSBD14].). If $f$ is $\lambda$-strongly convex and $w^{*}$ is a minimizer of $f$, then, for any $w$ it holds that

$$
f(\boldsymbol{w})-f\left(\boldsymbol{w}^{*}\right) \geq \frac{\lambda}{2}\left\|\boldsymbol{w}-\boldsymbol{w}^{*}\right\|_{2}^{2} .
$$

Now we have all the ingredients to present the proof of Theorem 2.

Proof of Theorem 2: The estimation procedure starts by computing the polynomial function $\psi_{k}$ using $d^{\text {poly }\left(1 / \alpha^{*}\right) \frac{\Gamma^{2}(S)}{\varepsilon^{\prime 8}}}$ samples from $\mathcal{N}\left(\boldsymbol{\mu}^{*}, \boldsymbol{\Sigma}^{*}, S\right)$ as explained in Theorem 4 to get error poly $\left(\alpha^{*}\right) \varepsilon^{\prime 2}$. Then we compute $\tilde{\boldsymbol{\mu}}_{S}$ and $\tilde{\boldsymbol{\Sigma}}_{S}$ as explained in Section 2.1 with $\varepsilon=\frac{q\left(\alpha^{*}\right)}{8 p\left(1 / \alpha^{*}\right)}\left(\varepsilon^{\prime}\right)^{2}$ where $p$ comes from Lemma 10 and $q$ comes from Lemma 12. Our estimators for $\hat{\mu}, \hat{\Sigma}$ are the outputs of Algorithm 1.

We analyze the accuracy of our estimation by proving that the minimum of $M_{\psi_{k}}$ is close in the parameter space to the minimum of $M_{\psi}^{\prime}$. Let $\boldsymbol{u}^{\prime}, \boldsymbol{B}^{\prime}$ be the minimum of the convex function $M_{\psi}^{\prime}$ and $\boldsymbol{u}_{k}, \boldsymbol{B}_{k}$ be the minimum of the convex function $M_{\psi_{k}}$. Using Lemma 10 we have the following relations

$$
\left|M_{\psi}^{\prime}\left(\boldsymbol{u}^{\prime}, \boldsymbol{B}^{\prime}\right)-M_{\psi_{k}}\left(\boldsymbol{u}^{\prime}, \boldsymbol{B}^{\prime}\right)\right| \leq \varepsilon^{\prime}, \quad\left|M_{\psi}^{\prime}\left(\boldsymbol{u}_{k}, \boldsymbol{B}_{k}\right)-M_{\psi_{k}}\left(\boldsymbol{u}_{k}, \boldsymbol{B}_{k}\right)\right| \leq \varepsilon^{\prime}
$$

and also

$$
M_{\psi}^{\prime}\left(\boldsymbol{u}^{\prime}, \boldsymbol{B}^{\prime}\right) \leq M_{\psi}^{\prime}\left(\boldsymbol{u}_{k}, \boldsymbol{B}_{k}\right), \quad M_{\psi_{k}}\left(\boldsymbol{u}_{k}, \boldsymbol{B}_{k}\right) \leq M_{\psi_{k}}\left(\boldsymbol{u}^{\prime}, \boldsymbol{B}^{\prime}\right) .
$$

These relations imply that

$$
\begin{aligned}
\left|M_{\psi_{k}}\left(\boldsymbol{u}^{\prime}, \boldsymbol{B}^{\prime}\right)-M_{\psi_{k}}\left(\boldsymbol{u}_{k}, \boldsymbol{B}_{k}\right)\right| & =M_{\psi_{k}}\left(\boldsymbol{u}^{\prime}, \boldsymbol{B}^{\prime}\right)-M_{\psi_{k}}\left(\boldsymbol{u}_{k}, \boldsymbol{B}_{k}\right) \\
& \leq M_{\psi_{k}}\left(\boldsymbol{u}^{\prime}, \boldsymbol{B}^{\prime}\right)-M_{\psi}^{\prime}\left(\boldsymbol{u}^{\prime}, \boldsymbol{B}^{\prime}\right)+M_{\psi}^{\prime}\left(\boldsymbol{u}_{k}, \boldsymbol{B}_{k}\right)-M_{\psi_{k}}\left(\boldsymbol{u}_{k}, \boldsymbol{B}_{k}\right) \\
& \leq\left|M_{\psi}^{\prime}\left(\boldsymbol{u}^{\prime}, \boldsymbol{B}^{\prime}\right)-M_{\psi_{k}}\left(\boldsymbol{u}^{\prime}, \boldsymbol{B}^{\prime}\right)\right|+\left|M_{\psi}^{\prime}\left(\boldsymbol{u}_{k}, \boldsymbol{B}_{k}\right)-M_{\psi_{k}}\left(\boldsymbol{u}_{k}, \boldsymbol{B}_{k}\right)\right| \leq 2 \varepsilon^{\prime} .
\end{aligned}
$$

But from Lemma 12 and Lemma 13 we get that $\left\|\left(\begin{array}{c}\boldsymbol{B}^{\prime b} \\ \boldsymbol{u}^{\prime}\end{array}\right)-\left(\begin{array}{c}\boldsymbol{B}_{k}^{b} \\ \boldsymbol{u}_{k}\end{array}\right)\right\|_{2} \leq \frac{\varepsilon^{\prime}}{2}$. Now we can apply the Claim 2 which implies that

$$
\left\|\left(\begin{array}{c}
\left(\boldsymbol{\Sigma}^{*-1}\right)^{b} \\
\boldsymbol{\Sigma}^{*-1} \boldsymbol{\mu}^{*}
\end{array}\right)-\left(\begin{array}{c}
\boldsymbol{B}_{k}^{b} \\
\boldsymbol{u}_{k}
\end{array}\right)\right\|_{2} \leq \frac{\varepsilon^{\prime}}{2}
$$


Therefore it suffices to find $\left(\boldsymbol{u}_{k}, \boldsymbol{B}_{k}\right)$ with accuracy $\boldsymbol{\varepsilon}^{\prime} / 2$ to get our theorem.

Let $\boldsymbol{w}^{*}=\left(\begin{array}{l}\boldsymbol{B}_{k}^{b} \\ \boldsymbol{u}_{k}\end{array}\right)$ To prove that Algorithm 1 converges to $\boldsymbol{w}^{*}$ we use Theorem 5 which together with Markov's inequality, Lemma 11 and Lemma 12 gives us

$$
\mathbb{P}\left(M_{\psi_{k}}(\hat{\boldsymbol{u}}, \hat{\boldsymbol{B}})-M_{\psi_{k}}\left(\boldsymbol{u}_{k}, \boldsymbol{B}_{k}\right) \geq \operatorname{poly}\left(1 / \alpha^{*}\right) \cdot \frac{d^{2 k}}{T}(1+\log (T))\right) \leq \frac{1}{3} .
$$

To get our estimation we first repeat the SGD procedure $K=\log (1 / \delta)$ times independently, with parameters $T, \lambda$ each time. We then get the set of estimates $\mathcal{E}=\left\{\overline{\boldsymbol{w}}_{1}, \overline{\boldsymbol{w}}_{2}, \ldots, \overline{\boldsymbol{w}}_{K}\right\}$. Because of (12) we know that, with high probability $1-\delta$, for at least the $2 / 3$ of the points $\bar{w}$ in $\mathcal{E}$ it is true that $M_{\psi_{k}}(\boldsymbol{w})-M_{\psi_{k}}\left(\boldsymbol{w}^{*}\right) \leq \eta$ where $\eta=\operatorname{poly}\left(1 / \alpha^{*}\right) \cdot \frac{d^{2 k}}{T}(1+\log (T))$. Moreover we will prove later that $M_{\psi_{k}}(\boldsymbol{w})-M_{\psi_{k}}\left(\boldsymbol{w}^{*}\right) \leq \eta$ and this implies $\left\|\boldsymbol{w}-\boldsymbol{w}^{*}\right\| \leq c \cdot \eta$, where $c$ is a universal constant. Therefore with high probability $1-\delta$ for at least the $2 / 3$ of the points $\bar{w}, \bar{w}^{\prime}$ in $\mathcal{E}$ it is true that $\left\|w-w^{\prime}\right\| \leq 2 c \cdot \eta$. Hence if we set $\hat{w}$ to be a point that is at least $2 c \cdot \eta$ close to more that the half of the points in $\mathcal{E}$ then with high probability $1-\delta$ we have that $f(\overline{\boldsymbol{w}})-f\left(\boldsymbol{w}^{*}\right) \leq \eta$. Hence we can we lose probability at most $\delta$ if we condition on the event

$$
M_{\psi_{k}}(\hat{\boldsymbol{u}}, \hat{\boldsymbol{B}})-M_{\psi_{k}}\left(\boldsymbol{u}_{k}, \boldsymbol{B}_{k}\right) \leq \operatorname{poly}\left(1 / \alpha^{*}\right) \cdot \frac{d^{2 k}}{T}(1+\log (T)) .
$$

Using once again Lemma 13 we get that

$$
\left\|\left(\begin{array}{c}
\hat{\boldsymbol{B}}^{b} \\
\hat{\boldsymbol{u}}
\end{array}\right)-\left(\begin{array}{c}
\boldsymbol{B}_{k}^{b} \\
\boldsymbol{u}_{k}
\end{array}\right)\right\|_{2} \leq \frac{\varepsilon^{\prime}}{2} .
$$

which together with (11) implies

$$
\left\|\left(\begin{array}{c}
\hat{\boldsymbol{B}}^{b} \\
\hat{\boldsymbol{u}}
\end{array}\right)-\left(\begin{array}{c}
\left(\boldsymbol{\Sigma}^{*-1}\right)^{b} \\
\boldsymbol{\Sigma}^{*-1} \boldsymbol{\mu}^{*}
\end{array}\right)\right\|_{2} \leq \frac{\varepsilon^{\prime}}{2} .
$$

and the theorem follows as closeness in parameter distance implies closeness in total variation distance for the corresponding untruncated Gaussian distributions. 


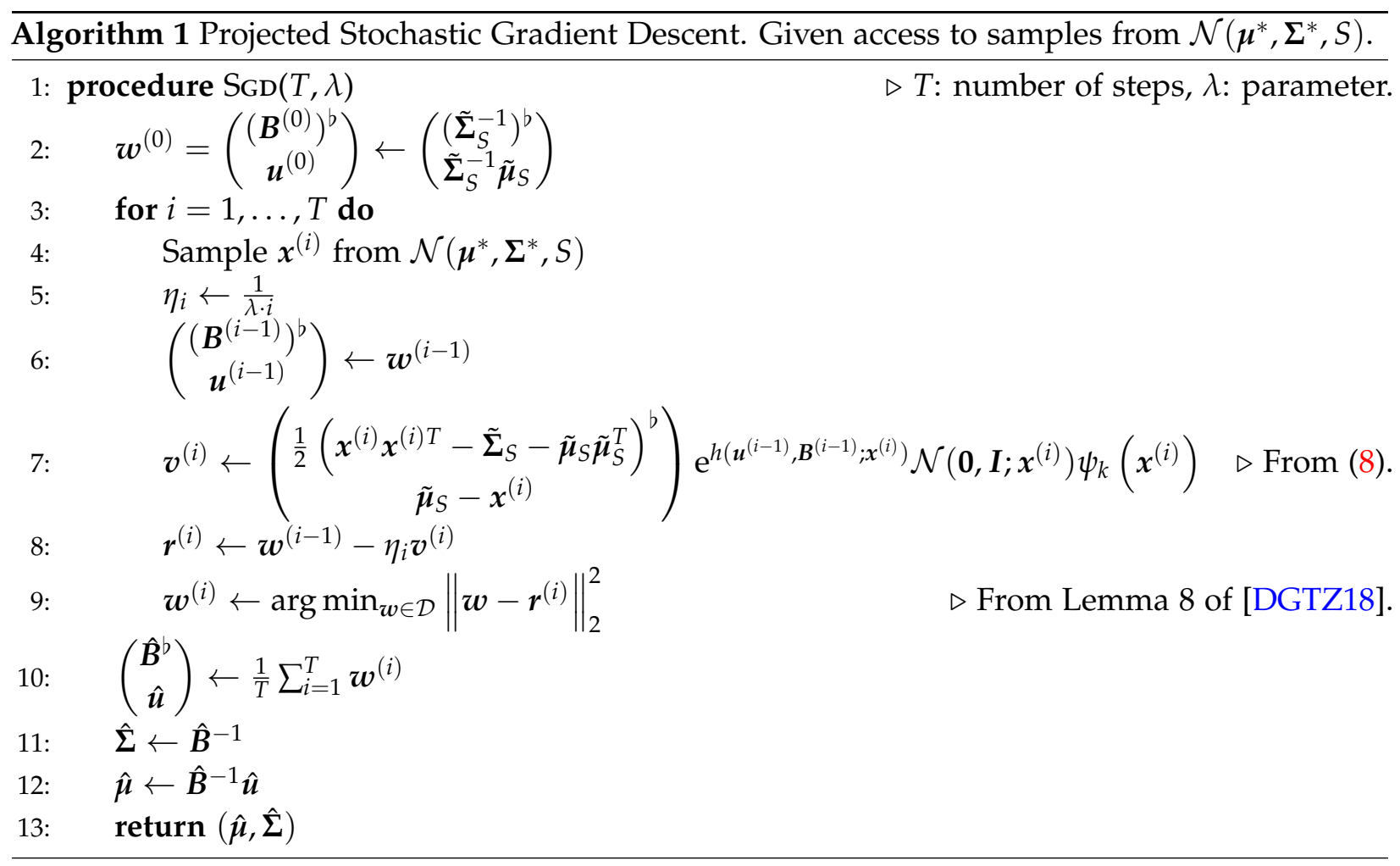

\subsection{Recovering the Set}

In this section we prove that, given only positive examples from an unknown truncated Gaussian distribution, that is samples from the conditional distribution on the truncation set, one can in fact learn the truncation set. We only give here the main result, for details see Appendix E.

Theorem 6 (RECOVERING THE SET). Let $\mathcal{S}$ be a class of measurable sets with Gaussian surface area at most $\Gamma(\mathcal{S})$. Let $\mathcal{N}^{*}$ be a Gaussian in $(O(\log (1 / \alpha), 1 / 16))$-isotropic position. Then, given $d^{\text {poly }}(1 / \alpha) \Gamma(\mathcal{S})^{2} / \varepsilon^{32}$ samples from the conditional distribution $\mathcal{N}_{S}^{*}$ we can recover an indicator of the set $\widetilde{S}$ such that with probability at least $99 \%$ it holds $\mathbb{P}_{x \sim \mathcal{N}^{*}}\left[\widetilde{S}(x) \neq \mathbf{1}_{S}(x)\right] \leq \varepsilon$.

\section{Lower Bound for Learning the Mean of a Truncated Normal}

Theorem 7. There exists a family of sets $\mathcal{S}$ with $\Gamma(\mathcal{S})=O(d)$ such that any algorithm that draws $m$ samples from $\mathcal{N}(\boldsymbol{\mu}, \boldsymbol{I}, S)$ and computes an estimate $\widetilde{\boldsymbol{\mu}}$ with $\|\widetilde{\boldsymbol{\mu}}-\boldsymbol{\mu}\|_{2} \leq 1$ must have $m=\Omega\left(2^{d / 2}\right)$.

Proof. Let $H=[-1,1]^{d+1}$ be the $d+1$-dimensional cube. We will also use the left and right subcubes $H_{+}=[-1,0] \times[-1,1]^{d}, H_{-}=[0,1] \times[-1,1]^{d}$ respectively. Let $\mathcal{N}_{+}=\mathcal{N}\left(\boldsymbol{e}_{1}, \boldsymbol{I}\right)$ and $\mathcal{N}_{-}=\mathcal{N}\left(-e_{1}, \boldsymbol{I}\right)$. We denote by $r$ the (scaled) pointwise minimum of the two densities truncated at the cube $H$, that is

$$
r(x)=\frac{\min \left(\mathcal{N}_{+}(H ; x), \mathcal{N}_{-}(H ; x)\right)}{c}=\frac{\mathbf{1}_{H}(x)}{c} \min \left(\mathcal{N}_{+}(x), \mathcal{N}_{-}(x)\right),
$$

where $c=1-d_{\mathrm{TV}}\left(\mathcal{N}_{+}, \mathcal{N}_{-}\right)$. 
To simplify notation we assume that we work in $\mathbb{R}^{d+1}$ instead of $\mathbb{R}^{d}$. Let $V=\left(v_{1}, \ldots, v_{d}\right) \in$ $\{+1,-1\}^{d}$. For every $V$ we define the set $G_{V}=H \cap\left\{\boldsymbol{y} \in \mathbb{R}^{d}: y_{i} v_{i} \geq 0\right\}$. We also define the subcubes $H_{V}=[0,1] \times G_{V}$. We consider the following subset of $H$ parameterized by the $2^{d}$ parameters $t_{V} \in[0,1]$ and $\delta \in[-1,1]$.

$$
S_{+}=[-1+\delta, 0] \times[-1,1]^{d} \cup \bigcup_{V \in\{-1,+1\}^{d}}\left[0, t_{V}\right] \times G_{V}
$$

We will argue that there exists a distribution $D^{+}$on the values $t_{V}$ such that on expectation $d_{\mathrm{TV}}\left(\mathcal{N}_{+}^{S_{+}}, \mathcal{N}_{-}^{S_{-}}\right)$is $O\left(2^{-d}\right)$. We show how to construct the distribution $D_{+}$since the construction for $D_{-}$is the same. In fact we will show that both distributions are very close to $r(x)$. Notice that for some $(t, y) \in \mathbb{R}^{d+1}$ we have We draw each $t_{V}$ independently from the distribution with cdf

$$
F(t)=\mathbf{1}_{[0,1)}(t)\left(1-\mathrm{e}^{-2 t}\right)+\mathbf{1}_{[1,+\infty)}(t)
$$

Notice that for $t \in(0,1)$ and any $\boldsymbol{y} \in \mathbb{R}^{d}$ we have that $1-F(t)=\mathcal{N}_{-}(t, \boldsymbol{y}) / \mathcal{N}_{+}(t, \boldsymbol{y})$.

After we draw all $t_{V}$ from $F$ we choose $\delta$ so that $\mathcal{N}_{+}\left(S_{+} ; x\right)=c$. We will show that on expectation over the $t_{V}$ we have $\delta=0$, which means that no correction is needed. In fact we show something stronger, namely that for all $x \in H_{+}$we have that $\mathbb{E}_{S_{+} \sim D_{+}}\left[N_{+}\left(S_{+} ; \boldsymbol{x}\right)\right]=r(\boldsymbol{x})$. Assume that $x \in H_{V}$. Indeed,

$$
\begin{aligned}
\underset{S_{+} \sim D_{+}}{\mathbb{E}}\left[\mathcal{N}_{+}\left(S_{+} ; \boldsymbol{x}\right)\right] & =\frac{\mathcal{N}_{+}(\boldsymbol{x})}{c} \underset{S_{+} \sim D_{+}}{\mathbb{E}}\left[\mathbf{1}_{S_{+}}(\boldsymbol{x})\right]=\frac{\mathcal{N}_{+}(\boldsymbol{x})}{c} \underset{S_{+} \sim D_{+}}{\mathbb{E}}\left[\mathbf{1}_{\left\{x_{1} \leq t_{V}\right\}}\right] \\
& =\frac{\mathcal{N}_{+}(\boldsymbol{x})}{c}\left(1-F\left(t_{V}\right)\right)=\frac{\mathcal{N}_{-}(\boldsymbol{x})}{c}=r(\boldsymbol{x})
\end{aligned}
$$

Moreover, observe that for all $\boldsymbol{x} \in H_{-} \cap S_{+}$we have that $N_{+}\left(S_{+} ; \boldsymbol{x}\right)=r(\boldsymbol{x})$ always (with probability $1)$. We now argue that in order to have constant probability to distinguish $N_{+}\left(S_{+}\right)$from $r(x)$ one needs to draw $\Omega\left(2^{d}\right)$ samples. Since the expected density of $N_{+}\left(S_{+}\right)$matches $r(x)$ for all $x \in H_{+}$, to be able to distinguish the two distributions one needs to observe at least two samples in the same cube $H_{V}$. Since we have $2^{d}$ disjoint cubes $H_{V}$ the probability of a sample landing in $H_{V}$ is at most $1 / 2^{d}$. Therefore, using the birthday problem, to have constant probability to observe a collision one needs to draw $\Omega\left(\sqrt{2^{d}}\right)=\Omega\left(2^{d / 2}\right)$ samples. Since for all $x \in H_{-} \cap S_{+}, N_{+}\left(S_{+}\right)$ exactly matches $r(x)$, to distinguish between the two distributions one needs to observe a sample $x$ with $-1+\delta<x_{1}<-1$. Due to symmetry, $N_{+}$assigns to all cubes $H_{V}$ equal probability, call that $p$. Moreover, we have that $c=2^{d+1} p$. Now let $p_{V}$ be the random variable corresponding to the probability that $N_{+}$assigns to $\left[0, t_{V}\right] \times G_{V}$. We have that $\mathbb{E}_{t_{V} \sim F}\left[p_{V}\right]=p$ for all $V$. Since the independent random variables $p_{V}$ are bounded in $\left[0,1 / 2^{d}\right]$, Hoeffding's inequality implies that $\left|\sum_{V \in\{-1,1\}^{d}}\left(p_{V}-p\right)\right|<1 / 2^{d / 2}$ with probability at least $1-2 / \mathrm{e}^{2}$. This means that with probability at least $3 / 4$ one will need to draw $\Omega\left(2^{d / 2}\right)$ samples in order to observe one with $x_{1}<-1+\delta$.

Since any set $S$ in our family $\mathcal{S}$ has almost everywhere (that is except the set of its vertices which a finite set and thus of measure zero) smooth boundary we may use the following equivalent (see e.g. [Naz03]) definition of its surface area

$$
\Gamma(S)=\int_{\partial S} \mathcal{N}_{0}(\boldsymbol{x}) \mathrm{d} \sigma(\boldsymbol{x}),
$$

where $\mathrm{d} \sigma(x)$ is the standard surface measure on $\mathbb{R}^{d}$. Without loss of generality we assume that $S$ 
Figure 2: The set $S_{+}$when $d=1$.

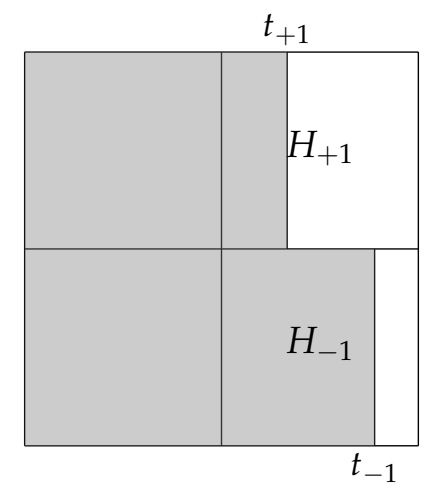

corresponds to the set $S_{+}$defined above (the proof is the same if we consider a set $S_{-}$). We have

$$
\partial S \subseteq \bigcup_{V \in\{+1,-1\}^{d}}\left(\left\{t_{V}\right\} \times G_{V}\right) \cup \partial\left([-1,+1]^{d+1}\right) \cup \bigcup_{i=1}^{d+1}\left\{x: x_{i}=0\right\} .
$$

By the definition of Gaussian surface area it is clear that $\Gamma(A \cup B) \leq \Gamma(A)+\Gamma(B)$. From Table 1 we know that $\Gamma\left([-1,+1]^{d+1}\right)=O(\sqrt{\log d})$. Moreover, we know that a single halfspace has surface area at most $\sqrt{2 / \pi}$ (see e.g. [KOS08]). Therefore $\Gamma\left(\bigcup_{i=1}^{d+1}\left\{x: x_{i}=0\right\}\right) \leq \sum_{i=1}^{d+1} \sqrt{2 / \pi}=O(d)$. Finally, we notice that for any point $x$ on the hyperplane $\left\{x: x_{1}=0\right\}$ and any $y$ on $\left\{x: x_{1}=c\right\}$ (for any $c \geq 0$ ), we have $\mathcal{N}_{0}(\boldsymbol{x}) \geq \mathcal{N}_{0}(\boldsymbol{y})$. Therefore, the surface area of each set $t_{V} \times G_{V}$ is maximized for $t_{V}=0$. In this case $\bigcup_{V \in\{+1,-1\}^{d}}\left(\left\{t_{V}\right\} \times G_{V}\right) \subseteq\left\{x: x_{1}=0\right\}$, which implies that the set $\bigcup_{V \in\{+1,-1\}^{d}}\left(\left\{t_{V}\right\} \times G_{V}\right)$ contributes at most $\sqrt{2 / \pi}$ to the total surface area. Putting everything together, we have that $\Gamma(S)=O(d)$.

\section{Identifiability with bounded Gaussian Surface Area}

In this section we investigate the sample complexity of the problem of estimating the parameters of a truncated Gaussian using a different approach that does not depend on the VC dimension of the family $\mathcal{S}$ of the truncation sets to be finite. For example, we settle the sample complexity of learning the parameters of a Gaussian distribution truncated at an unknown convex set (recall that the class of convex sets has infinite VC dimension). Our method relies on finding a tuple $(\widetilde{\mu}, \widetilde{\Sigma}, \widetilde{S})$ of parameters so that the moments of the corresponding truncated Gaussian $\mathcal{N}(\widetilde{\mu}, \widetilde{\Sigma}, \widetilde{S})$ are all close to the moments of the unknown truncated Gaussian distribution, for which we have unbiased estimates using samples. The main question that we need to answer to determine the sample complexity of this problem is how many moments are needed to be matched in order to be sure that our guessed parameters are close to the parameters of the unknown truncated Gaussian. We state now the main result. Its proof is based on Lemma 15 and can be found in Appendix F.

Theorem 8 (Moment Matching). Let $\mathcal{S}$ be a family of subsets of $\mathbb{R}^{d}$ of bounded Gaussian surface area $\Gamma(\mathcal{S})$. Moreover, assume that if $T$ is an affine map and $T(\mathcal{S})=\{T(S): S \in \mathcal{S}\}$ is the family of the images of the sets of $\mathcal{S}$, then it holds $\Gamma(T(\mathcal{S}))=O(\Gamma(\mathcal{S}))$. For some $S \in \mathcal{S}$, let $\mathcal{N}(\mu, \Sigma, S)$ be 
an unknown truncated Gaussian. $d^{O\left(\Gamma(\mathcal{S}) / \varepsilon^{4}\right)}$ samples are sufficient to find parameters $\widetilde{\mu}, \widetilde{\Sigma}, \widetilde{S}$ such that $d_{\mathrm{TV}}(\mathcal{N}(\boldsymbol{\mu}, \Sigma, S), \mathcal{N}(\widetilde{\mu}, \widetilde{\Sigma}, \widetilde{S})) \leq \varepsilon$.

The key lemma of this section is Lemma 15. It shows that if two truncated normals are in total variation distance $\varepsilon$ then there exists a moment where they differ. The main idea is to prove that there exists a polynomial that approximates well the indicator of the set $\left\{f_{1}>f_{2}\right\}$. Notice that the total variation distance between two densities can be written as $\int \mathbf{1}_{\left\{f_{1}>f_{2}\right\}}(\boldsymbol{x}) f_{1}(\boldsymbol{x})-f_{2}(\boldsymbol{x}) \mathrm{d} x$. In our proof we use the chi squared divergence, which for two distributions with densities $f_{1}, f_{2}$ is defined as

$$
D_{\chi^{2}}\left(f_{1} \| f_{2}\right)=\int \frac{\left(f_{1}(\boldsymbol{x})-f_{2}(\boldsymbol{x})\right)^{2}}{f_{2}(\boldsymbol{x})} \mathrm{d} x
$$

To prove it we need the following nice fact about chi squared divergence between Gaussian distributions. In general chi squared divergence may be infinite for some pairs of Gaussians. In the following lemma we prove that for any pair of Gaussians, there exists another Gaussian $N$ such that $D_{\chi^{2}}\left(N_{1} \| N\right) D_{\chi^{2}}\left(N_{2} \| N\right)$ are finite even if $D_{\chi^{2}}\left(N_{1} \| N_{2}\right)=\infty$.

Lemma 14. Let $N_{1}=\mathcal{N}\left(\boldsymbol{\mu}_{1}, \boldsymbol{\Sigma}_{1}\right)$, and $N_{2}=\mathcal{N}\left(\boldsymbol{\mu}_{1}, \boldsymbol{\Sigma}_{2}\right)$ be two Normal distributions that satisfy the conditions of Lemma 18. Then there exists a Normal distribution $N$ such that

$$
D_{\chi^{2}}\left(N_{1} \| N\right), D_{\chi^{2}}\left(N_{2} \| N\right) \leq \exp \left(2\left\|\Sigma_{1}^{-1 / 2}\left(\boldsymbol{\mu}_{1}-\boldsymbol{\mu}_{2}\right)\right\|_{2}+\frac{1}{2} \max \left(1,\left\|\boldsymbol{\Sigma}_{1}\right\|_{2}\right)\left\|\boldsymbol{\Sigma}_{1}^{-1 / 2} \boldsymbol{\Sigma}_{2} \boldsymbol{\Sigma}_{1}^{-1 / 2}-\boldsymbol{I}\right\|_{F}^{2}\right)
$$

Now we state the main lemma of this section. We give here a sketch of its proof. It's full version can be found in Appendix F.

Lemma 15. Let $\mathcal{S}$ be a family of subsets of $\mathbb{R}^{d}$ of bounded Gaussian surface area $\Gamma(\mathcal{S})$. Moreover, assume that if $T$ is an affine map and $T(\mathcal{S})=\{T(S): S \in \mathcal{S}\}$ is the family of the images of the sets of $\mathcal{S}$, then it holds $\Gamma(T(\mathcal{S}))=O(\Gamma(\mathcal{S}))$. Let $\mathcal{N}\left(\boldsymbol{\mu}_{1}, \boldsymbol{\Sigma}_{1}, S_{1}\right)$ and $\mathcal{N}\left(\boldsymbol{\mu}_{2}, \boldsymbol{\Sigma}_{2}, S_{2}\right)$ be two truncated Gaussians with densities $f_{1}, f_{2}$ respectively. Let $k=O\left(\Gamma(\mathcal{S}) / \varepsilon^{4}\right)$. If $d_{\mathrm{TV}}\left(f_{1}, f_{2}\right) \geq \varepsilon$, then there exists a $V \in \mathbb{N}^{d}$ with $|V| \leq k$ such that

$$
\left|\underset{x \sim \mathcal{N}\left(\mu_{1}, \Sigma_{1}, S_{1}\right)}{\mathbb{E}}\left[x^{V}\right]-\underset{x \sim \mathcal{N}\left(\mu_{2}, \Sigma_{2}, S_{2}\right)}{\mathbb{E}}\left[x^{V}\right]\right| \geq \varepsilon / d^{O(k)} .
$$

Proof sketch. Let $W=S_{1} \cap S_{2} \cap\left\{f_{1}>f_{2}\right\} \cup S_{1} \backslash S_{2}$, that is the set of points where the first density is larger than the second. We now write the $L_{1}$ distance between $f_{1}, f_{2}$ as

$$
\int\left|f_{1}(\boldsymbol{x})-f_{2}(\boldsymbol{x})\right| \mathrm{d} \boldsymbol{x}=\int \mathbf{1}_{W}(\boldsymbol{x})\left(f_{1}(\boldsymbol{x})-f_{2}(\boldsymbol{x})\right) \mathrm{d} \boldsymbol{x}
$$

Denote $p(x)$ the polynomial that will do the approximation of the $L_{1}$ distance. From Lemma 14 we know that there exists a Normal distribution within small chi-squared divergence of both $\mathcal{N}\left(\boldsymbol{\mu}_{1}, \boldsymbol{\Sigma}_{1}\right)$ and $\mathcal{N}\left(\boldsymbol{\mu}_{2}, \boldsymbol{\Sigma}_{2}\right)$. Call the density function of this distribution $g(\boldsymbol{x})$. We have

$$
\begin{aligned}
\left|\int\right| f_{1}(\boldsymbol{x}) & -f_{2}(\boldsymbol{x})\left|\mathrm{d} \boldsymbol{x}-\int p(\boldsymbol{x})\left(f_{1}(\boldsymbol{x})-f_{2}(\boldsymbol{x})\right)\right| \\
& \leq \int\left|\mathbf{1}_{W}(\boldsymbol{x})-p(\boldsymbol{x})\right|\left|f_{1}(\boldsymbol{x})-f_{2}(\boldsymbol{x})\right| \mathrm{d} \boldsymbol{x} \\
& \leq \int\left|\mathbf{1}_{W}(\boldsymbol{x})-p(\boldsymbol{x})\right| \sqrt{g(\boldsymbol{x})} \frac{\left|f_{1}(\boldsymbol{x})-f_{2}(\boldsymbol{x})\right|}{\sqrt{g(\boldsymbol{x})}} \mathrm{d} x \\
& \leq \sqrt{\int\left(\mathbf{1}_{W}(\boldsymbol{x})-p(\boldsymbol{x})\right)^{2} g(\boldsymbol{x}) \mathrm{d} \boldsymbol{x}} \sqrt{\int \frac{\left(f_{1}(\boldsymbol{x})-f_{2}(\boldsymbol{x})\right)^{2}}{g(\boldsymbol{x})}} \mathrm{d} \boldsymbol{x},
\end{aligned}
$$


where we use Schwarzs' inequality. From Lemma 14 we know that

$$
\int \frac{f_{1}(\boldsymbol{x})^{2}}{g(\boldsymbol{x})} \mathrm{d} x \leq \int \frac{\mathcal{N}\left(\boldsymbol{\mu}_{1}, \boldsymbol{\Sigma}_{1} ; \boldsymbol{x}\right)^{2}}{g(\boldsymbol{x})} \mathrm{d} x=\exp (\operatorname{poly}(1 / \alpha)) .
$$

Similarly, $\int \frac{f_{2}(x)^{2}}{g(x)} \mathrm{d} x=\exp (\operatorname{poly}(1 / \alpha))$. Therefore we have,

$$
\left|\int\right| f_{1}(\boldsymbol{x})-f_{2}(\boldsymbol{x})\left|\mathrm{d} \boldsymbol{x}-\int p(\boldsymbol{x})\left(f_{1}(\boldsymbol{x})-f_{2}(\boldsymbol{x})\right)\right| \leq \exp (\operatorname{poly}(1 / \alpha)) \sqrt{\int\left(\mathbf{1}_{W}(\boldsymbol{x})-p(\boldsymbol{x})\right)^{2} g(\boldsymbol{x}) \mathrm{d} \boldsymbol{x}}
$$

Recall that $g(\boldsymbol{x})$ is the density function of a Gaussian distribution, and let $\mu, \Sigma$ be the parameters of this Gaussian. Notice that it remains to show that there exists a good approximating polynomial $p(x)$ to the indicator function $\mathbf{1}_{W}$. We can now transform the space so that $g(x)$ becomes the standard normal. Notice that this is an affine transformation that also transforms the set $W$; Since the Gaussian surface area is "invariant" under linear transformations

Since $\mathbf{1}_{W} \in L^{2}\left(\mathbb{R}^{d}, \mathcal{N}_{0}\right)$ we can approximate it using Hermite polynomials. For some $k \in \mathcal{N}$ we set $p(\boldsymbol{x})=S_{k} \mathbf{1}_{W}(x)$, that is

$$
p_{k}(\boldsymbol{x})=\sum_{V:|V| \leq k} \widehat{\mathbf{1}_{W}} H_{V}(\boldsymbol{x})
$$

Combining Lemma 5 and Lemma 19 we obtain

$$
\underset{x \sim \mathcal{N}_{0}}{\mathbb{E}}\left[\left(\mathbf{1}_{W}(\boldsymbol{x})-p_{k}(x)\right)^{2}\right]=O\left(\frac{\Gamma(\mathcal{S})}{k^{1 / 2}}\right) .
$$

Therefore, $\left|\int\right| f_{1}(\boldsymbol{x})-f_{2}(\boldsymbol{x})\left|\mathrm{d} \boldsymbol{x}-\int p_{k}(\boldsymbol{x})\left(f_{1}(\boldsymbol{x})-f_{2}(\boldsymbol{x})\right)\right|=\exp (\operatorname{poly}(1 / \alpha)) \frac{\Gamma(\mathcal{S})^{1 / 2}}{k^{1 / 4}}$. Ignoring the dependence on the absolute constant $\alpha$, to achieve error $O(\varepsilon)$ we need degree $k=O\left(\Gamma(\mathcal{S})^{2} / \varepsilon^{4}\right)$.

To complete the proof, it remains to obtain a bound for the coefficients of the polynomial $q(\boldsymbol{x})=p_{k}\left(\boldsymbol{\Sigma}^{-1 / 2}(\boldsymbol{x}-\boldsymbol{\mu})\right)$. Using known facts about the coefficients of Hermite polynomials we obtain that $\|q(x)\|_{\infty} \leq\left(\begin{array}{c}d+k \\ k\end{array}\right)^{2}(4 d)^{k / 2}\left(O\left(1 / \alpha^{2}\right)\right)^{k}$. To conclude the proof we notice that we can pick the degree $k$ so that

$$
\left|\int q(\boldsymbol{x})\left(f_{1}(\boldsymbol{x})-f_{2}(\boldsymbol{x})\right)\right|=\left|\sum_{V:|V| \leq k} x^{V}\left(f_{1}(\boldsymbol{x})-f_{2}(\boldsymbol{x})\right)\right| \geq \varepsilon / 2 .
$$

Since the maximum coefficient of $q(\boldsymbol{x})$ is bounded by $d^{O(k)}$ we obtain the result.

\section{VC-dimension vs Gaussian Surface Area}

We use two different complexity measures of the truncation set to get sample complexity bounds, the VC-dimension and the Gaussian Surface Area (GSA) of the class of the sets. As we already mentioned in the introduction, there are classes, for example convex sets, that have bounded Gaussian surface area but infinite VC-dimension. However, this is not the main difference between the two complexity measures in our setting. Having a class with bounded VC-dimension means that the empirical risk minimization needs finite samples. To get an efficient algorithm we still need to implement the ERM for this specific class. Therefore, it is not clear whether it is possible to get an algorithm that works for all sets of bounded VC-dimension. On the other hand, bounded 
GSA means that we can approximate the weighted indicator of the set using its low order Hermite coeffients. This approximation works for all sets of bounded GSA and does not depend on the specific class of sets. Therefore, using GSA we manage to get a unified approach that learns the parameters of the underlying Gaussian distribution using only the assumption that the truncation set has bounded GSA. In other words, our approach uses the information of the class that the truncation set belongs only to decide how large the degree of the approximating polynomial should be. Having said that, it is an interesting open problem to design algorithms that learn the parameters of the Gaussian and use the information that the truncation set belongs to some class (e.g. intersection of $k$-halfspaces) to beat the runtime of our generic approach that only depends on the GSA of the class. 


\section{References}

[AGR13] Joseph Anderson, Navin Goyal, and Luis Rademacher. Efficient learning of simplices. In Conference on Learning Theory, pages 1020-1045, 2013.

[Ba193] Keith Ball. The reverse isoperimetric problem for gaussian measure. Discrete $\mathcal{E}$ Computational Geometry, 10(1):411-420, 1993.

[BC14] N Balakrishnan and Erhard Cramer. The art of progressive censoring. Springer, 2014.

[Coh16] A Clifford Cohen. Truncated and censored samples: theory and applications. CRC press, 2016.

[CSV17] Moses Charikar, Jacob Steinhardt, and Gregory Valiant. Learning from untrusted data. In Proceedings of the 49th Annual ACM SIGACT Symposium on Theory of Computing, STOC 2017, Montreal, QC, Canada, June 19-23, 2017, pages 47-60, 2017.

[CW01] Anthony Carbery and James Wright. Distributional and lâ norm inequalities for polynomials over convex bodies in rn. Mathematical research letters, 8(3):233-248, 2001.

[DDS14] Anindya De, Ilias Diakonikolas, and Rocco A Servedio. Learning from satisfying assignments. In Proceedings of the twenty-sixth annual ACM-SIAM symposium on Discrete algorithms, pages 478-497. SIAM, 2014.

[Den98] François Denis. Pac learning from positive statistical queries. In International Conference on Algorithmic Learning Theory, pages 112-126. Springer, 1998.

[DGTZ18] Constantinos Daskalakis, Themis Gouleakis, Christos Tzamos, and Manolis Zampetakis. Efficient statistics, in high dimensions, from truncated samples. In the 59th Annual IEEE Symposium on Foundations of Computer Science (FOCS), 2018.

[DK14] Constantinos Daskalakis and Gautam Kamath. Faster and sample near-optimal algorithms for proper learning mixtures of gaussians. In Proceedings of The 27th Conference on Learning Theory, COLT 2014, Barcelona, Spain, June 13-15, 2014, pages 1183-1213, 2014.

[DKK $\left.{ }^{+} 16\right]$ Ilias Diakonikolas, Gautam Kamath, Daniel M. Kane, Jerry Li, Ankur Moitra, and Alistair Stewart. Robust estimators in high dimensions without the computational intractability. In IEEE 57th Annual Symposium on Foundations of Computer Science, FOCS 2016, 9-11 October 2016, Hyatt Regency, New Brunswick, New Jersey, USA, pages 655-664, 2016.

[DKK ${ }^{+}$17] Ilias Diakonikolas, Gautam Kamath, Daniel M. Kane, Jerry Li, Ankur Moitra, and Alistair Stewart. Being robust (in high dimensions) can be practical. In Proceedings of the 34th International Conference on Machine Learning, ICML 2017, Sydney, NSW, Australia, 6-11 August 2017, pages 999-1008, 2017.

[DKK $\left.{ }^{+} 18\right]$ Ilias Diakonikolas, Gautam Kamath, Daniel M. Kane, Jerry Li, Ankur Moitra, and Alistair Stewart. Robustly learning a gaussian: Getting optimal error, efficiently. In Proceedings of the Twenty-Ninth Annual ACM-SIAM Symposium on Discrete Algorithms, SODA 2018, New Orleans, LA, USA, January 7-10, 2018, pages 2683-2702, 2018. 
[DL12] Luc Devroye and Gábor Lugosi. Combinatorial methods in density estimation. Springer Science \& Business Media, 2012.

[Eld11] Ronen Eldan. A polynomial number of random points does not determine the volume of a convex body. Discrete E Computational Geometry, 46(1):29-47, 2011.

[Fis31] RA Fisher. Properties and applications of Hh functions. Mathematical tables, 1:815-852, 1931.

[FJK96] Alan Frieze, Mark Jerrum, and Ravi Kannan. Learning linear transformations. In Foundations of Computer Science, 1996. Proceedings., 37th Annual Symposium on, pages 359-368. IEEE, 1996.

[Ga197] Francis Galton. An examination into the registered speeds of american trotting horses, with remarks on their value as hereditary data. Proceedings of the Royal Society of London, 62(379-387):310-315, 1897.

[GR09] Navin Goyal and Luis Rademacher. Learning convex bodies is hard. arXiv preprint arXiv:0904.1227, 2009.

[Kan11] Daniel M Kane. The gaussian surface area and noise sensitivity of degree-d polynomial threshold functions. computational complexity, 20(2):389-412, 2011.

[KKMS05] Adam Tauman Kalai, Adam R. Klivans, Yishay Mansour, and Rocco A. Servedio. Agnostically learning halfspaces. In 46th Annual IEEE Symposium on Foundations of Computer Science (FOCS 2005), 23-25 October 2005, Pittsburgh, PA, USA, Proceedings, pages 11-20, 2005.

[KOS08] Adam R. Klivans, Ryan O'Donnell, and Rocco A. Servedio. Learning geometric concepts via gaussian surface area. In 49th Annual IEEE Symposium on Foundations of Computer Science, FOCS 2008, October 25-28, 2008, Philadelphia, PA, USA, pages 541-550, 2008.

[LDG00] Fabien Letouzey, François Denis, and Rémi Gilleron. Learning from positive and unlabeled examples. In International Conference on Algorithmic Learning Theory, pages 71-85. Springer, 2000.

[Led94] Michel Ledoux. Semigroup proofs of the isoperimetric inequality in euclidean and gauss space. Bulletin des sciences mathématiques, 118(6):485-510, 1994.

[Lee14] Alice Lee. Table of the gaussian" tail" functions; when the" tail" is larger than the body. Biometrika, 10(2/3):208-214, 1914.

[LRV16] Kevin A. Lai, Anup B. Rao, and Santosh Vempala. Agnostic estimation of mean and covariance. In IEEE 57th Annual Symposium on Foundations of Computer Science, FOCS 2016, 9-11 October 2016, Hyatt Regency, New Brunswick, New Jersey, USA, pages 665-674, 2016.

[Naz03] Fedor Nazarov. On the Maximal Perimeter of a Convex Set in $\mathbb{R}^{n}$ with Respect to a Gaussian Measure, pages 169-187. Springer Berlin Heidelberg, Berlin, Heidelberg, 2003. 
[O'D14] Ryan O'Donnell. Analysis of Boolean Functions. Cambridge University Press, 2014.

[Pea02] Karl Pearson. On the systematic fitting of frequency curves. Biometrika, 2:2-7, 1902.

[Pis86] Gilles Pisier. Probabilistic methods in the geometry of banach spaces. In Probability and analysis, pages 167-241. Springer, 1986.

[PL08] Karl Pearson and Alice Lee. On the generalised probable error in multiple normal correlation. Biometrika, 6(1):59-68, 1908.

[Sch86] Helmut Schneider. Truncated and censored samples from normal populations. Marcel Dekker, Inc., 1986.

[SJ66] SM Shah and MC Jaiswal. Estimation of parameters of doubly truncated normal distribution from first four sample moments. Annals of the Institute of Statistical Mathematics, 18(1):107-111, 1966.

[SSBD14] Shai Shalev-Shwartz and Shai Ben-David. Understanding machine learning: From theory to algorithms. Cambridge university press, 2014.

[Sze67] G. Szegö. Orthogonal Polynomials. Number $\tau .23$ in American Mathematical Society colloquium publications. American Mathematical Society, 1967.

\section{A Additional Preliminaries and Notation}

We first state the following simple lemma that connects the total variation distance of two Normal distributions with their parameter distance. For a proof see e.g. Corollaries 2.13 and 2.14 of $\left[\mathrm{DKK}^{+} 16\right]$.

Lemma 16. Let $N_{1}=\mathcal{N}\left(\boldsymbol{\mu}_{1}, \boldsymbol{\Sigma}_{1}\right), N_{2}=\mathcal{N}\left(\boldsymbol{\mu}_{2}, \boldsymbol{\Sigma}_{2}\right)$ be two Normal distributions. Then

$$
d_{\mathrm{TV}}\left(N_{1}, N_{2}\right) \leq \frac{1}{2}\left\|\boldsymbol{\Sigma}_{1}^{-1 / 2}\left(\boldsymbol{\mu}_{1}-\boldsymbol{\mu}_{2}\right)\right\|_{2}+\sqrt{2}\left\|\boldsymbol{I}-\boldsymbol{\Sigma}_{1}^{-1 / 2} \boldsymbol{\Sigma}_{2} \boldsymbol{\Sigma}_{1}^{-1 / 2}\right\|_{F}
$$

We readily use the following two lemmas from [DGTZ18]. The first suggests that we can accurately estimate the parameters $\left(\mu_{S}, \Sigma_{S}\right)$.

Lemma 17. Let $\left(\boldsymbol{\mu}_{S}, \boldsymbol{\Sigma}_{S}\right)$ be the mean and covariance of the truncated Gaussian $\mathcal{N}(\boldsymbol{\mu}, \boldsymbol{\Sigma}, S)$ for a set $S$ such that $\mathcal{N}(\mu, \Sigma ; S) \geq \alpha$. Using $\widetilde{O}\left(\frac{d}{\varepsilon^{2}} \log (1 / \alpha) \log ^{2}(1 / \delta)\right)$ samples, we can compute estimates $\widetilde{\mu}_{S}$ and $\widetilde{\Sigma}_{S}$ such that with probability at least $1-\delta$,

$$
\left\|\boldsymbol{\Sigma}^{-1 / 2}\left(\widetilde{\boldsymbol{\mu}}_{S}-\boldsymbol{\mu}_{S}\right)\right\|_{2} \leq \varepsilon \quad \text { and } \quad(1-\varepsilon) \boldsymbol{\Sigma}_{S} \preceq \widetilde{\boldsymbol{\Sigma}}_{S} \preceq(1+\varepsilon) \boldsymbol{\Sigma}_{S}
$$

The second lemma suggests that the empirical estimates are close to the true parameters of underlying truncated Gaussian.

Lemma 18. The empirical mean and covariance $\widetilde{\boldsymbol{\mu}}_{S}$ and $\widetilde{\boldsymbol{\Sigma}}_{S}$ computed using $\tilde{O}\left(d^{2} \log ^{2}(1 / \alpha \delta)\right)$ samples from a truncated Normal $\mathcal{N}(\mu, \Sigma, S)$ with $\mathcal{N}(\mu, \Sigma ; S) \geq \alpha$ satisfies with probability $1-\delta$ that:

$$
\left\|\Sigma^{-1 / 2}\left(\widetilde{\boldsymbol{\mu}}_{S}-\boldsymbol{\mu}\right)\right\|_{2}^{2} \leq O\left(\log \frac{1}{\alpha}\right), \quad \widetilde{\boldsymbol{\Sigma}}_{S} \succeq \Omega\left(\alpha^{2}\right) \Sigma, \quad\left\|\Sigma^{-1 / 2} \widetilde{\boldsymbol{\Sigma}}_{S} \Sigma^{-1 / 2}-\boldsymbol{I}\right\|_{F}^{2} \leq O\left(\log \frac{1}{\alpha}\right) .
$$

Moreover, $\Omega\left(\alpha^{2}\right) \leq\left\|\widetilde{\Sigma}_{S}^{-1 / 2} \Sigma \widetilde{\Sigma}_{S}^{-1 / 2}\right\|_{2} \leq O\left(1 / \alpha^{2}\right)$. 
In particular, the mean and covariance $\widetilde{\mu}_{S}$ and $\widetilde{\boldsymbol{\Sigma}}_{S}$ that satisfy the conditions of Lemma 18 , are in $\left(O(\log (1 / \alpha)), 1-O\left(\alpha^{2}\right)\right)$-near isotropic position.

We will use the following very useful anti-concentration result about the Gaussian mass of sets defined by polynomials.

Theorem 9 (Theorem 8 of [CW01]). Let $q, \gamma \in \mathbb{R}_{+}, \mu \in \mathbb{R}^{d}, \boldsymbol{\Sigma} \in \mathbb{R}^{d \times d}$ such that $\boldsymbol{\Sigma}$ is symmetric positive semidefinite and $p: \mathbb{R}^{d} \rightarrow \mathbb{R}$ be a multivariate polynomial of degree at most $\ell$, we define

$$
\bar{Q}=\left\{x \in \mathbb{R}^{d}|| p(x) \mid \leq \gamma\right\}
$$

then there exists an absolute constant $C$ such that

$$
\mathcal{N}(\mu, \Sigma ; \bar{Q}) \leq \frac{C q \gamma^{1 / \ell}}{\left(\mathbb{E}_{z \sim \mathcal{N}(\mu, \Sigma)}\left[|p(z)|^{q / \ell}\right]\right)^{1 / q}}
$$

\section{A.1 Hermite Polynomials, Ornstein-Uhlenbeck Operator, and Gaussian Surface Area.}

We denote by $L^{2}\left(\mathbb{R}^{d}, \mathcal{N}_{0}\right)$ the vector space of all functions $f: \mathbb{R}^{d} \rightarrow \mathbb{R}$ such that $\mathbb{E}_{x \sim \mathcal{N}_{0}}\left[f^{2}(x)\right]<\infty$. The usual inner product for this space is $\mathbb{E}_{\boldsymbol{x} \sim \mathcal{N}_{0}}[f(\boldsymbol{x}) g(\boldsymbol{x})]$. While, usually one considers the probabilists's or physicists' Hermite polynomials, in this work we define the normalized Hermite polynomial of degree $i$ to be $H_{0}(x)=1, H_{1}(x)=x, H_{2}(x)=\frac{x^{2}-1}{\sqrt{2}}, \ldots, H_{i}(x)=\frac{H e_{i}(x)}{\sqrt{i} !}, \ldots$ where by $H e_{i}(x)$ we denote the probabilists' Hermite polynomial of degree $i$. These normalized Hermite polynomials form a complete orthonormal basis for the single dimensional version of the inner product space defined above. To get an orthonormal basis for $L^{2}\left(\mathbb{R}^{d}, \mathcal{N}_{0}\right)$, we use a multi-index $V \in \mathbb{N}^{d}$ to define the $d$-variate normalized Hermite polynomial as $H_{V}(\boldsymbol{x})=\prod_{i=1}^{d} H_{v_{i}}\left(x_{i}\right)$. The total degree of $H_{V}$ is $|V|=\sum v_{i} \in V v_{i}$. Given a function $f \in L^{2}$ we compute its Hermite coefficients as $\hat{f}(V)=\mathbb{E}_{\boldsymbol{x} \sim \mathcal{N}_{0}}\left[f(\boldsymbol{x}) H_{V}(\boldsymbol{x})\right]$ and express it uniquely as $\sum_{V \in \mathbb{N}^{d}} \hat{f}(V) H_{V}(\boldsymbol{x})$. We denote by $S_{k} f(x)$ the degree $k$ partial sum of the Hermite expansion of $f, S_{k} f(\boldsymbol{x})=\sum_{|V| \leq k} \hat{f}(V) H_{V}(\boldsymbol{x})$. Then, since the basis of Hermite polynomials is complete, we have $\lim _{k \rightarrow \infty} \mathbb{E}_{x \sim \mathcal{N}_{0}}\left[\left(f(x)-S_{k} f(x)\right)^{2}\right]=0$. We would like to quantify the convergence rate of $S_{k} f$ to $f$. Parseval's identity states that

$$
\underset{x \sim \mathcal{N}_{0}}{\mathbb{E}}\left[\left(f(\boldsymbol{x})-S_{k} f(\boldsymbol{x})\right)^{2}\right]=\sum_{|V|=k}^{\infty} \hat{f}(V)^{2} .
$$

Definition 3 (Hermite Concentration). Let $\gamma(\varepsilon, d)$ be a function $\gamma:(0,1 / 2) \times \mathbb{N} \mapsto \mathbb{N}$. We say that a class of functions $\mathcal{F}$ over $\mathbb{R}^{d}$ has a Hermite concentration bound of $\gamma(\varepsilon, d)$, if for all $d \geq 1$, all $\varepsilon \in(0,1 / 2)$, and $f \in \mathcal{F}$ it holds $\sum_{|V| \geq \gamma(\varepsilon, d)} \hat{f}(V)^{2} \leq \varepsilon$.

We now define the Gaussian Noise Operator as in [O'D14]. Using a different parametrization, which is not convenient for our purposes, these operators are also known as the OrnsteinUhlenbeck semigroup, or the Mehler transform.

Definition 4. The Gaussian Noise operator $T_{\rho}$ is the linear operator defined on the space of functions $L^{1}\left(\mathbb{R}^{d}, \mathcal{N}_{0}\right)$ by

$$
T_{\rho} f(\boldsymbol{x})=\underset{\boldsymbol{y} \sim \mathcal{N}_{0}}{\mathbb{E}}\left[f\left(\rho \boldsymbol{x}+\sqrt{1-\rho^{2}} \boldsymbol{y}\right)\right] .
$$


A nice property of operator $T_{1-\rho}$ that we will use is that it has a simple Hermite expansion

$$
S_{k}\left(T_{\rho} f\right)(\boldsymbol{x})=\sum_{V:|V| \leq k} \rho^{|V|} \widehat{f}(V) H_{V}(\boldsymbol{x})
$$

We also define the noise sensitivity of a function $f$.

Definition 5 (Noise Sensitivity). Let $f: \mathbb{R}^{d} \mapsto \mathbb{R}$ be a function in $L^{2}\left(\mathbb{R}^{d}, \mathcal{N}_{0}\right)$. The noise sensitivity of $f$ at $\rho \in[0,1]$ is defined to be

$$
\mathbf{N S}_{\rho}[f]=2 \underset{x \sim \mathcal{N}_{0}}{\mathbb{E}}\left[f(x)^{2}-f(x) T_{1-\rho} f(x)\right]
$$

Since, the vectors $x$ and $z=(1-\rho) x+\sqrt{1-\rho^{2}} y$ are jointly distributed according to

$$
D_{\rho}=\mathcal{N}\left(\left(\begin{array}{l}
\mathbf{0} \\
\mathbf{0}
\end{array}\right),\left(\begin{array}{cc}
\boldsymbol{I} & (1-\rho) \boldsymbol{I} \\
(1-\rho) \boldsymbol{I} & \boldsymbol{I}
\end{array}\right)\right) .
$$

we can write

$$
\mathbf{N S}_{\rho}[f]=\underset{(x, z) \sim D_{\rho}}{\mathbb{E}}\left[f(\boldsymbol{x})^{2}\right]+\underset{(x, z) \sim D_{\rho}}{\mathbb{E}}\left[f(\boldsymbol{z})^{2}-2 f(\boldsymbol{x}) f(\boldsymbol{z})\right]=\underset{(x, \boldsymbol{z}) \sim D_{\rho}}{\mathbb{E}}\left[(f(\boldsymbol{x})-f(\boldsymbol{z}))^{2}\right] .
$$

When $f$ is an indicator of a set, the noise sensitivity is

$$
\mathbf{N S}_{\rho}\left[\mathbf{1}_{S}\right]=2 \underset{(x, z)}{\mathbb{E}}\left[\mathbf{1}_{S}(x)\left(1-\mathbf{1}_{S}(\boldsymbol{z})\right)\right]=2 \underset{(x, z)}{\mathbb{E}}\left[\mathbf{1}_{S}(x) \mathbf{1}_{S^{c}}(\boldsymbol{z})\right]
$$

which is equal to the probability of the correlated points $x, z$ landing at "opposite" sides of $S$.

Ledoux [Led94] and Pisier [Pis86] showed that the noise sensitivity of a set can be bounded by its Gaussian surface area.

Definition 6 (Gaussian Surface Area). For a Borel set $A \subseteq \mathbb{R}^{d}$, its Gaussian surface area is $\Gamma(A)=$ $\liminf _{\delta \rightarrow 0} \frac{\mathcal{N}_{0}\left(A_{\delta} \backslash A\right)}{\delta}$, where $A_{\delta}=\{x: \operatorname{dist}(x, A) \leq \delta\}$.

We will use the following lemma given in [KOS08].

Lemma 19 (Corollary 14 of [KOS08]). For a Borel set $S \subseteq \mathbb{R}^{d}$ and $\rho \geq 0, \mathbf{N S}_{\rho}\left[\mathbf{1}_{S}(\boldsymbol{x})\right] \leq \sqrt{\pi} \sqrt{\rho} \Gamma(S)$.

For more details on the Gaussian space and Hermite Analysis (especially from the theoretical computer science perspective), we refer the reader to [O'D14]. Most of the facts about Hermite polynomials that we shall use in this work are well known properties and can be found, for example, in [Sze67].

\section{B Missing proofs of Section 3}

We will use a standard tournament based approach for selecting a good hypotheses. We will use a version of the tournament from [DK14]. See also [DL12].

Lemma 20 (Tournament [DK14]). There is an algorithm, which is given sample access to some distribution $X$ and a collection of distributions $\mathcal{H}=\left\{H_{1}, \ldots, H_{N}\right\}$ over some set, access to a PDF comparator for every pair of distributions $H_{i}, H_{j} \in \mathcal{H}$, an accuracy parameter $\varepsilon>0$, and a confidence parameter $\delta>0$. The algorithm makes $\left.O\left(\log (1 / \delta) \varepsilon^{2}\right) \log N\right)$ draws from each of $X, H_{1}, \ldots, H_{N}$ and returns some $H \in \mathcal{H}$ or declares "failure" If there is some $H \in \mathcal{H}$ such that $d_{\mathrm{TV}}(H, X) \leq \varepsilon$ then with probability at least $1-\delta$ the returned distribution $H$ satisfies $d_{\mathrm{TV}}(H, X) \leq 512 \varepsilon$. The total number of operations of the algorithm is $O\left(\log (1 / \delta)\left(1 / \varepsilon^{2}\right)(N \log N+\log 1 / \delta)\right)$. 
We first argue that if the class of sets $\mathcal{S}$ has $\mathrm{VC}$-dimension $\operatorname{VC}(\mathcal{S})$ then we can learn the truncated model in $\varepsilon$ total variation by drawing roughly $\operatorname{VC}(\mathcal{S}) / \varepsilon$ samples. We will use the following standard fact whose proof may be found for example in page 398 of [SSBD14]. For convenience we restate the result using our notation.

Lemma 21 ([SSBD14]). Let $D$ be a distribution on $\mathbb{R}^{d}$. Let $\mathcal{S}$ be a family of subsets of $\mathbb{R}^{d}$. Fix $\varepsilon \in(0,1), \delta \in(0,1 / 4)$ and let $N=O(\operatorname{VC}(\mathcal{S}) \log (1 / \varepsilon) / \varepsilon+\log (1 / \delta))$ Then, with probability at least $1-\delta$ over a choice of a sample $X \sim D^{N}$ we have that if $D(S) \geq \varepsilon$ then $|S \cap X| \neq \varnothing$.

\section{Proof of Lemma 1}

We define the class of sets $\mathcal{A}=\left\{S^{*} \backslash S: S \in \mathcal{S}\right\}$. We first argue that for any $A \subset \mathbb{R}^{d}$ we have $\mathrm{VC}(\mathcal{A}) \leq \mathrm{VC}(\mathcal{S})$. Let $X \subset \mathbb{R}^{d}$ be a set of points. The set of different labellings of $X$ using sets of $\mathcal{S}$ resp. $\mathcal{A}$ is $L_{\mathcal{S}}=\{X \cap S: S \in \mathcal{S}\}$ resp. $L_{\mathcal{A}}=\{X \cap S: S \in \mathcal{A}\}=\{X \cap(A \backslash S): S \in \mathcal{S}\}$. We define the function $g: L_{\mathcal{A}} \rightarrow L_{\mathcal{S}}$ by $g(X \cap(A \backslash S))=X \cap S$. We that observe for $S_{1}, S_{2} \in \mathcal{S}$ we have that $X \cap S_{1}=X \cap S_{2}$ implies that $X \cap\left(A \backslash S_{1}\right)=X \cap\left(A \backslash S_{2}\right)$. Therefore, $g$ is one-to-one and we obtain that $\left|L_{\mathcal{A}}\right| \leq\left|L_{\mathcal{S}}\right|$. We draw $N$ samples $X=\left\{x_{i}, i \in N\right\}$. Applying Lemma 21 for the family $\mathcal{A}$, we have that with $N$ samples, with probability at least $1-\delta$ it holds that if $\mathcal{N}\left(\boldsymbol{\mu}, \Sigma ; S^{*} \backslash S\right) \geq \varepsilon$ for some set $S \in \mathcal{S}$ then $\left|\left(S^{*} \backslash S\right) \cap X\right|>0$. Therefore, every set that is consistent with the samples, i.e. every $S$ that that contains the samples, satisfies the property $\mathcal{N}\left(\mu, \Sigma ; S^{*} \backslash S\right) \leq \varepsilon$. Moreover, since $d_{\mathrm{TV}}(\mathcal{N}(\widetilde{\boldsymbol{\mu}}, \widetilde{\Sigma}), \mathcal{N}(\boldsymbol{\mu}, \boldsymbol{\Sigma})) \leq \varepsilon$ we obtain that $\mathcal{N}\left(\widetilde{\mu}, \widetilde{\Sigma}, S^{*} \backslash S\right) \leq 2 \varepsilon$ for any set $S$ consistent with the data.

Next, we use the fact that $\widetilde{S}$ is chosen so that $\mathcal{N}\left(\widetilde{\mu}, \widetilde{\Sigma}, S^{*}\right) \geq \mathcal{N}(\widetilde{\mu}, \widetilde{\Sigma}, \widetilde{S})$. This means that for all $x \in S^{*} \cap \widetilde{S}$ it holds $\mathcal{N}\left(\widetilde{\mu}, \widetilde{\Sigma}, S^{*} ; x\right) \leq \mathcal{N}(\widetilde{\mu}, \widetilde{\Sigma}, \widetilde{S} ; x)$. To simplify notation we set $\widetilde{\mathcal{N}}_{\widetilde{S}}=\mathcal{N}(\widetilde{\mu}, \widetilde{\Sigma}, \widetilde{S})$, $\widetilde{\mathcal{N}}_{S^{*}}=\mathcal{N}\left(\widetilde{\mu}, \widetilde{\Sigma}, S^{*}\right)$, and $\mathcal{N}_{S^{*}}=\mathcal{N}\left(\mu, \Sigma, S^{*}\right)$. We have

$$
2 d_{\mathrm{TV}}\left(\widetilde{\mathcal{N}}_{\widetilde{S}^{\prime}} \widetilde{\mathcal{N}}_{S^{*}}\right)=\int_{\widetilde{\mathcal{N}}_{S^{*}}(x) \geq \widetilde{\mathcal{N}}_{\widetilde{S}(x)}}\left(\widetilde{\mathcal{N}}_{S^{*}}(\boldsymbol{x})-\widetilde{\mathcal{N}}_{\widetilde{S}}(\boldsymbol{x})\right) \mathrm{d} x \leq \int_{S^{*} \backslash \widetilde{S}} \widetilde{\mathcal{N}}_{S^{*}}(\boldsymbol{x}) \mathrm{d} x \leq \frac{\mathcal{N}\left(\widetilde{\boldsymbol{\mu}}, \widetilde{\Sigma} ; S^{*} \backslash \widetilde{S}\right)}{\alpha} \leq \frac{\varepsilon}{\alpha} .
$$

Moreover,

$$
d_{\mathrm{TV}}\left(\widetilde{\mathcal{N}}_{S^{*}}, \mathcal{N}_{S^{*}}\right) \leq \frac{d_{\mathrm{TV}}(\mathcal{N}(\widetilde{\boldsymbol{\mu}}, \widetilde{\boldsymbol{\Sigma}}), \mathcal{N}(\boldsymbol{\mu}, \boldsymbol{\Sigma}))}{\alpha} \leq \frac{\varepsilon}{\alpha}
$$

Using the triangle inequality we obtain that $d_{\mathrm{TV}}\left(\mathcal{N}(\widetilde{\mu}, \widetilde{\Sigma}, \widetilde{S}), \mathcal{N}\left(\mu, \Sigma, S^{*}\right)\right) \leq 3 \varepsilon /(2 \alpha)$.

\section{Proof of Lemma 2}

Using Lemma 18 we know that we can draw $\widetilde{O}\left(d^{2} \log ^{2}(1 / \alpha \delta)\right)$ samples and obtain estimates of the conditional mean and covariance $\widetilde{\mu}_{C}, \widetilde{\Sigma}_{C}$. Transforming the space so that $\widetilde{\mu}_{C}=0$ and $\widetilde{\Sigma}_{C}=\boldsymbol{I}$. For simplicity we will keep denoting the parameters of the unknown Gaussian $\mu, \Sigma$ after transforming the space. From Lemma 18 we have that $\left\|\Sigma^{-1 / 2} \mu\right\|_{2} \leq O\left(\log (1 / \alpha)^{1 / 2} / \alpha\right), \Omega\left(\alpha^{2}\right) \leq$ $\left\|\Sigma^{-1 / 2}\right\|_{2} \leq O\left(1 / \alpha^{2}\right)$ and $\|I-\Sigma\|_{F} \leq O\left(\log (1 / \alpha) / \alpha^{2}\right)$. Therefore, the cube of $\mathbb{R}^{d+d^{2}}$ where all the parameters $\mu_{i}, \Sigma_{i j}$ of the mean and the covariance lie has side length at most $O(1 / \operatorname{poly}(a))$. We can partition this cube into smaller cubes of side length $O(\varepsilon$ poly $(a) / d)$ and obtain that 
there exists a point of the grid $(\boldsymbol{u}, \boldsymbol{B})$ such that $\left\|\Sigma^{-1 / 2}(\boldsymbol{u}-\boldsymbol{\mu})\right\|_{2} \leq \varepsilon,\left\|\boldsymbol{I}-\boldsymbol{\Sigma}^{-1 / 2} \boldsymbol{B} \boldsymbol{\Sigma}^{-1 / 2}\right\|_{F} \leq \varepsilon$, which implies that $d_{\mathrm{TV}}(\mathcal{N}(\boldsymbol{u}, \boldsymbol{B}), \mathcal{N}(\boldsymbol{\mu}, \boldsymbol{\Sigma})) \leq \varepsilon$. Assume now that for each guess $(\boldsymbol{u}, \boldsymbol{B})$ of our grid we solve the optimization problem as defined in Lemma 1 and find a candidate set $S_{u, B}$. Notice that the set of our hypotheses $u, B, S_{u, B}$ is $O\left(\left(d^{2} / \varepsilon\right)^{d^{2}+d}\right)$. Moreover, using Lemma 1 and the fact that there exists a point $\boldsymbol{u}, \boldsymbol{B})$ in the grid so that $d_{\mathrm{TV}}(\mathcal{N}(\boldsymbol{u}, \boldsymbol{B}), \mathcal{N}(\boldsymbol{\mu}, \boldsymbol{\Sigma})) \leq \varepsilon$, we obtain that $d_{\mathrm{TV}}\left(\mathcal{N}\left(\boldsymbol{u}, \boldsymbol{B}, S_{\boldsymbol{u}, \boldsymbol{B}}\right), \mathcal{N}(\boldsymbol{\mu}, \boldsymbol{\Sigma}, S)\right) \leq \varepsilon$. Now we can use Lemma 20 we can select a hypotheses $\mathcal{N}(\boldsymbol{u}, \boldsymbol{B}, \widetilde{S})$ within $O(\varepsilon)$ total variation distance of $\mathcal{N}(\boldsymbol{\mu}, \boldsymbol{\Sigma}, S)$, and the number samples required to run the tournament is as claimed.

\section{C $\quad$ Missing Proofs of Section 4.1}

To prove Theorem 3 we shall use the inequalities of Lemma 22.

Lemma 22. Let $k \in \mathbb{N}$. Then for all $0<x<\frac{2 k+1}{2 k}$ it holds,

$$
-k \log x-\frac{1}{2} \log \left(1-2 k(x-1) \leq 2 k^{2}(x-1)^{2}\left(\frac{1}{x}+\frac{1}{1-2 k(x-1)}\right)\right.
$$

Moreover, for all $x>\frac{2 k-1}{2 k}$

$$
k \log x-\frac{1}{2} \log (1-2 k(1-x)) \leq k^{2}(1-x)^{2}\left(1+\frac{1}{1-2 k(1-x)}\right) .
$$

Proof. We start with the first inequality. Let $f(x)=-k \log x-\frac{1}{2} \log (1-2 k(x-1)$. We first assume that $1 \leq x \frac{2 k+1}{2 k}$. We have

$$
\begin{aligned}
f(x) & =\int_{1}^{x}\left(\frac{k}{1-2 k(t-1)}-\frac{k}{t}\right) \mathrm{d} t \\
& =k(1+2 k) \int_{1}^{x} \frac{t-1}{t(1-2 k(t-1))} \mathrm{d} t \\
& \leq \frac{k(1+2 k)}{1-2 k(x-1)} \int_{1}^{x}(t-1) \mathrm{d} t \\
& \leq 2 k^{2} \frac{(x-1)^{2}}{1-2 k(x-1)}
\end{aligned}
$$

If $0<x \leq 1$ we have

$$
f(x) \leq \frac{k(1+2 k)}{x} \int_{1}^{x}(t-1) \mathrm{d} t \leq 2 k^{2} \frac{(x-1)^{2}}{x}
$$

Adding these two bounds gives an upper bound for all $0<x<\frac{2 k+1}{2 k}$. Similarly, we now show the second inequality. Let $g(x)=k \log x-\frac{1}{2} \log (1-2 k(1-x))$. We first assume that $1 \leq x$ and write

$$
\begin{aligned}
g(x) & =\int_{1}^{x}\left(\frac{k}{t}-\frac{k}{1-2 k(1-t)}\right) \mathrm{d} t \\
& =k \int_{1}^{x} \frac{(t-1)(2 k-1)}{t(1+2 k(t-1))} \mathrm{d} t \\
& \leq k(2 k-1) \int_{1}^{x} \frac{t-1}{\mathrm{~d}} t \\
& \leq k^{2}(x-1)^{2} .
\end{aligned}
$$


Similarly, if $\frac{2 k-1}{2 k}<x \leq 1$ we have

$$
g(x) \leq k^{2} \frac{(1-x)^{2}}{1-2 k(1-x)}
$$

We add the two bounds together to get the desired upper bound.

\section{Proof of Lemma 4}

For simplicity we denote $\mathcal{N}_{i}=\mathcal{N}\left(\boldsymbol{\mu}_{1}, \boldsymbol{\Sigma}_{i}\right)$. We start by proving the upper bound. Using Schwarz's inequality we write

$$
\underset{x \sim \mathcal{N}_{0}}{\mathbb{E}}\left[\left(\frac{\mathcal{N}_{1}(\boldsymbol{x})}{\mathcal{N}_{0}(\boldsymbol{x})}\right)^{k}\left(\frac{\mathcal{N}_{0}(\boldsymbol{x})}{\mathcal{N}_{2}(\boldsymbol{x})}\right)^{k}\right] \leq\left(\underset{x \sim \mathcal{N}_{0}}{\mathbb{E}}\left(\frac{\mathcal{N}_{1}(\boldsymbol{x})}{\mathcal{N}_{0}(\boldsymbol{x})}\right)^{2 k}\right)^{1 / 2}\left(\underset{x \sim \mathcal{N}_{0}}{\mathbb{E}}\left(\frac{\mathcal{N}_{0}(\boldsymbol{x})}{\mathcal{N}_{2}(\boldsymbol{x})}\right)^{2 k}\right)^{1 / 2} .
$$

We can now bound each term independently. We start by the ratio of $\mathcal{N}_{1} / \mathcal{N}_{0}$. Without loss of generality we may assume that $\Sigma^{1}$ is diagonal, $\Sigma_{1}=\operatorname{diag}\left(\lambda_{1}, \ldots, \lambda_{d}\right)$. We also let $\mu_{1}=\left(\mu_{1}, \ldots, \mu_{d}\right)$. We write

$$
\begin{aligned}
\underset{x \sim \mathcal{N}_{0}}{\mathbb{E}}\left[\left(\frac{\mathcal{N}_{1}(\boldsymbol{x})}{\mathcal{N}_{0}(\boldsymbol{x})}\right)^{2 k}\right] & =\frac{1}{\left|\boldsymbol{\Sigma}_{1}\right|^{k}} \underset{\boldsymbol{x} \sim \mathcal{N}_{0}}{\mathbb{E}}\left[\exp \left(-k\left(\boldsymbol{x}-\boldsymbol{\mu}_{1}\right)^{T} \boldsymbol{\Sigma}_{1}{ }^{-1}\left(\boldsymbol{x}-\boldsymbol{\mu}_{1}\right)+k \boldsymbol{x}^{T} \boldsymbol{x}\right)\right] \\
& =\frac{\exp \left(-k \boldsymbol{\mu}_{1}{ }^{T} \boldsymbol{\Sigma}_{1}{ }^{-1} \boldsymbol{\mu}_{1}\right)}{\left|\boldsymbol{\Sigma}_{1}\right|^{k}} \underset{x \sim \mathcal{N}_{0}}{\mathbb{E}}\left[\exp \left(k \boldsymbol{x}^{T}\left(\boldsymbol{I}-\boldsymbol{\Sigma}_{1}^{-1}\right) \boldsymbol{x}+2 k \boldsymbol{\mu}_{1}^{T} \boldsymbol{\Sigma}_{1}{ }^{-1} \boldsymbol{x}\right)\right] \\
& \leq \frac{1}{\left|\boldsymbol{\Sigma}_{1}\right|^{k}} \underset{x \sim \mathcal{N}_{0}}{\mathbb{E}}\left[\exp \left(\sum_{i=1}^{d}\left(k\left(1-1 / \lambda_{i}\right) x_{i}^{2}+2 k \frac{\mu_{i}}{\lambda_{i}} x_{i}\right)\right)\right] \\
& =\underbrace{\prod_{i=1}^{d} \frac{1}{\lambda_{i}^{k}} \underset{x \sim \mathcal{N}_{0}}{\mathbb{E}}\left[\exp \left(k\left(1-1 / \lambda_{i}\right) x^{2}+2 k \frac{\mu_{i}}{\lambda_{i}} x\right)\right]}_{A}
\end{aligned}
$$

We now use the fact that for all $a<1 / 2$.

$$
\underset{x \sim \mathcal{N}_{0}}{\mathbb{E}}\left[\exp \left(a x^{2}+b x\right)\right]=\frac{1}{\sqrt{1-2 a}} \exp \left(\frac{b^{2}}{2-4 a}\right)
$$

At this point notice that since for all $i$ it holds $\lambda_{i}<2 k /(2 k-1)$ we have that term $A$ is bounded. We get that

$$
A=\underbrace{\exp \left(\sum_{i=1}^{d}\left(k \log \frac{1}{\lambda_{i}}-\frac{1}{2} \log \left(1-2 k\left(1-\frac{1}{\lambda_{i}}\right)\right)\right)\right)}_{A_{1}} \underbrace{\exp \left(\sum_{i=1}^{d} \frac{2 k^{2} \mu_{i}^{2}}{\lambda_{i}^{2}\left(1-2 k\left(1-1 / \lambda_{i}\right)\right)}\right)}_{A_{2}}
$$

To bound the term $A_{1}$ we use the second inequality of Lemma 22 to get

$$
A_{1} \leq \exp \left(\sum_{i=1}^{d} k^{2}\left(1-1 / \lambda_{i}\right)^{2}\left(1+\frac{1}{1-2 k\left(1-1 / \lambda_{i}\right)}\right)\right) \leq \exp \left(\frac{2 k^{2} B}{\delta}\right)
$$


Bounding $A_{2}$ is easier

$$
A_{2} \leq \exp \left(\frac{2 k^{2}\left\|\mu_{1}\right\|_{2}^{2}}{\lambda_{\min }^{2} \delta}\right)
$$

Combining the bounds for $A_{1}$ and $A_{2}$ we obtain

$$
\underset{x \sim \mathcal{N}_{0}}{\mathbb{E}}\left[\left(\frac{\mathcal{N}_{1}(\boldsymbol{x})}{\mathcal{N}_{0}(\boldsymbol{x})}\right)^{2 k}\right] \leq \exp \left(\frac{10 k^{2}}{\delta} B\right)
$$

We now work similarly to bound the ratio $\mathcal{N}_{0} / \mathcal{N}_{2}$. We will again assume that $\Sigma_{2}=\operatorname{diag}\left(\lambda_{1}, \ldots, \lambda_{d}\right)$ and $\mu_{2}=\left(\mu_{1}, \ldots, \mu_{d}\right)$. We have

$$
\begin{aligned}
\underset{x \sim \mathcal{N}_{0}}{\mathbb{E}}\left[\left(\frac{\mathcal{N}_{0}(x)}{\mathcal{N}_{2}(x)}\right)^{2 k}\right] & =\exp \left(k \boldsymbol{\mu}_{2}^{T} \Sigma_{2}^{-1} \boldsymbol{\mu}_{2}\right) \underset{x \sim \mathcal{N}_{0}}{\mathbb{E}}\left[\left|\boldsymbol{\Sigma}_{2}\right|^{k} \exp \left(k \boldsymbol{x}^{T}\left(\boldsymbol{\Sigma}_{2}^{-1}-\boldsymbol{I}\right) \boldsymbol{x}-2 k \boldsymbol{\mu}_{2} \boldsymbol{\Sigma}_{2}^{-1} \boldsymbol{x}\right)\right] \\
& \leq \exp ((k+1) B) \prod_{i=1}^{d} \underset{x \sim \mathcal{N}_{0}}{\mathbb{E}}\left[\exp \left(k\left(1 / \lambda_{i}-1\right) x^{2}-k \log \left(1 / \lambda_{i}\right)-2 k\left(\mu_{i} / \lambda_{i}\right) x\right)\right] \\
& =\exp \left(\left(\frac{8 k^{2}}{\delta}+k+1\right) B\right) \exp \left(\sum_{i=1}^{d}\left(-k \log \left(1 / \lambda_{i}\right)-\frac{1}{2} \log \left(1-2 k\left(1 / \lambda_{i}-1\right)\right)\right)\right) \\
& \leq \exp \left(\left(\frac{10 k^{2}}{\delta}+4 k^{2}+k+1\right) B\right),
\end{aligned}
$$

where to obtain the last inequality we used the first inequality of Lemma 22 and the bounds for the maximum and minimum eigenvalues of $\Sigma_{2}$. Finally, plugging in the bounds for the two ratios we get for $i=1,2$

$$
\underset{\boldsymbol{x} \sim \mathcal{N}_{0}}{\mathbb{E}}\left[\left(\frac{\mathcal{N}_{3-i}(\boldsymbol{x})}{\mathcal{N}_{i}(\boldsymbol{x})}\right)^{k}\right] \leq \exp \left(\frac{13 k^{2}}{\delta} B\right) .
$$

Having the upper bound it is now easy to prove the lower bound using the convexity of $x \mapsto x^{-1}$ and Jensen's inequality.

$$
\underset{x \sim \mathcal{N}_{0}}{\mathbb{E}}\left[\left(\frac{\mathcal{N}_{1}(\boldsymbol{x})}{\mathcal{N}_{2}(\boldsymbol{x})}\right)^{k}\right]=\underset{x \sim \mathcal{N}_{0}}{\mathbb{E}}\left[\left(\frac{\mathcal{N}_{2}(\boldsymbol{x})}{\mathcal{N}_{1}(\boldsymbol{x})}\right)^{-k}\right] \geq\left(\underset{x \sim \mathcal{N}_{0}}{\mathbb{E}}\left[\left(\frac{\mathcal{N}_{2}(\boldsymbol{x})}{\mathcal{N}_{1}(\boldsymbol{x})}\right)^{k}\right]\right)^{-1} \geq \exp \left(-\frac{13 k^{2}}{\delta} B\right)
$$

\section{Proof of Lemma 5}

For any $\rho \in(0,1)$, using identity 15 , we write

$$
\underset{x \sim \mathcal{N}_{0}}{\mathbb{E}}\left[f(x) T_{1-\rho}(x)\right]=\sum_{V \in \mathbb{N}^{d}}(1-\rho)^{|V|} \widehat{f}(V)^{2}
$$




$$
\begin{aligned}
\underset{x \sim \mathcal{N}(0, I)}{\mathbb{E}}\left[f(\boldsymbol{x})^{2}-f(\boldsymbol{x}) T_{1-\rho} f(\boldsymbol{x})\right] & =\sum_{V \in \mathbb{N}^{d}} \widehat{f}(V)^{2}-\sum_{V \in \mathbb{N}^{d}}(1-\rho)^{|V|} \widehat{f}(V)^{2} \\
& =\sum_{V \in \mathbb{N}^{d}}\left(1-(1-\rho)^{|V|}\right) \widehat{f}(V)^{2} \\
& \geq \sum_{|V| \geq 1 / \rho}\left(1-(1-\rho)^{|V|}\right) \widehat{f}(V)^{2} \\
& \geq \sum_{|V| \geq 1 / \rho}\left(1-(1-\rho)^{1 / \rho}\right) \widehat{f}(V)^{2} \\
& \geq(1-1 / \mathrm{e}) \sum_{|V| \geq 1 / \rho} \widehat{f}(V)^{2}
\end{aligned}
$$

\section{Proof of lemma 7}

We first write

$$
\frac{1}{2} \underset{(x, z) \sim D_{\rho}}{\mathbb{E}}\left[(r(\boldsymbol{x})-r(\boldsymbol{z}))^{2}\right]=\frac{1}{2} \underset{(x, z) \sim D_{\rho}}{\mathbb{E}}\left[\frac{r(\boldsymbol{x})^{2}}{2}+\frac{r(\boldsymbol{z})^{2}}{2}-r(\boldsymbol{x}) r(\boldsymbol{z})\right]=\underset{(x, z) \sim D_{\rho}}{\mathbb{E}}\left[r(\boldsymbol{x})^{2}-r(\boldsymbol{z}) r(\boldsymbol{x})\right] .
$$

Let

$$
\sum_{V \in \mathbb{N}^{d}} \widehat{r}(V) H_{V}(\boldsymbol{x})
$$

be the Hermite expansion of $r(\boldsymbol{x})$. From Parseval's identity and the Hermite expansion of OrnsteinâĂŞUhlenbeck operator, (15) we have

$$
\begin{aligned}
\underset{(x, z) \sim D_{\rho}}{\mathbb{E}}\left[r(\boldsymbol{x})^{2}-r(\boldsymbol{x}) r(\boldsymbol{z})\right] & =\sum_{V \in \mathbb{N}^{d}} \widehat{r}(V)^{2}-\sum_{V \in \mathbb{N}^{d}}(1-\rho)^{|V|} \widehat{r}(V)^{2} \\
& \leq \rho \sum_{V \in \mathbb{N}^{d}}|V| \widehat{r}(V)^{2}
\end{aligned}
$$

where the last inequality follows from Bernoulli's inequality $1-\rho|V| \leq(1-\rho)^{|V|}$. We know that (see for example [Sze67])

$$
\frac{\partial}{\partial x_{i}} H_{V}(x)=\frac{\partial}{\partial x_{i}} \prod_{v_{i} \in V} H_{v_{i}}\left(x_{i}\right)=\prod_{v_{j} \in V \backslash v_{i}} H_{v_{j}}\left(x_{j}\right) \sqrt{v_{i}} H_{v_{i}-1}\left(x_{i}\right)
$$

Therefore,

$$
\frac{\partial r(\boldsymbol{x})}{\partial x_{i}}=\sum_{V \in \mathbb{N}^{d}} \widehat{r}(V) \sqrt{v_{i}} H_{v_{i}-1}\left(x_{i}\right) \prod_{v_{j} \in V \backslash v_{i}} H_{v_{j}}\left(x_{j}\right)
$$

From Parseval's identity we have

$$
\underset{x \sim \mathcal{N}(0, I)}{\mathbb{E}}\left[\left(\frac{\partial r(x)}{\partial x_{i}}\right)^{2}\right]=\sum_{V \in \mathbb{N}^{d}} \widehat{r}(V)^{2} v_{i}
$$

Therefore,

$$
\underset{x \sim \mathcal{N}(0, I)}{\mathbb{E}}\left[\|\nabla r(\boldsymbol{x})\|_{2}^{2}\right]=\sum_{V \in \mathbb{N}^{d}}|V| \widehat{r}(V)^{2}
$$

The lemma follows. 


\section{C.1 Learning the Hermite Expansion}

In this section we present a way to bound the variance of the empirical estimation of Hermite coefficients. To bound the variance of estimating Hermite polynomials we shall need a bound for the expected value of the fourth power of a Hermite polynomial.

Lemma 23. For any $V \in \mathbb{N}^{d}$ it holds $\mathbb{E}_{x \sim \mathcal{N}_{0}}\left[H_{V}^{4}(\boldsymbol{x})\right] \leq 9^{|V|}$.

Proof. We compute

$$
\begin{aligned}
\underset{x \sim \mathcal{N}_{0}}{\mathbb{E}}\left[H_{V}^{4}(x)\right] & =\prod_{v_{i} \in V} \underset{x \sim \mathcal{N}(0,1)}{\mathbb{E}}\left[H_{v_{i}}^{2}\left(x_{i}\right) H_{v_{i}}^{2}\left(x_{i}\right)\right] \\
& =\prod_{v_{i} \in V} \underset{x \sim \mathcal{N}(0,1)}{\mathbb{E}}\left[\left(\sum_{i=0}^{v_{i}}\left(\begin{array}{c}
v_{i} \\
r
\end{array}\right) \frac{\sqrt{2 r !}}{r !} H_{2 r}\left(x_{i}\right)\right)\left(\sum_{r=0}^{v_{i}}\left(\begin{array}{c}
v_{i} \\
r
\end{array}\right) \frac{\sqrt{2 r !}}{r !} H_{2 r}\left(x_{i}\right)\right)\right] \\
& =\prod_{v_{i} \in V} \sum_{r=0}^{v_{i}}\left(\begin{array}{c}
v_{i} \\
r
\end{array}\right)^{2} \frac{(2 r) !}{(r !)^{2}} \underset{x \sim \mathcal{N}(0,1)}{\mathbb{E}}\left[H_{2 r}\left(x_{i}\right)^{2}\right]=\prod_{v_{i} \in V} \sum_{r=0}^{v_{i}}\left(\begin{array}{c}
v_{i} \\
r
\end{array}\right)^{2} \frac{(2 r) !}{(r !)^{2}} \\
& \leq \prod_{v_{i} \in V} \sum_{r=0}^{v_{i}}\left(\begin{array}{c}
v_{i} \\
r
\end{array}\right)^{2} 2^{2 r} \leq \prod_{v_{i} \in V}\left(\sum_{r=0}^{v_{i}}\left(\begin{array}{c}
v_{i} \\
r
\end{array}\right) 2^{r}\right)^{2} \leq \prod_{v_{i} \in V} 9^{v_{i}}=9^{|V|} .
\end{aligned}
$$

In the above computation we used the formula for the product of two (normalized) Hermite polynomials

$$
H_{i}(x) H_{i}(x)=\sum_{r=0}^{v_{i}}\left(\begin{array}{c}
v_{i} \\
r
\end{array}\right) \frac{\sqrt{2 r !}}{r !} H_{2 r}\left(x_{i}\right),
$$

see, for example, [Sze67].

\section{Proof of Lemma 8}

We have

$$
\underset{x \sim \mathcal{N}_{S}^{*}}{\mathbb{E}}\left[\left(H_{V}(\boldsymbol{x})-c_{V}\right)^{2}\right]=\underset{x \sim \mathcal{N}_{S}^{*}}{\mathbb{E}}\left[H_{V}^{2}(\boldsymbol{x})\right]-c_{V}^{2} \leq \frac{1}{\alpha} \underset{x \sim \mathcal{N}^{*}}{\mathbb{E}_{V}}\left[H_{V}^{2}(\boldsymbol{x})\right]
$$

We have

$$
\begin{aligned}
\left|\underset{\boldsymbol{x} \sim \mathcal{N}^{*}}{\mathbb{E}}\left[H_{V}^{2}(\boldsymbol{x})\right]-1\right| & =\left|\underset{\boldsymbol{x} \sim \mathcal{N}^{*}}{\mathbb{E}}\left[H_{V}^{2}(\boldsymbol{x})\right]-\underset{x \sim \mathcal{N}_{0}}{\mathbb{E}}\left[H_{V}^{2}(\boldsymbol{x})\right]\right| \\
& \leq \int H_{V}^{2}(\boldsymbol{x})\left|\mathcal{N}^{*}(\boldsymbol{x})-\mathcal{N}_{0}(\boldsymbol{x})\right| \mathrm{d} \boldsymbol{x} \\
& =\int H_{V}^{2}(\boldsymbol{x}) \sqrt{\left.\mathcal{N}_{0}(\boldsymbol{x})\right)} \frac{\mathcal{N}^{*}(\boldsymbol{x})-\mathcal{N}_{0}(\boldsymbol{x}) \mid}{\sqrt{\mathcal{N}_{0}(\boldsymbol{x})} \mathrm{d} \boldsymbol{x}} \\
& \leq(\underbrace{\int H_{V}^{4}(\boldsymbol{x}) \mathcal{N}_{0}(\boldsymbol{x}) \mathrm{d} \boldsymbol{x}}_{A})^{1 / 2}(\underbrace{\int \frac{\left(\mathcal{N}^{*}(\boldsymbol{x})-\mathcal{N}_{0}(\boldsymbol{x})\right)^{2}}{\mathcal{N}_{0}(\boldsymbol{x})} \mathrm{d} \boldsymbol{x}}_{B})^{1 / 2}
\end{aligned}
$$


To bound term $A$ we use Lemma 23. Using Lemma 4 we obtain

$$
B \leq \underset{x \sim \mathcal{N}(\mathbf{0}, \boldsymbol{I})}{\mathbb{E}}\left[\left(\frac{\mathcal{N}^{*}(\boldsymbol{x})}{\mathcal{N}_{0}(\boldsymbol{x})}\right)^{2}\right] \leq \operatorname{poly}(1 / \alpha)
$$

The bound for the variance follows from the independence of the samples.

\section{Missing Proofs of Section 4.2}

\section{Proof of Lemma 11}

We have that $\left|M_{\psi_{k}}(\boldsymbol{u}, \boldsymbol{B})-M_{\psi}^{\prime}(\boldsymbol{u}, \boldsymbol{B})\right| \leq\left|M_{\psi_{k}}(\boldsymbol{u}, \boldsymbol{B})-M_{\psi}(\boldsymbol{u}, \boldsymbol{B})\right|+\left|M_{\psi}(\boldsymbol{u}, \boldsymbol{B})-M_{\psi}^{\prime}(\boldsymbol{u}, \boldsymbol{B})\right|$. For the first term we have that

$$
\begin{aligned}
\left|M_{\psi_{k}}(\boldsymbol{u}, \boldsymbol{B})-M_{\psi}^{\prime}(\boldsymbol{u}, \boldsymbol{B})\right| & \leq C_{\boldsymbol{u}, \boldsymbol{B}} \underset{\boldsymbol{x} \sim \mathcal{N}_{S}^{*}}{\mathbb{E}}\left[\frac{\mathcal{N}_{0}(\boldsymbol{x})}{\mathcal{N}_{\boldsymbol{u}, \boldsymbol{B}}(\boldsymbol{x})}\left|\psi_{k}(\boldsymbol{x})-\psi(\boldsymbol{x})\right|\right] \\
& \leq C_{\boldsymbol{u}, \boldsymbol{B}} \sqrt{\underset{\boldsymbol{x} \sim \mathcal{N}_{0}}{\mathbb{E}}\left[\left(\frac{\mathcal{N}_{S}^{*}(\boldsymbol{x})}{\mathcal{N}_{\boldsymbol{u}, \boldsymbol{B}}(\boldsymbol{x})}\right)^{2}\right] \cdot \underset{\boldsymbol{x} \sim \mathcal{N}_{0}}{\mathbb{E}}\left[\left(\psi_{k}(\boldsymbol{x})-\psi(\boldsymbol{x})\right)^{2}\right]} \\
& \leq \frac{C_{\boldsymbol{u}, \boldsymbol{B}}}{\alpha^{*}} \sqrt{\underset{\boldsymbol{x} \sim \mathcal{N}_{0}}{\mathbb{E}}\left[\left(\frac{\mathcal{N}^{*}(\boldsymbol{x})}{\mathcal{N}_{\boldsymbol{u}, \boldsymbol{B}}(\boldsymbol{x})}\right)^{2}\right] \cdot \underset{x \sim \mathcal{N}_{0}}{\mathbb{E}}\left[\left(\psi_{k}(\boldsymbol{x})-\psi(\boldsymbol{x})\right)^{2}\right]}
\end{aligned}
$$

now we can use Lemma 4, Lemma 9 and Theorem 4 to get

$$
\left|M_{\psi_{k}}(\boldsymbol{u}, \boldsymbol{B})-M_{\psi}^{\prime}(\boldsymbol{u}, \boldsymbol{B})\right| \leq \operatorname{poly}\left(1 / \alpha^{*}\right) \sqrt{\varepsilon}
$$

For the second term we have that

$$
\left|M_{\psi}(\boldsymbol{u}, \boldsymbol{B})-M_{\psi}^{\prime}(\boldsymbol{u}, \boldsymbol{B})\right| \leq\left|1-\frac{C_{u, B}^{\prime}}{C_{u, B}}\right| C_{u, \boldsymbol{B}} \underset{x \sim \mathcal{N}_{S}^{*}}{\mathbb{E}}\left[\frac{\mathcal{N}^{*}(\boldsymbol{x})}{\alpha^{*} \mathcal{N}_{u, B}(\boldsymbol{x})}\right]
$$

We need to bound

$$
\begin{aligned}
\left|1-\frac{C_{\boldsymbol{u}, \boldsymbol{B}}^{\prime}}{C_{\boldsymbol{u}, \boldsymbol{B}}}\right| & =\left|1-e^{\left.-\frac{1}{2}\left(\operatorname{tr}\left((\boldsymbol{B}-\boldsymbol{I})\left(\boldsymbol{\Sigma}_{S}+\boldsymbol{\mu}_{S} \boldsymbol{\mu}_{S}^{T}-\widetilde{\boldsymbol{\Sigma}}_{S}\right)\right)\right)-\boldsymbol{u}^{T} \boldsymbol{\mu}_{S}\right)}\right| \leq e^{\left.\mid \frac{1}{2}\left(\operatorname{tr}\left((\boldsymbol{B}-\boldsymbol{I})\left(\boldsymbol{\Sigma}_{S}+\boldsymbol{\mu}_{S} \boldsymbol{\mu}_{S}^{T}-\widetilde{\boldsymbol{\Sigma}}_{S}\right)\right)\right)-\boldsymbol{u}^{T} \boldsymbol{\mu}_{S}\right) \mid}-1 \\
& \leq e^{\frac{1}{2}\left(\|\boldsymbol{B}-\boldsymbol{I}\|\left\|_{F}\right\| \boldsymbol{\Sigma}_{S}+\boldsymbol{\mu}_{S} \boldsymbol{\mu}_{S}^{T}-\widetilde{\boldsymbol{\Sigma}}_{S}\left\|_{F}+\right\| \boldsymbol{u}\left\|_{2}\right\| \boldsymbol{\mu}_{S} \|_{2}\right)}-1 \leq\|\boldsymbol{B}-\boldsymbol{I}\|\left\|_{F}\right\| \boldsymbol{\Sigma}_{S}+\boldsymbol{\mu}_{S} \boldsymbol{\mu}_{S}^{T}-\widetilde{\boldsymbol{\Sigma}}_{S}\left\|_{F}+\right\| \boldsymbol{u}\left\|_{2}\right\| \boldsymbol{\mu}_{S} \|_{2}
\end{aligned}
$$

where the last inequality holds when $\|\boldsymbol{B}-\boldsymbol{I}\|_{F}\left\|\boldsymbol{\Sigma}_{S}+\boldsymbol{\mu}_{S} \boldsymbol{\mu}_{S}^{T}-\widetilde{\boldsymbol{\Sigma}}_{S}\right\|_{F}+\|\boldsymbol{u}\|_{2}\left\|\boldsymbol{\mu}_{S}\right\|_{2} \leq 1$. But we know that $(\boldsymbol{u}, \boldsymbol{B}) \in \mathcal{D}$ and hence $\|\boldsymbol{B}-\boldsymbol{I}\|_{F} \leq \operatorname{poly}\left(1 / \alpha^{*}\right),\|\boldsymbol{u}\|_{2} \leq \operatorname{poly}\left(1 / \alpha^{*}\right)$. Also from Section 2.1 we have that $\left\|\boldsymbol{\Sigma}_{S}+\boldsymbol{\mu}_{S} \boldsymbol{\mu}_{S}^{T}-\widetilde{\boldsymbol{\Sigma}}_{S}\right\|_{F} \leq \varepsilon$ and $\left\|\boldsymbol{\mu}_{S}\right\|_{2} \leq \varepsilon$ and we can set $\varepsilon$ to be any inverse polynomial in $1 / \alpha^{*}$ times $\varepsilon$. Hence we get

$$
\left|1-\frac{C_{u, B}^{\prime}}{C_{u, B}}\right| \leq \varepsilon
$$


Now we can also use Lemma 9 and Lemma 4 which imply that

$$
C_{u, B} \underset{x \sim \mathcal{N}_{S}^{*}}{\mathbb{E}}\left[\frac{\mathcal{N}^{*}(x)}{\alpha^{*} \mathcal{N}_{u, B}(x)}\right] \leq \operatorname{poly}\left(1 / \alpha^{*}\right)
$$

and therefore we have

$$
\left|M_{\psi}(\boldsymbol{u}, \boldsymbol{B})-M_{\psi}^{\prime}(\boldsymbol{u}, \boldsymbol{B})\right| \leq \operatorname{poly}\left(1 / \alpha^{*}\right) \varepsilon .
$$

Hence we can once again divide $\varepsilon$ by any polynomial of $1 / \alpha^{*}$ without increasing the complexity presented in Section 2.1 and the lemma follows.

\section{Proof of Lemma 11}

We apply successive Cauchy-Schwarz inequalities to separate the terms that appear in the expression for the squared norm of the gradient. We have that

$$
\begin{aligned}
& \underset{x \sim \mathcal{N}_{S}^{*}}{\mathbb{E}}\left[\|\boldsymbol{v}(\boldsymbol{u}, \boldsymbol{B})\|_{2}^{2}\right]=C_{\boldsymbol{u}, \boldsymbol{B}}^{2} \underset{x \sim \mathcal{N}_{S}^{*}}{\mathbb{E}}\left[\left(\left\|x x^{T}-\tilde{\boldsymbol{\Sigma}}_{S}-\tilde{\boldsymbol{\mu}}_{S} \tilde{\boldsymbol{\mu}}_{S}^{T}\right\|_{F}^{2}+\left\|\tilde{\boldsymbol{\mu}}_{S}-\boldsymbol{x}\right\|_{2}^{2}\right) \frac{\mathcal{N}_{0}^{2}(\boldsymbol{x})}{\mathcal{N}_{\boldsymbol{u}, \boldsymbol{B}}^{2}(x)} \psi_{k}^{2}(\boldsymbol{x})\right] \\
& =C_{u, B}^{2} \underset{x \sim \mathcal{N}_{0}}{\mathbb{E}}\left[\left(\left\|x x^{T}-\tilde{\boldsymbol{\Sigma}}_{S}-\tilde{\boldsymbol{\mu}}_{S} \tilde{\boldsymbol{\mu}}_{S}^{T}\right\|_{F}^{2}+\left\|\tilde{\boldsymbol{\mu}}_{S}-\boldsymbol{x}\right\|_{2}^{2}\right) \frac{\mathcal{N}_{0}(\boldsymbol{x}) \mathcal{N}_{S}^{*}(\boldsymbol{x})}{\mathcal{N}_{\boldsymbol{u}, \boldsymbol{B}}^{2}(x)} \psi_{k}^{2}(\boldsymbol{x})\right] \\
& \leq C_{u, B}^{2} \underset{x \sim \mathcal{N}_{0}}{\mathbb{E}}\left[\left(\left\|x x^{T}-\tilde{\Sigma}_{S}-\tilde{\mu}_{S} \tilde{\mu}_{S}^{T}\right\|_{F}^{2}+\left\|\tilde{\mu}_{S}-x\right\|_{2}^{2}\right) \frac{\mathcal{N}_{0}(x) \mathcal{N}_{S}^{*}(x)}{\mathcal{N}_{u, B}^{2}(x)}\right]^{1 / 2} \underset{x \sim \mathcal{N}_{0}}{\mathbb{E}}\left[\psi_{k}^{4}(x)\right]^{1 / 2} \\
& \leq C_{u, B}^{2} \underset{x \sim \mathcal{N}_{0}}{\mathbb{E}}\left[\left(\left\|x x^{T}-\tilde{\Sigma}_{S}-\tilde{\mu}_{S} \tilde{\mu}_{S}^{T}\right\|_{F}^{2}+\left\|\tilde{\mu}_{S}-x\right\|_{2}^{2}\right)^{2}\right]^{1 / 4} \\
& \underset{x \sim \mathcal{N}_{0}}{\mathbb{E}}\left[\frac{\mathcal{N}_{0}^{2}(\boldsymbol{x}) \mathcal{N}^{* 2}(\boldsymbol{x})}{\mathcal{N}_{u, B}^{4}(x)}\right]^{1 / 4} \underset{x \sim \mathcal{N}_{0}}{\mathbb{E}}\left[\psi_{k}^{4}(\boldsymbol{x})\right]^{1 / 2} \\
& \leq C_{u, B}^{2} \underset{x \sim \mathcal{N}_{0}}{\mathbb{E}}\left[\left(\left\|x x^{T}-\tilde{\boldsymbol{\Sigma}}_{S}-\tilde{\boldsymbol{\mu}}_{S} \tilde{\boldsymbol{\mu}}_{S}^{T}\right\|_{F}+\left\|\tilde{\boldsymbol{\mu}}_{S}-\boldsymbol{x}\right\|_{2}\right)^{4}\right]^{1 / 4} \\
& \underset{x \sim \mathcal{N}_{0}}{\mathbb{E}}\left[\frac{\mathcal{N}_{0}^{4}(\boldsymbol{x})}{\mathcal{N}_{u, B}^{4}(x)}\right]^{1 / 8} \underset{x \sim \mathcal{N}_{0}}{\mathbb{E}}\left[\frac{\mathcal{N}_{S}^{* 4}(\boldsymbol{x})}{\mathcal{N}_{u, B}^{4}(x)}\right]^{1 / 8} \underset{x \sim \mathcal{N}_{0}}{\mathbb{E}}\left[\psi_{k}^{4}(\boldsymbol{x})\right]^{1 / 2}
\end{aligned}
$$

We now bound each term separately.

- By Lemma 9, $C_{u, B}^{2} \leq \operatorname{poly}(1 / \alpha)$.

- Given that $\left(\tilde{\mu}_{S}, \tilde{\Sigma}_{S}\right)$ are near-isotropic,

$$
\begin{aligned}
\underset{x \sim \mathcal{N}_{0}}{\mathbb{E}}\left[\left(\left\|x x^{T}-\tilde{\boldsymbol{\Sigma}}_{S}-\tilde{\boldsymbol{\mu}}_{S} \tilde{\boldsymbol{\mu}}_{S}^{T}\right\|_{F}+\left\|\tilde{\boldsymbol{\mu}}_{S}-\boldsymbol{x}\right\|_{2}\right)^{4}\right]^{1 / 4} \\
\leq \underset{x \sim \mathcal{N}_{0}}{\mathbb{E}}\left[\left(\left\|x x^{T}\right\|_{F}+\left\|\tilde{\boldsymbol{\Sigma}}_{S}\right\|_{F}+\left\|\tilde{\boldsymbol{\mu}}_{S} \tilde{\boldsymbol{\mu}}_{S}^{T}\right\|_{F}+\left\|\tilde{\boldsymbol{\mu}}_{S}\right\|+\|x\|_{2}\right)^{4}\right]^{1 / 4} \leq d \operatorname{poly}(1 / \alpha) .
\end{aligned}
$$


- By Lemma 4,

$$
\underset{x \sim \mathcal{N}_{0}}{\mathbb{E}}\left[\frac{\mathcal{N}_{0}^{4}(\boldsymbol{x})}{\mathcal{N}_{u, \boldsymbol{B}}^{4}(x)}\right]^{1 / 8} \underset{x \sim \mathcal{N}_{0}}{\mathbb{E}}\left[\frac{\mathcal{N}_{S}^{* 4}(\boldsymbol{x})}{\mathcal{N}_{u, \boldsymbol{B}}^{4}(x)}\right]^{1 / 8} \leq \operatorname{poly}(1 / \alpha)
$$

- For the last term, we have

$$
\begin{aligned}
\underset{x \sim \mathcal{N}_{0}}{\mathbb{E}}\left[\psi_{k}^{4}(\boldsymbol{x})\right] & =\underset{x \sim \mathcal{N}_{0}}{\mathbb{E}}\left[\left(\sum_{0 \leq|V| \leq k} \tilde{c}_{V} H_{V}(\boldsymbol{x})\right)^{4}\right] \\
& \leq 2^{3} \sum_{0 \leq|V| \leq k} \tilde{c}_{V}^{4} \underset{x \sim \mathcal{N}_{0}}{\mathbb{E}}\left[H_{V}^{4}(\boldsymbol{x})\right] \\
& \leq 8\left(\sum_{0 \leq|V| \leq k} \tilde{c}_{V}^{2}\right)^{2} \cdot\left(\max _{0 \leq|V| \leq k}\left\{\underset{x \sim \mathcal{N}_{0}}{\mathbb{E}}\left[H_{V}^{4}(\boldsymbol{x})\right]\right\}\right)
\end{aligned}
$$

From Lemma 8 and the conditioning on the event that the estimators of the Hermite coefficients are accurate we have that $\left(\tilde{c}_{V}-c_{V}\right)^{2} \leq 1$ and hence we get the following.

$$
\underset{x \sim \mathcal{N}_{0}}{\mathbb{E}}\left[\psi_{k}^{4}(x)\right] \leq 2^{10} d^{2 k}\left(\sum_{0 \leq|V| \leq \infty} c_{V}^{2}\right)^{4} \cdot\left(\max _{0 \leq|V| \leq k}\left\{\underset{x \sim \mathcal{N}_{0}}{\mathbb{E}}\left[H_{V}^{4}(\boldsymbol{x})\right]\right\}\right)
$$

To bound $\mathbb{E}_{x \sim \mathcal{N}_{0}}\left[H_{V}^{4}(\boldsymbol{x})\right]$ we use Lemma 23. Moreover, from Parseval's identity we obtain that $\sum_{0 \leq|V| \leq \infty} c_{V}^{2}=\mathbb{E}_{x \sim \mathcal{N}_{0}} \psi^{2}(\boldsymbol{x})$. From Lemma 4 we get

$$
\underset{x \sim \mathcal{N}_{0}}{\mathbb{E}} \psi^{2}(\boldsymbol{x}) \leq \frac{1}{\alpha} \underset{x \sim N_{0}}{\mathbb{E}}\left(\frac{\mathcal{N}^{*}(\boldsymbol{x})}{\mathcal{N}_{0}(\boldsymbol{x})}\right)^{2}=\operatorname{poly}(1 / \alpha) .
$$

From Lemma 8 we obtain that $\max _{0 \leq|V| \leq k}\left\{\mathbb{E}_{\boldsymbol{x} \sim \mathcal{N}_{0}}\left[H_{V}^{4}(\boldsymbol{x})\right]\right\} \leq 2^{k}$. The result follows from the above estimates.

\section{Proof of Lemma 12}

We will prove this lemma in two steps, first we will prove

$$
\left|z^{T} \mathcal{H}_{M_{\psi_{k}}}(\boldsymbol{u}, \boldsymbol{B}) \boldsymbol{z}-\boldsymbol{z}^{T} \mathcal{H}_{M_{\psi}}(\boldsymbol{u}, \boldsymbol{B}) \boldsymbol{z}\right| \leq \lambda
$$

and then we will prove that

$$
z^{T} \mathcal{H}_{M_{\psi}}(\boldsymbol{u}, \boldsymbol{B}) z \geq 2 \lambda
$$

for some parameter $\lambda \geq \operatorname{poly}\left(\alpha^{*}\right)$. To prove (19) we define

$$
p(z ; x)=\left(z^{T}\left(\begin{array}{c}
\frac{1}{2}\left(x x^{T}-\tilde{\Sigma}_{S}-\tilde{\mu}_{S} \tilde{\mu}_{S}^{T}\right)^{b} \\
\tilde{\mu}_{S}-x
\end{array}\right)\right)^{2}
$$


and we have that

$$
\begin{aligned}
\left|\boldsymbol{z}^{T} \mathcal{H}_{M_{\psi_{k}}}(\boldsymbol{u}, \boldsymbol{B}) \boldsymbol{z}-\boldsymbol{z}^{T} \mathcal{H}_{M_{\psi}}(\boldsymbol{u}, \boldsymbol{B}) \boldsymbol{z}\right| \\
\quad=\underset{\boldsymbol{x} \sim \mathcal{N}_{S}^{*}}{\mathbb{E}}\left[\mathrm{e}^{h(\boldsymbol{u}, \boldsymbol{B} ; \boldsymbol{x})} \mathcal{N}(\mathbf{0}, \boldsymbol{I} ; \boldsymbol{x}) \cdot p(\boldsymbol{z} ; \boldsymbol{x}) \cdot\left|\psi_{k}(\boldsymbol{x})-\psi(\boldsymbol{x})\right|\right] \\
\quad=\underset{\boldsymbol{x} \sim \mathcal{N}_{0}}{\mathbb{E}}\left[\mathrm{e}^{h(\boldsymbol{u}, \boldsymbol{B} ; \boldsymbol{x})} \cdot \mathbf{1}_{S}(\boldsymbol{x}) \cdot \mathcal{N}^{*}(\boldsymbol{x}) \cdot p(\boldsymbol{z} ; \boldsymbol{x}) \cdot\left|\psi_{k}(\boldsymbol{x})-\psi(\boldsymbol{x})\right|\right]
\end{aligned}
$$

we then separate the terms using the Cauchy Schwarz inequality

$$
\begin{aligned}
& \left|\boldsymbol{z}^{T} \mathcal{H}_{M_{\psi_{k}}}(\boldsymbol{u}, \boldsymbol{B}) \boldsymbol{z}-\boldsymbol{z}^{T} \mathcal{H}_{M_{\psi}}(\boldsymbol{u}, \boldsymbol{B}) \boldsymbol{z}\right| \\
& \quad \leq \sqrt{\underset{\boldsymbol{x} \sim \mathcal{N}_{0}}{\mathbb{E}}\left[\mathrm{e}^{2 h(\boldsymbol{u}, \boldsymbol{B} ; \boldsymbol{x})} \cdot \mathbf{1}_{S}(\boldsymbol{x}) \cdot\left(\mathcal{N}^{*}(\boldsymbol{x})\right)^{2} \cdot p^{2}(\boldsymbol{z} ; \boldsymbol{x})\right]} \cdot \sqrt{\underset{\boldsymbol{x} \sim \mathcal{N}_{0}}{\mathbb{E}}\left[\left(\psi_{k}(\boldsymbol{x})-\psi(\boldsymbol{x})\right)^{2}\right]}
\end{aligned}
$$

we apply now the Hermite concentration from Theorem 4 and we get

$$
\begin{aligned}
& \leq \sqrt{\underset{x \sim \mathcal{N}_{0}}{\mathbb{E}}\left[\mathrm{e}^{2 h(u, B ; \boldsymbol{x})} \cdot \mathbf{1}_{S}(\boldsymbol{x}) \cdot\left(\mathcal{N}^{*}(\boldsymbol{x})\right)^{2} \cdot p^{2}(\boldsymbol{z} ; \boldsymbol{x})\right]} \cdot \sqrt{\varepsilon} \\
& \leq \sqrt[4]{\underset{x \sim \mathcal{N}^{*}}{\mathbb{E}}\left[\mathrm{e}^{4 h(u, B ; \boldsymbol{x})} \cdot \mathbf{1}_{S}(\boldsymbol{x}) \cdot\left(\mathcal{N}^{*}(\boldsymbol{x})\right)^{2}\left(\mathcal{N}_{0}(\boldsymbol{x})\right)^{2}\right]} \cdot \sqrt[4]{\underset{x \sim \mathcal{N}^{*}}{\mathbb{E}}\left[p^{4}(\boldsymbol{z} ; \boldsymbol{x})\right]} \cdot \sqrt{\varepsilon}
\end{aligned}
$$

we now use (7), Lemma 9 and the fact that $\mathbf{1}_{S}(\boldsymbol{x}) \leq 1$ to get

$$
\leq \sqrt[4]{\underset{x \sim \mathcal{N}^{*}}{\mathbb{E}}\left[\mathrm{e}^{4 h(u, B ; \boldsymbol{x})}\left(\mathcal{N}^{*}(\boldsymbol{x})\right)^{2}\left(\mathcal{N}_{0}(\boldsymbol{x})\right)^{2}\right]} \cdot \sqrt[4]{\underset{x \sim \mathcal{N}^{*}}{\mathbb{E}}\left[p^{4}(\boldsymbol{z} ; \boldsymbol{x})\right]} \cdot \sqrt{\varepsilon}
$$

and finally we use Lemma 4 to prove the following

$$
\left|\boldsymbol{z}^{T} \mathcal{H}_{M_{\psi_{k}}}(\boldsymbol{u}, \boldsymbol{B}) \boldsymbol{z}-\boldsymbol{z}^{T} \mathcal{H}_{M_{\psi}}(\boldsymbol{u}, \boldsymbol{B}) \boldsymbol{z}\right| \leq \sqrt[4]{\underset{\boldsymbol{x} \sim \mathcal{N}^{*}}{\mathbb{E}}\left[p^{4}(\boldsymbol{z} ; \boldsymbol{x})\right]} \cdot \operatorname{poly}\left(1 / \alpha^{*}\right) \cdot \sqrt{\varepsilon}
$$

Next we prove (20). We have that

$$
\begin{aligned}
\boldsymbol{z}^{T} \mathcal{H}_{M_{\psi}}(\boldsymbol{u}, \boldsymbol{B}) \boldsymbol{z} & =\underset{\boldsymbol{x} \sim \mathcal{N}_{S}^{*}}{\mathbb{E}}\left[\mathrm{e}^{h(\boldsymbol{u}, \boldsymbol{B} ; \boldsymbol{x})} \mathcal{N}(\mathbf{0}, \boldsymbol{I} ; \boldsymbol{x}) \cdot p(\boldsymbol{z} ; \boldsymbol{x}) \cdot \psi(\boldsymbol{x})\right] \\
& =\frac{1}{\alpha^{*}} C_{\boldsymbol{u}, \boldsymbol{B}} \underset{\boldsymbol{x} \sim \mathcal{N}_{S}^{*}}{\mathbb{E}}\left[\frac{\mathcal{N}^{*}(\boldsymbol{x})}{\mathcal{N}_{\boldsymbol{u}, \boldsymbol{B}}(\boldsymbol{x})} p(\boldsymbol{z} ; \boldsymbol{x})\right] .
\end{aligned}
$$

Now we define the set $\bar{Q}_{z}=\left\{\boldsymbol{x} \in \mathbb{R}^{d}|| p(\boldsymbol{z} ; \boldsymbol{x}) \mid \leq \frac{1}{32 \mathrm{C}}\left(\alpha^{*}\right)^{4} \sqrt[4]{\mathbb{E}_{\boldsymbol{x} \sim \mathcal{N}^{*}}\left[p^{4}(\boldsymbol{z} ; \boldsymbol{x})\right]}\right\}$, where $C$ is the universal constant guaranteed from Theorem 9. Then using Theorem 9 and the fact that $p(z ; x)$ has degree 4 we get that $\mathcal{N}\left(\boldsymbol{\mu}^{*}, \boldsymbol{\Sigma}^{*} ; \bar{Q}\right) \leq \frac{\alpha^{*}}{2}$. Hence we define the set $S^{\prime}=S \cap \bar{Q}$ and we have that $\mathcal{N}\left(\boldsymbol{\mu}^{*}, \boldsymbol{\Sigma}^{*} ; S^{\prime}\right) \geq \alpha^{*} / 2$.

$$
\begin{aligned}
z^{T} \mathcal{H}_{M_{\psi}}(\boldsymbol{u}, \boldsymbol{B}) z & \geq \frac{1}{\alpha^{*}} C_{\boldsymbol{u}, \boldsymbol{B}} \underset{x \sim \mathcal{N}_{S^{\prime}}^{*}}{\mathbb{E}}\left[\frac{\mathcal{N}^{*}(\boldsymbol{x})}{\mathcal{N}_{\boldsymbol{u}, \boldsymbol{B}}(\boldsymbol{x})} p(\boldsymbol{z} ; \boldsymbol{x})\right] \\
& \geq\left(\min _{\boldsymbol{x} \in S^{\prime}} p(\boldsymbol{z} ; \boldsymbol{x})\right) \frac{1}{\alpha^{*}} C_{\boldsymbol{u}, \boldsymbol{B}} \underset{x \sim \mathcal{N}_{S^{\prime}}^{*}}{\mathbb{E}}\left[\frac{\mathcal{N}^{*}(\boldsymbol{x})}{\mathcal{N}_{u, B}(\boldsymbol{x})}\right]
\end{aligned}
$$


and from the definition of $S^{\prime}$ and Lemma 9 we have that

$$
\boldsymbol{z}^{T} \mathcal{H}_{M_{\psi}}(\boldsymbol{u}, \boldsymbol{B}) z \geq \operatorname{poly}\left(\alpha^{*}\right) \cdot \underset{x \sim \mathcal{N}_{S^{\prime}}^{*}}{\mathbb{E}}\left[\frac{\mathcal{N}^{*}(\boldsymbol{x})}{\mathcal{N}_{\boldsymbol{u}, \boldsymbol{B}}(\boldsymbol{x})}\right] \cdot \sqrt[4]{\underset{x \sim \mathcal{N}^{*}}{\mathbb{E}}\left[p^{4}(\boldsymbol{z} ; \boldsymbol{x})\right]}
$$

now we can apply Jensen's inequality on the convex function $x \mapsto 1 / x$ and we get

$$
\begin{aligned}
\boldsymbol{z}^{T} \mathcal{H}_{M_{\psi}}(\boldsymbol{u}, \boldsymbol{B}) \boldsymbol{z} & \geq \operatorname{poly}\left(\alpha^{*}\right) \cdot \frac{1}{\mathbb{E}_{\boldsymbol{x} \sim \mathcal{N}_{S^{\prime}}^{*}\left[\frac{\mathcal{N}_{\boldsymbol{u}, \boldsymbol{B}}(\boldsymbol{x})}{\mathcal{N}^{*}(\boldsymbol{x})}\right]} \cdot \sqrt[4]{\underset{x \sim \mathcal{N}^{*}}{\mathbb{E}}\left[p^{4}(\boldsymbol{z} ; \boldsymbol{x})\right]}} \\
& \geq \operatorname{poly}\left(\alpha^{*}\right) \cdot \frac{1}{\sqrt{\mathbb{E}_{\boldsymbol{x} \sim \mathcal{N}^{*}}\left[\left(\frac{\mathcal{N}_{\boldsymbol{u}, \boldsymbol{B}}(\boldsymbol{x})}{\mathcal{N}^{*}(\boldsymbol{x})}\right)^{2}\right]}} \cdot \sqrt[4]{\underset{x \sim \mathcal{N}^{*}}{\mathbb{E}}\left[p^{4}(\boldsymbol{z} ; \boldsymbol{x})\right]}
\end{aligned}
$$

finally using Lemma 4 we get

$$
\boldsymbol{z}^{T} \mathcal{H}_{M_{\psi}}(\boldsymbol{u}, \boldsymbol{B}) \boldsymbol{z} \geq \operatorname{poly}\left(\alpha^{*}\right) \sqrt[4]{\underset{x \sim \mathcal{N}^{*}}{\mathbb{E}}\left[p^{4}(\boldsymbol{z} ; \boldsymbol{x})\right]}
$$

Now using (21) and (22) we can see that it is possible to pick $\varepsilon$ in the Hermite concentration to be the correct polynomial in $\alpha^{*}$ so that

$$
\left|z^{T} \mathcal{H}_{M_{\psi_{k}}}(\boldsymbol{u}, \boldsymbol{B}) z-\boldsymbol{z}^{T} \mathcal{H}_{M_{\psi}}(\boldsymbol{u}, \boldsymbol{B}) z\right| \leq \boldsymbol{z}^{T} \mathcal{H}_{M_{\psi}}(\boldsymbol{u}, \boldsymbol{B}) \boldsymbol{z}
$$

which implies from Jensen's inequality that

$$
\begin{aligned}
\boldsymbol{z}^{T} \mathcal{H}_{M_{\psi_{k}}}(\boldsymbol{u}, \boldsymbol{B}) \boldsymbol{z} & \geq \operatorname{poly}\left(\alpha^{*}\right) \sqrt[4]{\underset{x \sim \mathcal{N}^{*}}{\mathbb{E}}\left[p^{4}(\boldsymbol{z} ; \boldsymbol{x})\right]} \\
& \geq \operatorname{poly}\left(\alpha^{*}\right) \underset{\boldsymbol{x} \sim \mathcal{N}^{*}}{\mathbb{E}}[p(\boldsymbol{z} ; \boldsymbol{x})]
\end{aligned}
$$

So the last step is to prove a lower bound for $\mathbb{E}_{x \sim \mathcal{N}^{*}}[p(z ; x)]$. For this we can use the Lemma 3 of [DGTZ18] from which we can directly get $\mathbb{E}_{\boldsymbol{x} \sim \mathcal{N}^{*}}[p(\boldsymbol{z} ; \boldsymbol{x})] \geq \operatorname{poly}\left(\alpha^{*}\right)$ and the lemma follows.

\section{E Details of Section 4.3}

We present here the of the proof of Theorem 6. We already proved that given only positive examples from a truncated normal can obtain arbitrarily good estimations of the unconditional (true) parameters of the normal using Algorithm 1. Recall also that with positive samples we can obtain an approximation of the function $\psi(\boldsymbol{x})$ defined in 4 . From Theorem 4 we know that with $d^{\text {poly }(1 / \alpha) \Gamma(S)^{2} / \varepsilon^{4}}$ samples we can obtain a function $\psi_{k}(x)$ such that

$$
\underset{x \sim \mathcal{N}_{0}}{\mathbb{E}}\left[\left(\left(\psi_{k}(\boldsymbol{x})-\psi(\boldsymbol{x})\right)^{2}\right] \leq \varepsilon\right.
$$

Now we can construct an almost indicator function using $\psi_{k}$ and the learned parameters $\widetilde{\mu}, \widetilde{\boldsymbol{I}}$. We denote $\widetilde{\mathcal{N}}=\mathcal{N}(\widetilde{\mu}, \widetilde{\Sigma})$.

$$
\widetilde{f}(x)=\frac{\mathcal{N}_{0}(x)}{\widetilde{\mathcal{N}}(x)} \psi_{k}(x) .
$$


This function should be a good enough approximation to the function

$$
f(x)=\frac{\mathcal{N}_{0}(x)}{\mathcal{N}^{*}(x)} \psi(x)=\frac{\mathbf{1}_{S}(x)}{\alpha^{*}} .
$$

Notice that even though we do not know the mass of the truncation set $\alpha^{*}$ we can still construct a threshold function that achieves low error with respect to the zero-one loss. We first prove a standard lemma that upper bounds the zero-one loss with the distance of $f$ and $\widetilde{f}$. We prove it so that we have a version consistent with our notation.

Lemma 24. Let $S$ be a subset of $\mathbb{R}^{d}$. Let $D$ be a distribution on $\mathbb{R}^{d}$ and let $f: \mathbb{R}^{d} \rightarrow\{0, B\}$, where $B>1$ such that $f(\boldsymbol{x})=B \mathbf{1}_{S}(\boldsymbol{x})$. For any $g: \mathbb{R}^{d} \mapsto[0,+\infty)$ it holds $\left.\left.\mathbb{E}_{x \sim D}[\mathbf{1}\{g(x)>1 / 2)\} \neq \mathbf{1}_{S}(\boldsymbol{x})\right\}\right] \leq$ $\sqrt{2} \mathbb{E}_{x \sim D}[\sqrt{|g(x)-f(x)|}]$.

Proof. It suffices to show that for all $x \in \mathbb{R}^{d}$ it holds

$$
\mathbf{1}\{\operatorname{sgn}(g(x)-1 / 2) \neq S(x)\} \leq \sqrt{2} \sqrt{|g(x)-f(x)|} .
$$

We only need to consider the case where $\operatorname{sgn}(g(x)-1 / 2) \neq S(x)$. Assume first that $g(x)>1 / 2$ and $x \neq S$. Then the LHS of Equation (25) is 1 and the RHS of (25) is $\sqrt{2} \sqrt{|g(x)-f(x)|} \geq$ $\sqrt{2} \sqrt{|1 / 2-0|}=1$. Assume now that $g(x)<1 / 2$ and $S(x)=1$. Then the RHS of (25) equals $\sqrt{2} \sqrt{|g(x)-f(x)|} \geq \sqrt{2} \sqrt{|B-1 / 2|} \geq 1$.

We now state the following lemma that upper bounds the distance of $f$ and $\widetilde{f}$ in with the sum of the total variation distance of the true and learned distributions as well as the approximation error of $\psi_{k}$.

Lemma 25. Let $\alpha$ be the absolute constant of (3). Let $S \subseteq \mathbb{R}^{d}$ and let $\mathcal{N}^{*}, \widetilde{\mathcal{N}}$ be $(O(\log (1 / \alpha)), 1 / 16)$ isotropic. Let $\psi$ be as in (4). Moreover, let $\widetilde{f}, f$ be as in (23), (24). Then,

$$
\underset{x \sim \mathcal{N}^{*}}{\mathbb{E}}[\sqrt{|\widetilde{f}(x)-f(\boldsymbol{x})|}] \leq \operatorname{poly}(1 / \alpha)\left(\left(\underset{x \sim \mathcal{N}_{0}}{\mathbb{E}}\left[\left(\psi_{k}(\boldsymbol{x})-\psi(\boldsymbol{x})\right)^{2}\right]\right)^{1 / 4}+\left(d_{\mathrm{TV}}\left(\mathcal{N}^{*}, \widetilde{\mathcal{N}}\right)\right)^{1 / 4}\right)
$$

Proof. We compute

$$
\begin{aligned}
& \underset{x \sim \mathcal{N}^{*}}{\mathbb{E}}[\sqrt{|\widetilde{f}(\boldsymbol{x})-f(\boldsymbol{x})|}] \leq \underset{x \sim \mathcal{N}^{*}}{\mathbb{E}}\left[\left(\left|\psi_{k}(\boldsymbol{x}) \frac{\mathcal{N}_{0}(\boldsymbol{x})}{\widetilde{\mathcal{N}}(\boldsymbol{x})}-\psi(\boldsymbol{x}) \frac{\mathcal{N}_{0}(\boldsymbol{x})}{\mathcal{N}^{*}(\boldsymbol{x})}\right|\right)^{1 / 2}\right] \\
& =\underset{x \sim \mathcal{N}^{*}}{\mathbb{E}}\left[\left(\left|\psi_{k}(\boldsymbol{x}) \frac{\mathcal{N}_{0}(\boldsymbol{x})}{\widetilde{\mathcal{N}}(\boldsymbol{x})}-\psi(\boldsymbol{x}) \frac{\mathcal{N}_{0}(\boldsymbol{x})}{\widetilde{\mathcal{N}}(\boldsymbol{x})}+\psi(\boldsymbol{x}) \frac{\mathcal{N}_{0}(\boldsymbol{x})}{\widetilde{\mathcal{N}}(\boldsymbol{x})}-\psi(\boldsymbol{x}) \frac{\mathcal{N}_{0}(\boldsymbol{x})}{\mathcal{N}^{*}(\boldsymbol{x})}\right|\right)^{1 / 2}\right] \\
& \leq \underset{x \sim \mathcal{N}^{*}}{\mathbb{E}}\left[\left(\left|\psi_{k}(\boldsymbol{x})-\psi(\boldsymbol{x})\right| \frac{\mathcal{N}_{0}(\boldsymbol{x})}{\widetilde{\mathcal{N}}(\boldsymbol{x})}\right)^{1 / 2}\right]+\underset{x \sim \mathcal{N}^{*}}{\mathbb{E}}\left[\left(\psi(\boldsymbol{x})\left|\frac{\mathcal{N}_{0}(\boldsymbol{x})}{\widetilde{\mathcal{N}}(\boldsymbol{x})}-\frac{\mathcal{N}_{0}(\boldsymbol{x})}{\mathcal{N}^{*}(\boldsymbol{x})}\right|\right)^{1 / 2}\right]
\end{aligned}
$$

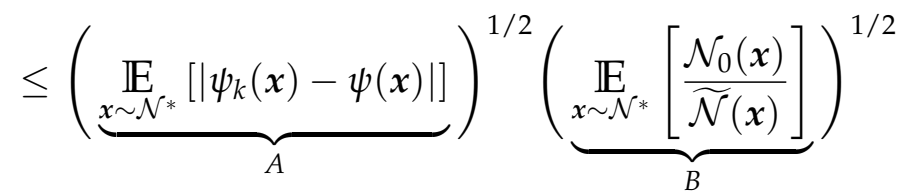

$$
\begin{aligned}
& +(\underbrace{\underset{x \sim \mathcal{N}^{*}}{\mathbb{E}}\left[\psi(x)\left|\frac{\mathcal{N}_{0}(x)}{\widetilde{\mathcal{N}}(x)}-\frac{\mathcal{N}_{0}(x)}{\mathcal{N}^{*}(\boldsymbol{x})}\right|\right]}_{C})^{1 / 2}
\end{aligned}
$$


where for term $C$ we used Jensen's inequality. Using Lemma 26 and Lemma 4 we have that

$A \leq\left(\underset{x \sim \mathcal{N}_{0}}{\mathbb{E}_{1}}\left[\left(\psi_{k}(\boldsymbol{x})-\psi(\boldsymbol{x})\right)^{2}\right]\right)^{1 / 2}\left(\underset{x \sim \mathcal{N}^{*}}{\mathbb{E}}\left[\frac{\mathcal{N}_{0}(\boldsymbol{x})}{\mathcal{N}^{*}(\boldsymbol{x})}\right]\right)^{1 / 2} \leq\left(\underset{x \sim \mathcal{N}_{0}}{\mathbb{E}}\left[\left(\psi_{k}(\boldsymbol{x})-\psi(\boldsymbol{x})\right)^{2}\right]\right)^{1 / 2} \operatorname{poly}(1 / \alpha)$

Since $\mathcal{N}_{0}, \widetilde{\mathcal{N}}$, and $\mathcal{N}^{*}$ are $(O(\log (1 / \alpha), 1 / 16)$-isotropic, using Lemma 4 we obtain that

$$
B=\underset{x \sim \mathcal{N}_{0}}{\mathbb{E}}\left[\frac{\mathcal{N}_{0}(x)}{\widetilde{\mathcal{N}}(x)} \frac{\mathcal{N}^{*}(x)}{\widetilde{\mathcal{N}}(x)}\right] \leq\left(\underset{x \sim \mathcal{N}_{0}}{\mathbb{E}}\left[\frac{\mathcal{N}_{0}(x)}{\widetilde{\mathcal{N}}(x)}\right]\right)^{1 / 2}\left(\underset{x \sim \mathcal{N}_{0}}{\mathbb{E}}\left[\frac{\mathcal{N}^{*}(x)}{\widetilde{\mathcal{N}}(x)}\right]\right)^{1 / 2} \leq \operatorname{poly}(1 / \alpha)
$$

We now bound term $C$. We write

$$
C=\underset{x \sim \mathcal{N}^{*}}{\mathbb{E}}\left[\psi(x)\left|\frac{\mathcal{N}_{0}(x)}{\widetilde{\mathcal{N}}(\boldsymbol{x})}-\frac{\mathcal{N}_{0}(\boldsymbol{x})}{\mathcal{N}^{*}(\boldsymbol{x})}\right|\right]=\frac{1}{\alpha^{*}} \underset{x \sim \mathcal{N}^{*}}{\mathbb{E}}\left[\left|\frac{\mathcal{N}^{*}(\boldsymbol{x})}{\widetilde{\mathcal{N}}(\boldsymbol{x})}-1\right|\right]
$$

To simplify notation, let $\ell(x)=\left|\frac{\mathcal{N}^{*}(x)}{\overline{\mathcal{N}}(x)}-1\right|$. Moreover, notice that $\mathbb{E}_{\boldsymbol{x} \sim \widetilde{\mathcal{N}}}[\ell(\boldsymbol{x})]=d_{\mathrm{TV}}\left(\mathcal{N}^{*}, \widetilde{\mathcal{N}}\right)$. Using the second bound of Lemma 26 and Lemma 4 we obtain

$$
C \leq \frac{1}{\alpha} d_{\mathrm{TV}}\left(\mathcal{N}^{*}, \widetilde{\mathcal{N}}\right)+\operatorname{poly}(1 / \alpha) \sqrt{d_{\mathrm{TV}}\left(\mathcal{N}^{*}, \widetilde{\mathcal{N}}\right)} \leq \operatorname{poly}(1 / \alpha) \sqrt{d_{\mathrm{TV}}\left(\mathcal{N}^{*}, \widetilde{\mathcal{N}}\right)}
$$

Combining the bounds for $A, B$ and $C$ we obtain the result.

Since we have the means two make both errors of Lemma 25 small we can now recover the unknown truncation set $S$.

\section{Proof of Theorem 6}

We first run Algorithm 1 to find estimates $\widetilde{\mu}, \widetilde{\Sigma}$. From Theorem 2 we know that $N=d^{\text {poly }(1 / \alpha) \Gamma^{2}(\mathcal{S}) / \varepsilon^{32}}$ samples suffice to obtain parameters $\widetilde{\mu}, \widetilde{\Sigma}$ such that $d_{\mathrm{TV}}\left(\mathcal{N}\left(\boldsymbol{\mu}^{*}, \boldsymbol{\Sigma}^{*}\right), \mathcal{N}(\widetilde{\boldsymbol{\mu}}, \widetilde{\Sigma})\right) \leq \operatorname{poly}(\alpha) \mathcal{\varepsilon}^{4}$. Notice, that from Theorem 3 we also know that $N$ samples from the conditional distribution $\mathcal{N}_{S}^{*}$ suffice to learn a function $\psi_{k}$ such that $\mathbb{E}_{\boldsymbol{x} \sim \mathcal{N}_{0}}\left[\left(\psi_{k}(\boldsymbol{x})-\psi(\boldsymbol{x})\right)^{2}\right] \leq \operatorname{poly}(\alpha) \varepsilon^{4}$. Now we can construct the approximation $\widetilde{f}(x)=\psi_{k}(x) \mathcal{N}_{0}(x) / \widetilde{\mathcal{N}}(x)$. Let our indicator $\widetilde{S}=\mathbf{1}\{\widetilde{f}((x)>1 / 2\}$ and from Lemma 24 and Lemma 25 we obtain the result.

Lemma 26. Let $P, Q$ be two distributions on $\mathbb{R}^{d}$ such that $P(\boldsymbol{x}), Q(\boldsymbol{x})>0$ for all $\boldsymbol{x}$ and $\ell: \mathbb{R}^{d} \mapsto \mathbb{R}$ be a function. Then it holds

$$
|\underset{x \sim P}{\mathbb{E}}[\ell(x)]-\underset{x \sim Q}{\mathbb{E}}[\ell(x)]| \leq\left(\underset{x \sim P}{\mathbb{E}}\left[\ell^{2}(x)\right] \underset{x \sim P}{\mathbb{E}}\right)^{1 / 2}\left(\left[\left(\frac{Q(x)}{P(x)}\right)^{2}\right]\right)^{1 / 2}
$$

Moreover,

$$
|\underset{x \sim P}{\mathbb{E}}[\ell(x)]-\underset{x \sim Q}{\mathbb{E}}[\ell(x)]| \leq 2\left(\left(\underset{x \sim P}{\mathbb{E}}\left[\ell^{2}(x)\right]+\underset{x \sim Q}{\mathbb{E}}\left[\ell^{2}(x)\right]\right)\right)^{1 / 2} \sqrt{d_{\mathrm{TV}}(P, Q)}
$$


Proof. Write

$$
\begin{aligned}
|\underset{x \sim P}{\mathbb{E}}[\ell(\boldsymbol{x})]-\underset{x \sim Q}{\mathbb{E}}[\ell(\boldsymbol{x})]| & \leq \int \ell(\boldsymbol{x}) \sqrt{P(\boldsymbol{x})} \frac{|P(\boldsymbol{x})-Q(\boldsymbol{x})|}{\sqrt{P(\boldsymbol{x})}} \mathrm{d} x \\
& =\left(\int \ell^{2}(\boldsymbol{x}) P(\boldsymbol{x}) \mathrm{d} x \int \frac{(P(\boldsymbol{x})-Q(\boldsymbol{x}))^{2}}{P(\boldsymbol{x})} \mathrm{d} x\right)^{1 / 2}
\end{aligned}
$$

For the second inequality we have

$$
\begin{aligned}
|\underset{x \sim P}{\mathbb{E}}[\ell(\boldsymbol{x})]-\underset{x \sim Q}{\mathbb{E}}[\ell(\boldsymbol{x})]| & \leq \int \ell(\boldsymbol{x})|P(\boldsymbol{x})-Q(\boldsymbol{x})| \mathrm{d} x \\
& \leq \int \ell(\boldsymbol{x}) \sqrt{P(\boldsymbol{x})+Q(\boldsymbol{x})} \frac{|P(\boldsymbol{x})-Q(\boldsymbol{x})|}{\sqrt{P(\boldsymbol{x})+Q(\boldsymbol{x})}} \mathrm{d} x \\
& \leq\left(\underset{x \sim P}{\mathbb{E}}\left[\ell^{2}(x)\right]+\underset{x \sim Q}{\mathbb{E}}\left[\ell^{2}(x)\right]\right)^{1 / 2}\left(\int \frac{(P(\boldsymbol{x})-Q(\boldsymbol{x}))^{2}}{P(\boldsymbol{x})+Q(\boldsymbol{x})} \mathrm{d} x\right)^{1 / 2}
\end{aligned}
$$

Now observe that

$$
\left(\int \frac{(P(\boldsymbol{x})-Q(\boldsymbol{x}))^{2}}{P(\boldsymbol{x})+Q(\boldsymbol{x})} \mathrm{d} x\right)^{1 / 2} \leq\left(2 \int(\sqrt{P(\boldsymbol{x})}-\sqrt{Q(\boldsymbol{x})})^{2} \mathrm{~d} x\right)^{1 / 2}=2 d_{\mathrm{H}}(P, Q) \leq 2 \sqrt{d_{\mathrm{TV}}(P, Q)}
$$

\section{F Missing Proofs of Section 6}

In the following we use the polynomial norms. Let $p(x)=\sum_{V:|V| \leq k} c_{V} x^{V}$ be a multivariate polynomial. We define the $\|p\|_{\infty}=\max _{V:|V| \leq k}\left|c_{V}\right|,\|p\|_{1}=\sum_{V:|V| \leq k}\left|c_{V}\right|$.

\section{Proof of Lemma 15}

Let $W=S_{1} \cap S_{2} \cap\left\{f_{1}>f_{2}\right\} \cup S_{1} \backslash S_{2}$, that is the set of points where the first density is larger than the second. We now write the $L_{1}$ distance between $f_{1}, f_{2}$ as

$$
\int\left|f_{1}(\boldsymbol{x})-f_{2}(\boldsymbol{x})\right| \mathrm{d} \boldsymbol{x}=\int \mathbf{1}_{W}(\boldsymbol{x})\left(f_{1}(\boldsymbol{x})-f_{2}(\boldsymbol{x})\right) \mathrm{d} \boldsymbol{x}
$$

Denote $p(x)$ the polynomial that will do the approximation of the $L_{1}$ distance. From Lemma 14 we know that there exists a Normal distribution within small chi-squared divergence of both 
$\mathcal{N}\left(\boldsymbol{\mu}_{1}, \boldsymbol{\Sigma}_{1}\right)$ and $\mathcal{N}\left(\boldsymbol{\mu}_{2}, \boldsymbol{\Sigma}_{2}\right)$. Call the density function of this distribution $g(\boldsymbol{x})$. We have

$$
\begin{aligned}
\left|\int\right| f_{1}(\boldsymbol{x}) & -f_{2}(\boldsymbol{x})\left|\mathrm{d} \boldsymbol{x}-\int p(\boldsymbol{x})\left(f_{1}(\boldsymbol{x})-f_{2}(\boldsymbol{x})\right)\right| \\
& =\left|\int\left(\mathbf{1}_{W}(\boldsymbol{x})-p(\boldsymbol{x})\right)\left(f_{1}(\boldsymbol{x})-f_{2}(\boldsymbol{x})\right) \mathrm{d} \boldsymbol{x}\right| \\
& \leq \int\left|\mathbf{1}_{W}(\boldsymbol{x})-p(\boldsymbol{x})\right|\left|f_{1}(\boldsymbol{x})-f_{2}(\boldsymbol{x})\right| \mathrm{d} \boldsymbol{x} \\
& \leq \int\left|\mathbf{1}_{W}(\boldsymbol{x})-p(\boldsymbol{x})\right| \sqrt{g(\boldsymbol{x})} \frac{\left|f_{1}(\boldsymbol{x})-f_{2}(\boldsymbol{x})\right|}{\sqrt{g(\boldsymbol{x})}} \mathrm{d} x \\
& \leq \sqrt{\int\left(\mathbf{1}_{W}(\boldsymbol{x})-p(\boldsymbol{x})\right)^{2} g(\boldsymbol{x}) \mathrm{d} x} \sqrt{\int \frac{\left(f_{1}(\boldsymbol{x})-f_{2}(\boldsymbol{x})\right)^{2}}{g(\boldsymbol{x})} \mathrm{d} \boldsymbol{x},}
\end{aligned}
$$

where we use Schwarzs' inequality. From Lemma 14 we know that

$$
\int \frac{f_{1}(\boldsymbol{x})^{2}}{g(\boldsymbol{x})} \mathrm{d} x \leq \int \frac{\mathcal{N}\left(\boldsymbol{\mu}_{1}, \boldsymbol{\Sigma}_{1} ; \boldsymbol{x}\right)^{2}}{g(\boldsymbol{x})} \mathrm{d} x=\exp (\operatorname{poly}(1 / \alpha))
$$

Similarly, $\int \frac{f_{2}(x)^{2}}{g(x)} \mathrm{d} x=\exp (\operatorname{poly}(1 / \alpha))$. Therefore we have,

$$
\left|\int\right| f_{1}(\boldsymbol{x})-f_{2}(\boldsymbol{x})\left|\mathrm{d} \boldsymbol{x}-\int p(\boldsymbol{x})\left(f_{1}(\boldsymbol{x})-f_{2}(\boldsymbol{x})\right)\right| \leq \exp (\operatorname{poly}(1 / \alpha)) \sqrt{\int\left(\mathbf{1}_{W}(\boldsymbol{x})-p(\boldsymbol{x})\right)^{2} g(\boldsymbol{x}) \mathrm{d} \boldsymbol{x}}
$$

Recall that $g(x)$ is the density function of a Gaussian distribution, and let $\mu, \boldsymbol{\Sigma}$ be the parameters of this Gaussian. Notice that it remains to show that there exists a good approximating polynomial $p(x)$ to the indicator function $\mathbf{1}_{W}$. We can now transform the space so that $g(x)$ becomes the standard normal. Notice that this is an affine transformation that also transforms the set $W$; call the transformed set $W^{t}$. We now argue that the Gaussian surface area of the transformed set $W^{t}$ at most a constant multiple of the Gaussian surface area of the original set $W$. Let $\mathcal{N}\left(\boldsymbol{\mu}_{i}, \boldsymbol{\Sigma}_{i} ; S_{i}\right)=\alpha_{i}$ for $i=1,2$ and let $h_{1}(\boldsymbol{x})=\mathcal{N}\left(\boldsymbol{\mu}_{1}, \boldsymbol{\Sigma}_{1} ; \boldsymbol{x}\right) / \alpha_{1}$ resp. $h_{2}(\boldsymbol{x})=\mathcal{N}\left(\boldsymbol{\mu}_{2}, \boldsymbol{\Sigma}_{2} ; \boldsymbol{x}\right) / \alpha_{2}$ be the density of first resp. second Normal ignoring the truncation sets $S_{1}, S_{2}$. Notice that instead of $f_{1}, f_{2}$ we may use $h_{1}, h_{2}$ in the definition of $W$, that is

$$
W=\left(S_{1} \cap S_{2} \cap\left\{h_{1} \geq h_{2}\right\}\right) \cup S_{1} \backslash S_{2} .
$$

Now, since $\boldsymbol{\Sigma}^{-1 / 2}>0$ we have that the affine map $T(x)=\boldsymbol{\Sigma}^{-1 / 2}(\boldsymbol{x}-\boldsymbol{\mu})$ is a bijection. Therefore $T(A \cap B)=T(A) \cap T(B)$ and $T(A \cup B)=T(A) \cup T(B)$. Similarly to $W^{t}=T(W)$, let $S_{1}^{t}, S_{2}^{t}$, $\left\{h_{1} \geq h_{2}\right\}^{t}$ be the transformed sets. Therefore,

$$
W^{t}=\left(S_{1}^{t} \cap S_{2}^{t} \cap\left\{h_{1} \geq h_{2}\right\}^{t}\right) \cup S_{1}^{t} \backslash S_{2}^{t} .
$$

We will use some elementary properties of Gaussian surface area (see for example Fact 17 of [KOS08]). We have that for any sets $S_{1}, S_{2} \Gamma\left(S_{1} \cap S_{2}\right)$ and $\Gamma\left(S_{1} \cup S_{2}\right)$ are upper bounded from $\Gamma\left(S_{1}\right)+\Gamma\left(S_{2}\right)$. Moreover, $\Gamma\left(S_{1} \backslash S_{2}\right) \leq \Gamma\left(S_{1}\right)+\Gamma\left(S_{2}^{c}\right)=\Gamma\left(S_{1}\right)+\Gamma\left(S_{2}\right)$. From our assumptions, we know that the Gaussian surface area of the sets $S_{1}^{t}, S_{2}^{t}$ is $O(\Gamma(\mathcal{S})$. Notice now that the set $\left\{h_{1} \geq h_{2}\right\}^{t}$ is a degree 2 polynomial threshold function. Therefore, using the result of [Kan11] (see also Table 1) we obtain that $\Gamma\left(\left\{h_{1} \geq h_{2}\right\}^{t}\right)=O(1)$. Combining the above we obtain that $\Gamma\left(W^{t}\right)=O(\Gamma(\mathcal{S})$. To keep the notation simple we from now on we will by $W$ the transformed set 
$W^{t}$. Now, assuming that a good approximating polynomial $p(\boldsymbol{x})$ of degree $k$ exists with respect to $\mathcal{N}(\mathbf{0}, \boldsymbol{I})$ then $p\left(\boldsymbol{\Sigma}^{-1 / 2}(\boldsymbol{x}-\boldsymbol{\mu})\right)$ is a polynomial of degree $k$ that approximates $\mathbf{1}_{W}(\boldsymbol{x})$ with respect to $g(x)$. Since $\mathbf{1}_{W} \in L^{2}\left(\mathbb{R}^{d}, \mathcal{N}_{0}\right)$ we can approximate it using Hermite polynomials. For some $k \in \mathcal{N}$ we set $p(\boldsymbol{x})=S_{k} \mathbf{1}_{W}(x)$, that is

$$
p_{k}(\boldsymbol{x})=\sum_{V:|V| \leq k} \widehat{\mathbf{1}_{W}} H_{V}(\boldsymbol{x})
$$

Combining Lemma 5 and Lemma 19 we obtain

$$
\underset{x \sim \mathcal{N}_{0}}{\mathbb{E}_{[}}\left[\left(\mathbf{1}_{W}(\boldsymbol{x})-p_{k}(x)\right)^{2}\right]=O\left(\frac{\Gamma(\mathcal{S})}{k^{1 / 2}}\right) .
$$

Therefore,

$$
\left|\int\right| f_{1}(\boldsymbol{x})-f_{2}(\boldsymbol{x})\left|\mathrm{d} \boldsymbol{x}-\int p_{k}(\boldsymbol{x})\left(f_{1}(\boldsymbol{x})-f_{2}(\boldsymbol{x})\right)\right|=\exp (\operatorname{poly}(1 / \alpha)) \frac{\Gamma(\mathcal{S})^{1 / 2}}{k^{1 / 4}}
$$

Therefore, ignoring the dependence on the absolute constant $\alpha$, to achieve error $O(\varepsilon)$ we need degree $k=O\left(\Gamma(\mathcal{S})^{2} / \varepsilon^{4}\right)$.

To complete the proof, it remains to obtain a bound for the coefficients of the polynomial $q(\boldsymbol{x})=p_{k}\left(\boldsymbol{\Sigma}^{-1 / 2}(\boldsymbol{x}-\boldsymbol{\mu})\right)$. We use the standard notation of polynomial norms, e.g. $\|p\|_{\infty}$ is the maximum (in absolute value) coefficient, $\|p\|_{1}$ is the sum of the absolute values of all coefficients etc. From Parseval's identity we obtain that the sum of the squared weights is less than 1 so these coefficients are clearly not large. The large coefficients are those of the Hermite Polynomials. We consider first the 1 dimensional Hermite polynomial and take an even degree Hermite polynomial $H_{n}$. The explicit formula for the $k$-th degree coefficient is

$$
\frac{2^{k / 2-n / 2} \sqrt{n !}}{(n / 2-k / 2) ! k !} \leq 2^{n}
$$

see, for example, [Sze67]. Similarly, we show the same bound when the degree of the Hermite polynomial is odd. Therefore, we have that the maximum coefficient of $H_{V}(x)=\prod_{i=1}^{d} H_{i}\left(x_{i}\right)$ is at most $\prod_{i=1}^{d} 2^{v_{i}}=2^{\sum_{i=1}^{d} v_{i}}=2^{|V|}$. Using Lemma 27 we obtain that

$$
\begin{aligned}
\left\|H_{V}\left(\boldsymbol{\Sigma}^{-1 / 2}(\boldsymbol{x}-\boldsymbol{\mu})\right)\right\|_{1} & \leq\left(\begin{array}{c}
d+|V| \\
|V|
\end{array}\right) 2^{|V|}\left(\sqrt{d}\left\|\boldsymbol{\Sigma}^{-1 / 2}\right\|_{2}+\left\|\boldsymbol{\Sigma}^{-1 / 2} \boldsymbol{\mu}\right\|_{2}\right) \\
& \leq\left(\begin{array}{c}
d+|V| \\
|V|
\end{array}\right)(4 d)^{|V| / 2}\left(O\left(1 / \alpha^{2}\right)\right)^{|V|}
\end{aligned}
$$

Now we have

$$
\|q(\boldsymbol{x})\|_{\infty} \leq \sum_{V:|V| \leq k}\left|c_{V}\right|\left\|H_{V}\left(\boldsymbol{\Sigma}^{-1 / 2}(\boldsymbol{x}-\boldsymbol{\mu})\right)\right\|_{\infty} \leq\left(\begin{array}{c}
d+k \\
k
\end{array}\right)^{2}(4 d)^{k / 2}\left(O\left(1 / \alpha^{2}\right)\right)^{k},
$$

where we used the fact that since $\sum_{V}\left|c_{v}\right|^{2} \leq 1$ it holds that $\left|c_{V}\right| \leq 1$ for all $V$. To conclude the proof we notice that we can pick the degree $k$ so that

$$
\left|\int q(\boldsymbol{x})\left(f_{1}(\boldsymbol{x})-f_{2}(\boldsymbol{x})\right)\right|=\left|\sum_{V:|V| \leq k} x^{V}\left(f_{1}(\boldsymbol{x})-f_{2}(\boldsymbol{x})\right)\right| \geq \varepsilon / 2 .
$$

Since the maximum coefficient of $q(\boldsymbol{x})$ is bounded by $d^{O(k)}$ we obtain the result. 


\section{Proof of Theorem 8}

We first draw $O\left(d^{2} / \varepsilon^{2}\right)$ and compute estimates of the conditional mean $\widetilde{\mu}_{C}$ and covariance $\widetilde{\Sigma}_{C}$ that satisfy the guarantees of Lemma 17. We now transform the space so that $\widetilde{\mu}_{C}=\mathbf{0}$ and $\Sigma_{C}=I$. For simplicity we still denote $\mu$ and $\Sigma$ the parameters of the unknown Gaussian after the transformation. From Lemma 18 we have that $\left\|\boldsymbol{\Sigma}^{-1 / 2} \boldsymbol{\mu}\right\|_{2} \leq O\left(\log (1 / \alpha)^{1 / 2} / \alpha\right)$, and $\Omega\left(\alpha^{2}\right) \leq\left\|\Sigma^{1 / 2}\right\|_{2} \leq O\left(1 / \alpha^{2}\right)$. Let $\widetilde{m}_{V}$ be the empirical moments of $\mathcal{N}(\mu, \Sigma, S), \widetilde{m}_{V}=\frac{\sum_{i=1}^{N} x^{V}}{N}$. We first bound the variance of a moment $x^{V}$.

$$
\underset{x \sim \mathcal{N}(\mu, \Sigma, S)}{\operatorname{Var}}\left[x^{V}\right] \leq \underset{x \sim \mathcal{N}(\mu, \Sigma, S)}{\mathbb{E}}\left[x^{2 V}\right] \leq \frac{1}{\alpha} \underset{x \sim \mathcal{N}(\mu, \Sigma)}{\mathbb{E}}\left[x^{2 V}\right]=\frac{1}{\alpha} \underset{x \sim \mathcal{N}(0, I)}{\mathbb{E}}\left[\left(\Sigma^{1 / 2} x+\mu\right)^{2 V}\right]
$$

Following the proof of Lemma 27 we get that $\left\|\left(\Sigma^{1 / 2} x+\mu\right)^{2 V}\right\|_{\infty} \leq\left(\sqrt{d}\left\|\Sigma^{1 / 2}\right\|_{2}+\|\mu\|_{2}\right)^{|V|}$. Using Lemma 15 we know that if we set $k=\Gamma(\mathcal{S}) / \varepsilon^{4}$ then given any guess of the parameters $\widetilde{\mu}, \widetilde{\Sigma}, \widetilde{S}$ we can check whether the corresponding truncated Gaussian $\mathcal{N}(\widetilde{\mu}, \widetilde{\Sigma}, \widetilde{S})$ is in total variation distance $\varepsilon$ from the true by checking that all moments $\mathbb{E}_{x \sim \mathcal{N}(\widetilde{\mu}, \widetilde{\Sigma}, \widetilde{S})}\left[x^{V}\right]$ of the guess are close to the (estimates) of the true moments. Using the above observations and ignoring the dependence on the constant $\alpha$ we get that $\left\|\left(\Sigma^{1 / 2} x+\mu\right)^{2 V}\right\|_{\infty} \leq d^{O(k)}$. Chebyshev's inequality implies that with $d^{O(k)} / \varepsilon^{2}$ samples we can get an estimate such that with probability at least $3 / 4$ it holds $\left|\widetilde{m}_{V}-m_{V}\right| \leq \varepsilon / d^{O(k)}$. Using the standard process of repeating and taking the median estimate we amplify the success probability to $1-\delta$. Since we want all the estimates of all the moments $V$ with $|V| \leq k$ to be accurate we choose $\delta=1 / d^{O(k)}$ and by the union bound we obtain that with constant probability $\left|\widetilde{m}_{V}-m_{V}\right| \leq \varepsilon / d^{O(k)}$ for all $V$ with $|V| \leq k$. Now, for any tuple of parameters $(\widetilde{\mu}, \widetilde{\Sigma}, \widetilde{S})$ we check whether the first $d^{O(k)}$ moments of the corresponding truncated Gaussian $\mathcal{N}(\widetilde{\mu}, \widetilde{\Sigma}, \widetilde{S})$ are in distance $\varepsilon / d^{O(k)}$ of the estimates $\widetilde{m}_{V}$. If this is true for all the moments, then Lemma 15 implies that $d_{\mathrm{TV}}(\mathcal{N}(\boldsymbol{\mu}, \Sigma, S), \mathcal{N}(\widetilde{\mu}, \widetilde{\Sigma}, \widetilde{S})) \leq \varepsilon$.

\section{Proof of Lemma 14}

Without loss of generality we may assume that $N_{1}=\mathcal{N}(\mathbf{0}, \boldsymbol{I})$ and $N_{2}=\mathcal{N}(\boldsymbol{\mu}, \boldsymbol{\Lambda})$, where $\boldsymbol{\Lambda}$ is a diagonal matrix with elements $\lambda_{i}>0$. We define the normal $N=\mathcal{N}(\mathbf{0}, \boldsymbol{R})$ with $r_{i}=\max \left(1, \lambda_{i}\right)$. We have

$$
\begin{aligned}
D_{\chi^{2}}\left(N_{2} \| N\right)+1 & =\int \frac{\mathcal{N}(\boldsymbol{\mu}, \boldsymbol{\Lambda} ; \boldsymbol{x})^{2}}{\mathcal{N}(\mathbf{0}, \boldsymbol{R} ; \boldsymbol{x})} \mathrm{d} x \\
& =\frac{\sqrt{|\boldsymbol{R}|}}{(2 \pi)^{d / 2}|\boldsymbol{\Lambda}|} \exp \left(-\boldsymbol{\mu}^{T} \boldsymbol{\Lambda}^{-1} \boldsymbol{\mu}\right) \underbrace{\int \exp \left(x^{T}\left(\frac{1}{2} \boldsymbol{R}^{-1}-\boldsymbol{\Lambda}^{-1}\right)+2 \boldsymbol{\mu}^{T} \boldsymbol{\Lambda}^{-1} \boldsymbol{x}\right) \mathrm{d} \boldsymbol{x}}_{I}
\end{aligned}
$$

We have

$$
I=\prod_{i=1}^{d} \int \exp \left(x_{i}^{2}\left(\frac{1}{2 r_{i}}-\frac{1}{\lambda_{i}}\right)+2 \frac{\mu_{i}}{\lambda_{i}} x_{i}\right) \mathrm{d} x_{i}=(2 \pi)^{d / 2} \prod_{i=1}^{d} \frac{\exp \left(\frac{2 r_{i} \mu_{i}^{2}}{2 r_{i} \lambda_{i}-\lambda_{i}^{2}}\right)}{\sqrt{2 / \lambda_{i}-1 / r_{i}}}
$$


Therefore,

$$
\begin{aligned}
D_{\chi^{2}}\left(N_{2} \| N\right)+1 & \leq \prod_{i=1}^{d} \sqrt{\frac{r_{i}}{2 \lambda_{i}-\lambda_{i}^{2} / r_{i}}} \exp \left(\frac{2 r_{i} \mu_{i}^{2}}{2 r_{i} \lambda_{i}-\lambda_{i}^{2}}\right) \\
& =\exp \left(\sum_{i=1}^{d} \frac{1}{2} \log \left(\frac{r_{i}}{2 \lambda_{i}-\lambda_{i}^{2} / r_{i}}\right)+\frac{2 r_{i} \mu_{i}^{2}}{2 r_{i} \lambda_{i}-\lambda_{i}^{2}}\right)
\end{aligned}
$$

Using the fact that $r_{i}=\max \left(1, \lambda_{i}\right)$ we have

$$
\sum_{i=1}^{d} \log \left(\frac{r_{i}}{2 \lambda_{i}-\lambda_{i}^{2} / r_{i}}\right)=\sum_{i: \lambda_{i}<1} \log \left(\frac{1}{2 \lambda_{i}-\lambda_{i}^{2}}\right) \leq \sum_{i: \lambda_{i}<1}\left(\frac{1}{\lambda_{i}}-1\right)^{2} \leq\left\|\boldsymbol{\Lambda}^{-1}-\boldsymbol{I}\right\|_{F}^{2},
$$

where we used the inequality $\log \left(1 /\left(2 x-x^{2}\right)\right) \leq(1 / x-1)^{2}$ which holds for all $x \in(0,1)$. Moreover,

$$
\sum_{i=1}^{d} \frac{2 r_{i} \mu_{i}^{2}}{2 r_{i} \lambda_{i}-\lambda_{i}^{2}}=\sum_{i: \lambda \leq 1} \frac{2 \mu_{i}^{2}}{2 \lambda_{i}-\lambda_{i}^{2}}+\sum_{i: \lambda>1} \frac{2 \mu_{i}^{2}}{\lambda_{i}} \leq \sum_{i=1}^{d} \frac{2 \mu_{i}^{2}}{\lambda_{i}}=2\left\|\boldsymbol{\Lambda}^{-1 / 2} \boldsymbol{\mu}\right\|_{2}^{2},
$$

where we used the inequality $1 /\left(2 x-x^{2}\right) \leq 1 / x$ which holds for all $x \in(0,1)$. Combining the above we obtain

$$
D_{\chi^{2}}\left(N_{2} \| N\right) \leq \exp \left(\frac{1}{2}\left\|\Lambda^{-1 / 2} \boldsymbol{\mu}\right\|_{2}+2\left\|\Lambda^{-1}-I\right\|_{F}^{2}\right)
$$

Similarly, we compute

$$
\begin{aligned}
D_{\chi^{2}}\left(N_{1} \| N\right)+1 & =\prod_{i=1}^{d} \sqrt{\frac{r_{i}}{2-1 / r_{i}}}=\exp \left(\frac{1}{2} \sum_{i: \lambda_{i}>1} \log \left(\frac{\lambda_{i}}{2-1 / \lambda_{i}}\right)\right) \\
& \leq \exp \left(\frac{1}{2} \sum_{i: \lambda_{i}>1} \lambda_{i}\left(1-\frac{1}{\lambda_{i}}\right)^{2}\right) \leq \exp \left(\frac{1}{2} \max \left(\|\boldsymbol{\Lambda}\|_{2}, 1\right)\left\|\boldsymbol{\Lambda}^{-1}-\boldsymbol{I}\right\|_{F}^{2}\right)
\end{aligned}
$$

The following lemma gives a very rough bound on the maximum coefficient of multivariate polynomials of affine transformations.

Lemma 27. Let $p(\boldsymbol{x})=\sum_{V:|V| \leq k} c_{V} x^{V}$ be a multivariate polynomial of degree $k$. Let $A \in \mathbb{R}^{d \times d}, \boldsymbol{b} \in \mathbb{R}^{d}$. Let $q(\boldsymbol{x})=p(\boldsymbol{A} \boldsymbol{x}+\boldsymbol{b})$. Then $\|q\|_{\infty} \leq\|p\|_{\infty}\left(\begin{array}{c}d+k \\ k\end{array}\right)\left(\sqrt{d}\|\boldsymbol{A}\|_{2}+\|\boldsymbol{b}\|_{2}\right)^{k}$.

Proof. We have that

$$
q(\boldsymbol{x})=\sum_{V:|V| \leq k} c_{V} \prod_{i=1}^{d}\left(\sum_{j=1}^{d} A_{i j} x_{j}+b_{i}\right)^{v_{i}}
$$

Therefore,

$$
\begin{aligned}
\|q\|_{1} & \leq \sum_{V:|V| \leq k} c_{V} \prod_{i=1}^{d}\left(\sum_{j=1}^{d}\left|A_{i j}\right|+\left|b_{i}\right|\right)^{v_{i}} \leq \sum_{V:|V| \leq k} c_{V} \prod_{i=1}^{d}\left(\|\boldsymbol{A}\|_{\infty}+\|\boldsymbol{b}\|_{\infty}\right)^{v_{i}} \\
& =\sum_{V:|V| \leq k} c_{V}\left(\|\boldsymbol{A}\|_{\infty}+\|\boldsymbol{b}\|_{\infty}\right)^{|V|} \leq\|p\|_{\infty}\left(\begin{array}{c}
d+k \\
k
\end{array}\right)\left(\|\boldsymbol{A}\|_{\infty}+\|\boldsymbol{b}\|_{\infty}\right)^{k} \\
& \leq\|p\|_{\infty}\left(\begin{array}{c}
d+k \\
k
\end{array}\right)\left(\sqrt{d}\|\boldsymbol{A}\|_{2}+\|\boldsymbol{b}\|_{2}\right)^{k}
\end{aligned}
$$

